\title{
The combined prevalence of classified rare rheumatic diseases is almost double that of ankylosing spondylitis
}

\author{
Judith Leyens ${ }^{1,2}$, Tim Th. A. Bender ${ }^{1,3}$, Martin Mücke ${ }^{1}$, Christiane Stieber ${ }^{4}$, Dmitrij Kravchenko ${ }^{1,5}$, \\ Christian Dernbach ${ }^{6}$ and Matthias F. Seide ${ }^{7^{*}}$ (D)
}

\begin{abstract}
Background: Rare diseases (RDs) affect less than 5/10,000 people in Europe and fewer than 200,000 individuals in the United States. In rheumatology, RDs are heterogeneous and lack systemic classification. Clinical courses involve a variety of diverse symptoms, and patients may be misdiagnosed and not receive appropriate treatment. The objective of this study was to identify and classify some of the most important RDs in rheumatology. We also attempted to determine their combined prevalence to more precisely define this area of rheumatology and increase awareness of RDs in healthcare systems. We conducted a comprehensive literature search and analyzed each disease for the specified criteria, such as clinical symptoms, treatment regimens, prognoses, and point prevalences. If no epidemiological data were available, we estimated the prevalence as 1/1,000,000. The total point prevalence for all RDs in rheumatology was estimated as the sum of the individually determined prevalences.
\end{abstract}

Results: A total of 76 syndromes and diseases were identified, including vasculitis/vasculopathy $(n=15)$, arthritis/ arthropathy $(n=11)$, autoinflammatory syndromes $(n=11)$, myositis $(n=9)$, bone disorders $(n=11)$, connective tissue diseases $(n=8)$, overgrowth syndromes $(n=3)$, and others $(n=8)$. Out of the 76 diseases, $61(80 \%)$ are classified as chronic, with a remitting-relapsing course in 27 cases (35\%) upon adequate treatment. Another 34 (45\%) diseases were predominantly progressive and difficult to control. Corticosteroids are a therapeutic option in 49 (64\%) syndromes. Mortality is variable and could not be determined precisely. Epidemiological studies and prevalence data were available for 33 syndromes and diseases. For an additional eight diseases, only incidence data were accessible. The summed prevalence of all RDs was 28.8/10,000.

Conclusions: RDs in rheumatology are frequently chronic, progressive, and present variable symptoms. Treatment options are often restricted to corticosteroids, presumably because of the scarcity of randomized controlled trials. The estimated combined prevalence is significant and almost double that of ankylosing spondylitis $(18 / 10,000)$. Thus, healthcare systems should assign RDs similar importance as any other common disease in rheumatology.

Keywords: Rheumatology, Rare diseases, Epidemiology, Vasculitis, Arthritis, Myositis, Fever

*Correspondence: Matthias.Seidel@szb-chb.ch

${ }^{7}$ Department of Rheumatology, Spitalzentrum-Centre hospitalier, Biel-Bienne, Switzerland

Full list of author information is available at the end of the article

\section{Background}

Rare diseases (RDs) are a complex problem in medicine, and the definition of a RD varies around the world. The European Union (EU) defines a disease as rare when it affects less than 5 people in 10,000 living in the EU, which translates to approximately 370,500 individuals being affected. In the United States, the Rare Diseases Act of 
2002 defined a disease as rare when it affects less than 200,000 people. Patients frequently remain undiagnosed for many years, and treatment is often inefficacious. Data on prevalence, disease burden, treatment regimens, access to healthcare systems, and mortality are, to a great extent, unknown. Thus, RDs remain an unresolved challenge in modern medicine.

The classification, overall prevalence, and treatment options of rare rheumatic diseases are poorly defined. Disorders may affect the musculoskeletal apparatus with arthritis and myalgia but also involve other tissues in the form of myositis, vasculitis, autoimmune organ involvement, or bone diseases. Patients are often diagnosed as having a psychosomatic disorder due to missing or unrecognized somatic and/or objective findings. Patients not only have to cope with their disease burden, but are also at risk of developing additional psychiatric comorbidities. For example, patients with undiagnosed diseases in Germany have a prevalence of depressive symptoms three times higher than the average population $(22 \%$ vs. $8.1 \%)[1,2]$. On the other hand, a verified diagnosis may aid the patient in accepting their diagnosis and coping with the ensuing symptoms and challenges. However, due to the scarcity of randomized controlled trials, treatment options for RDs are often limited and remain empirical. In addition, the definitions of key symptoms often vary in clinical studies, hampering uniform analyses.

RDs in rheumatology may be analyzed systematically by prevalence, genetic background and pathogenesis, clinical involvement, treatment options, and prognosis. Prevalence data vary according to age, as well as global and ethnic background. For example, seronegative symmetrical synovitis with pitting edema (RS3PE) has a high prevalence among the elderly, with $0.09 \%$ of all individuals over the age of 50 years being affected in Japan [3], but seems to be a quite rare disorder among younger individuals. Similarly, the prevalence of adult onset Still's disease (AOSD) varies globally: 3.4-6.9/100,000 in Norway [4], $6.77 / 100,000$ in Turkey [5], and 3.9/100,000 in Japan [6]. With respect to ethnic background, the estimated worldwide prevalence of Gaucher's disease is 1-2/100,000, but in Ashkenazi-Jews the prevalence may be as high as $1 / 850$ [7].

The pathogenesis and genetic backgrounds of some RDs in rheumatology have been studied increasingly in recent years, and in some cases well elucidated. Blau syndrome was described in 2001 and is characterized by mutations in the CARD15/NOD2 gene [8] and overexpression of autoinflammatory cytokines [9]. Interestingly, mutations in CARD15/NOD2 are also associated with other diseases with inflammatory involvement, such as Crohn's disease and arthritis [10]. Familial cold urticaria
(FCU), Muckle-Wells syndrome (MWS), and neonatalonset multisystem inflammatory disease (NOMID or chronic infantile neurologic cutaneous articular syndrome [CINCA]) were originally thought to be three similar but distinct diseases. Further evidence has shown that all three syndromes are the result of mutations in the same gene, CIAS1. They are now referred to as different phenotypes of the same disorder, namely cryoporin-associated periodic syndrome (CAPS) [11, 12]. The MEFV gene, best known for causing familial Mediterranean fever (FMF) equally demonstrates the importance of genetics in RDs [13, 14]. Heterozygous mutations in $M E F V$ are also found in many children with periodic fever, stomatitis, pharyngitis, adenitis (PFAPA) $[15,16]$. Findings suggest that exon variants in $M E F V$ may also be associated with AOSD [17].

With respect to pathogenesis, infectious agents may also play a role in RDs. As some diseases follow a distinct seasonal pattern, infectious pathogenesis has been suggested for Kawasaki disease [18, 19], and IgA-vasculitis (formerly Henoch-Schönlein purpura) [20, 21].

Furthermore, some diseases are probably modulated by hormonal alterations, such as pachydermoperiostosis [22]. Although a disease-causing genetic mutation has been detected [23], males are seemingly more commonly and severely affected [24]. RDs in rheumatology involve a variety of tissues and organ systems. For example, joints are affected by pigmented villonodular synovitis [25], the skeletal system by Camurati-Engelmann disease [26], and internal organs by AOSD [27]. The skin is involved in pyogenic arthritis, pyoderma gangrenosum, acne (PAPA) syndrome [28], blood vessels in granulomatosis with polyangiitis (GPA) [29], connective tissue in systemic sclerosis [30], and muscles in inclusion body myositis (IBM) [31].

Treatment options often, but not exclusively, include corticosteroids, such as in RS3PE [32] and eosinophilicmyalgia syndrome [33]. Although research on RDs is often limited to retrospective, single center trials or case reports only, randomized controlled trials (RCTs) have been increasingly available in recent years for some conditions, such as granulomatosis with polyangiitis [34], PFAPA syndrome [35], and FMF [36].

The disease course may be classified as self-limited (e.g., Kawasaki disease), chronic with a variable remitting-relapsing course during treatment (e.g., Takayasu arteritis), and chronic with a predominantly progressive and difficult to control course (e.g., systemic sclerosis).

The prognosis for RD varies and may be affected by the primary disease, complications, and treatment, especially long-term immunosuppression. 
The objective of this study was to analyze the complexity of RDs in rheumatology. Based on data from the literature, we identified some of the most important sets of rheumatic diseases and calculated their combined prevalence. Our data may give better insight into this area of rheumatology, aid specialized centers for RDs, and raise overall awareness in the healthcare field.

\section{Results}

The diseases extracted from the databases are summarized in Table 2 of Appendix. The 76 syndromes and diseases were classified as follows: vasculitis/vasculopathy $(n=15)$, arthritis/arthropathy $(n=11)$, autoinflammatory syndromes $(n=11)$, myositis $(n=9)$, bone disorders $(n=11)$, connective tissue diseases $(n=8)$, which include inflammatory as well as non-inflammatory conditions, overgrowth syndromes $(n=3)$, and others $(n=8)$. A definitive genetic cause was identified in 26 diseases (34\%). Out of the 76 conditions, 34 diseases (44\%) were classified as chronic, primarily progressive, and difficult to control. Twenty-seven diseases (35\%) were classified as chronic with a variable remitting-relapsing course. Six diseases (7\%) were classified as self-limited. Acute phase reactants may be elevated in 49 diseases (64\%). Corticosteroids are used as a therapeutic option in 49 diseases (64\%). The mortality was variable and could not be determined precisely, but nine diseases $(11 \%)$ were considered severe and potentially lethal if left untreated.

Prevalence data were available for 28 syndromes and diseases. For an additional five diseases, estimated prevalence data were already available. For another eight diseases, only incidence data were available. The prevalence of 38 diseases was estimated at 1/1,000,000, for 4 diseases at 1/100,000, and for 1 disease at 1/10,000. The combined prevalence per 10,000 is given in Table 1 (see also Figs. 1 and 2). The summed prevalence of all available and estimated RDs was 28.8/10,000.

\section{Discussion}

RDs are challenging for patients, healthcare professionals, and societies. Signs and symptoms often remain unrecognized and patients are excluded from specific treatment. Patients are frequently diagnosed with a psychosomatic disorder. Conversely, some patients with true extrasomatic diagnoses insist on the presence of a $\mathrm{RD}$ and cause significant expenses to healthcare systems. To overcome this bias, the importance of RDs should be recognized in public healthcare.

The knowledge of RDs is mostly available from case reports or case studies. On one hand, these studies are important in order to document essential information, such as commonly reported symptoms, different treatment regimens, and outcomes. However, such studies may involve reporting bias and, thus, are difficult to compare. For example, multicentric histiocytosis is a disease primarily reported in Caucasian women. However, this may be due to increased awareness in Western countries. Furthermore, women may consult a doctor more often and simulate a female Caucasian predominance [37].

Most studies scrutinizing RDs are designed as single center, retrospective studies due to a lack of patient numbers or networks. Larger registries would provide an opportunity to conduct retrospective or prospective and multicenter studies with a greater number of participating patients. Therefore, further development of international expert centers and registries is in great demand. In recent years, advances have been made due to the establishment of international expert/reference centers. For example, the Eurofever project for autoinflammatory diseases provided classification criteria and evaluated treatment options for multiple disorders [38, 39].

In addition, genetic testing has become increasingly available and, thus, more important in recent years. For example, whole exome sequencing in patients with similar symptoms without previous knowledge of candidate genes led to the identification of WISP 3 and SLCO2A1 as the pathogenic mutations in progressive pseudorheumatoid dysplasia [40] and primary hypertrophic osteoarthropathy [23].

In this study, we aimed to identify and classify RDs in rheumatology. We were able to show that the most common symptoms in rare rheumatic diseases are arthritis $(31.0 \%, 89.3 / 100,000)$, followed by vasculitis $(26.6 \%$, $76.8 / 100,000)$, and connective tissue involvement $(16.0 \%$, $46.1 / 100,000)$, which in this study includes inflammatory as well as non-inflammatory conditions. Importantly, the total prevalence of a symptom was commonly dominated by only a few comparatively prevalent diseases. For example, systemic sclerosis (prevalence: 22.5/100,000) makes up $48.8 \%$ of all rare rheumatic diseases with connective tissue involvement.

Our study has several limitations. First, the nomenclature for diseases and syndromes is often used interchangeably, and the same disease or a variation in the same group of diseases is sometimes named differently. For example, CAPS is formerly known as Muckle-Wells, CINCA/NOMID, or familial cold autoinflammatory syndrome [12]. This may lead to incomplete search results and impede the comparability of available studies. Second, most prevalence data are almost exclusively based on retrospective analyses of hospital information or questionnaires. Prevalence data also vary according to ethnic background, geography, and age, which makes the use of overall prevalence data quite uncertain. For 


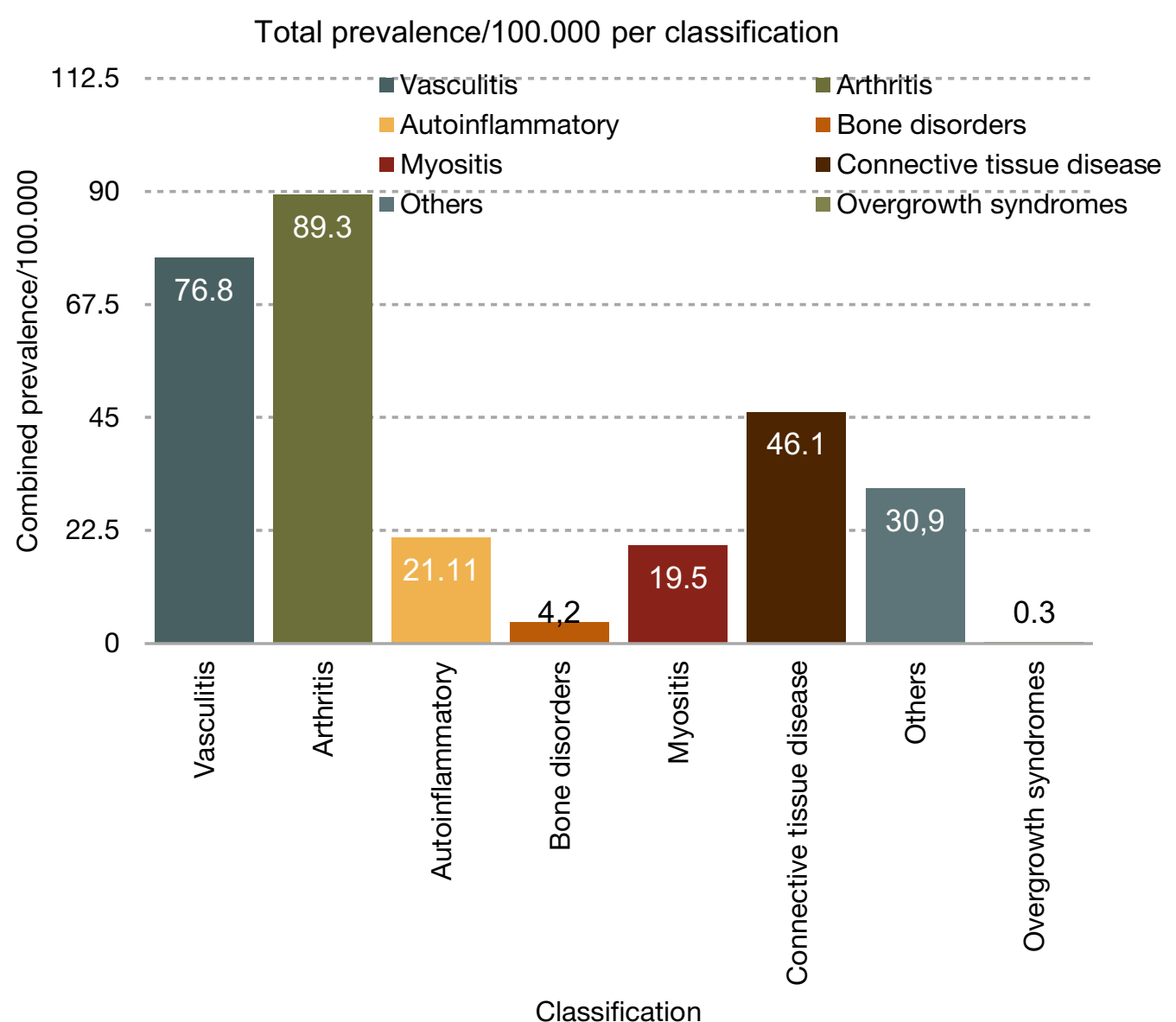

Fig. 1 Overview of the total number of diseases in each classification

example, FMF or Behçet's are more common in Mediterranean and Middle Eastern populations [41, 42] and rare in other regions, demonstrating the difficulty in using a local geographic prevalence. Similarly, although currently considered a RD by the European definition, an Italian study found an unexpectedly high overall prevalence of 8.5/10,000 for cryoglobulinemic vasculitis, which would no longer be defined as a RD. Although this study had some limitations, including the implementation of a questionnaire leading to a higher participation rate in the elderly population than the younger age groups, we decided to exclude cryoglobulinemic vasculitis from our list of rare rheumatological diseases. Our reasoning for this was the methodically more accurate estimation of prevalence by this study [43]. A well-known, verified RD, systemic sclerosis is quite common in Choctaw Native Americans (prevalence 66/100,000 in Oklahoma Choctaws and 469/100,000 in full-blooded Choctaws
[44]), but rare among all other studied populations [45]. This may be due to a unique HLA haplotype, but other environmental factors may also play a role. Furthermore, our estimates of prevalence data may be somewhat inaccurate. We probably underestimated the prevalence by choosing 1 in 1,000,000 rather than 1 in 100,000, and the total prevalence of rare rheumatic diseases is likely to be even higher than our estimate of 29.6/10,000.

Prevalence data may also differ depending on age. For example, giant cell arteritis is probably extremely rare in younger patients but quite frequent in patients older than 55 years of age (UK 250/100,000 [46]), with ageindependent prevalence data being difficult to obtain. Because the overall estimated prevalence may be even higher than 1/10,000, we also excluded giant cell arteritis from our list of RDs in rheumatology.

Similarly, we also excluded systemic lupus erythematodes, one of the better known "rare" diseases in 


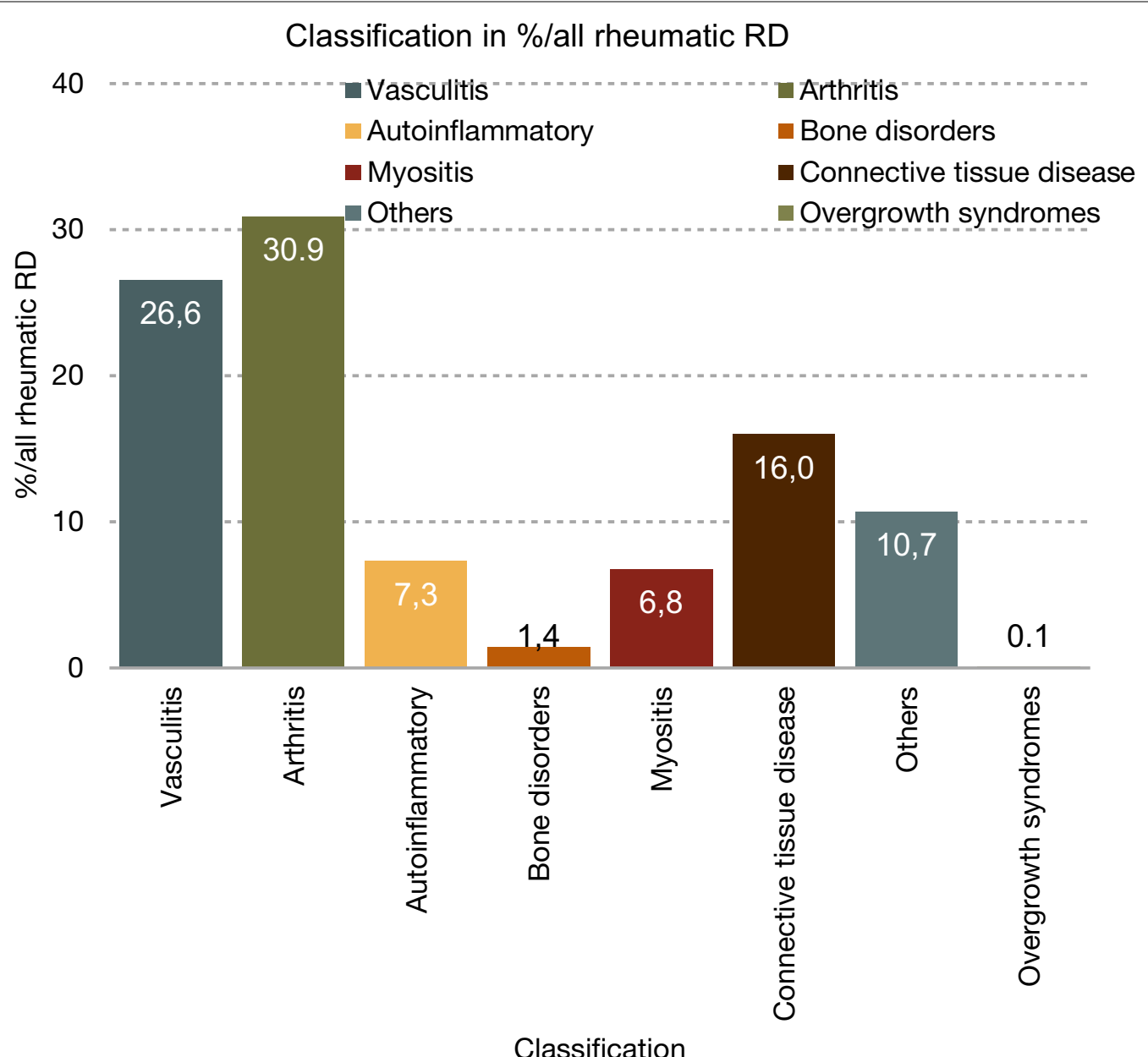

Fig. 2 Flow chart of syndrome inclusion in the analysis. After conducting the literature search and analysis with regard to prevalence, four diseases were excluded due to high prevalence, and seven diseases were merged into three, resulting in a total of 76 syndromes included in further analysis

rheumatology from our study, as recent epidemiological studies suggest that it is probably not a rare disease by the above mentioned European definition [47]. Furthermore, we also excluded rhupus syndrome, because it is suspected to be an overlap of systemic lupus erythematodes and rheumatoid arthritis and thus its prevalence may be difficult to obtain and distinguish [48].

The classification system we used also has its limitations. Conditions can be classified by their etiology or by their clinical appearance. As the etiology of many rare diseases remains unknown, we decided to classify diseases according to their main organ system involved in the clinical appearance of the disease. Exceptions include the category of autoinflammatory and overgrowth syndromes, where multiple organ systems may be involved, and the conditions in one group have a basic (suspected) etiology in common. However, overlaps between included conditions may have occurred because of the complex nature of some RDs.

Another pitfall is that diagnostic and/or classification criteria may differ in varying definitions of the diseases (e.g., familial Mediterranean fever), are not well established, and have been suggested based on radiographic or histological findings. In most cases, no validation studies are available to confirm specificity and sensitivity.

We observed that RCTs are available only for some RDs, such as Behcet's disease [49] or ANCA-associated vasculitis [34]. Furthermore, most follow-ups of patients with RDs are rarely published. Larger patient cohorts would be necessary to obtain reliable data on outcome, disease progression, morbidity, and mortality. Treatment complications in most, if not all, RDs may be due to the 


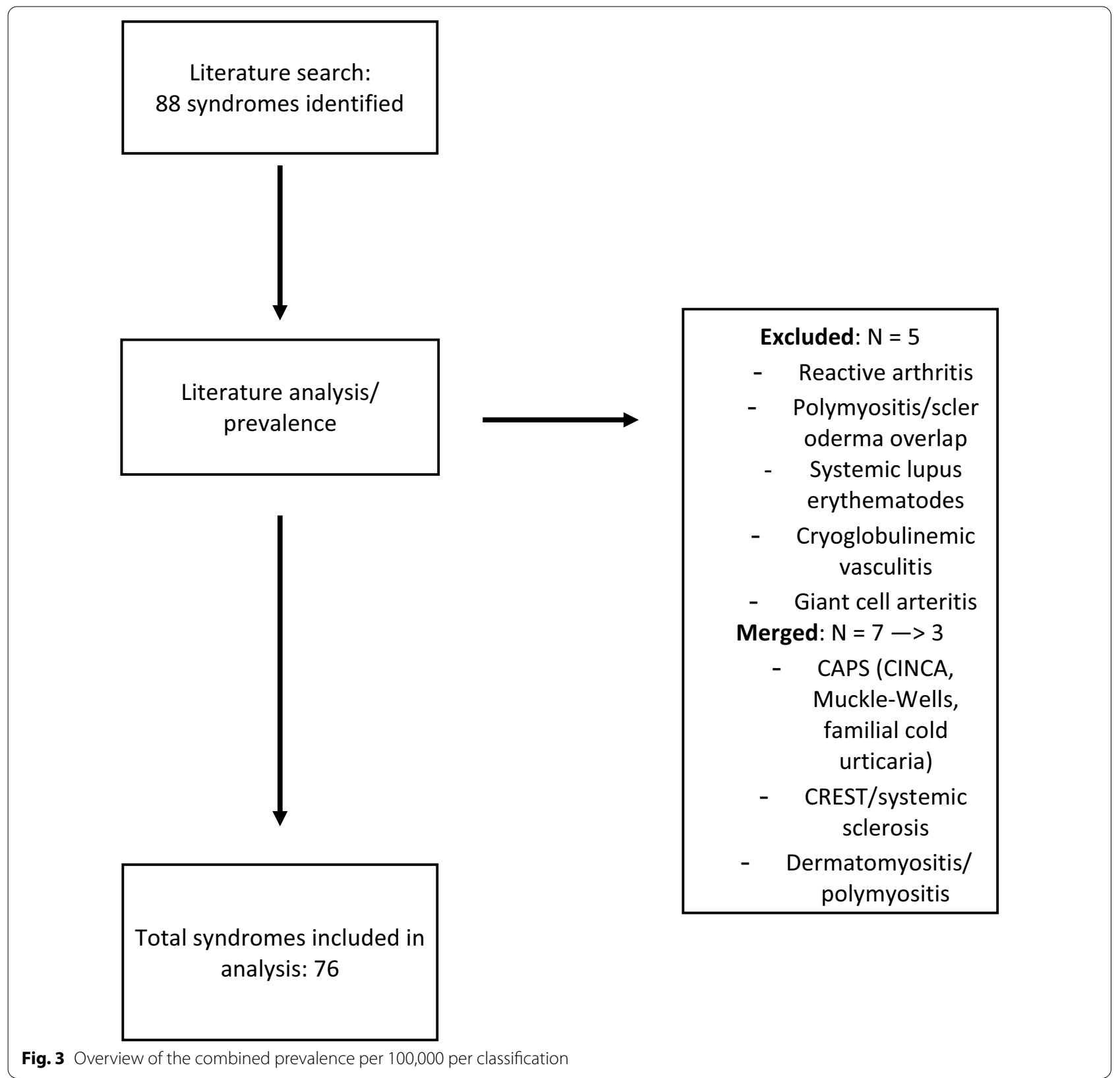

disease itself or adverse events to immunosuppressive treatment. These data merit in-depth analyses, as they may shed more light on the disease and its pathophysiology or potential treatment options.

Incidence and prevalence data have become available for increasingly more RDs and, in some cases, the prevalence has even increased in recent years (e.g., Kawasaki disease) [50]. This trend may be due to a true increase in incidence, increased diagnosis of previously undiagnosed patients due to an upsurge in physician or patient awareness (internet, patient support groups, RD associations, etc.), or simply due to better national reporting systems and databases. In addition, an increased overall life expectancy in the general population has led to an 
increased incidence and prevalence of conditions that predominantly affect the elderly (e.g., GCA). Continuously better treatment options may also lead to prolonged overall survival with an increase in prevalence rates.

In this study, we found that the cumulative prevalence of RDs in rheumatology is at least $28.8 / 10,000$, which is almost double that of ankylosing spondylitis $(18 / 10,000)$ [51], a rather common disease seen in practice. This observation suggests that symptoms should be carefully acknowledged in all patients, especially when an overt psychopathology is present, as many patients with a yet undiagnosed disorder show signs of depression or other psychosomatic disorders [1], which can obscure the differentiation between primary disease and secondary complication even more for the treating physician. Our study may aid physicians as a simple tool for diagnosing patients with an undiagnosed rheumatic disease by comparing the symptoms, prevalence, and likeliness of one disorder to another.

\section{Conclusion}

Our study shows that RDs in rheumatology are not as rare as previously thought, affecting at least $28.8 / 10,000$ people. Therefore, for every patient diagnosed with ankylosing spondylitis, 1.6 patients may suffer from a rare rheumatic disease. Although research and case reports of RDs are important, international expert centers are necessary to initiate and perpetuate patient cohorts and registries, establish classification/diagnostic criteria, and conduct clinical trials. Standard questionnaires and laboratory analyses may aid in obtaining better insight into the pathophysiology of RDs.

\section{Methods and materials}

The abstract archives of the European League Against Rheumatism, the American College of Rheumatology, Orpha.net, and the PubMed database were searched for the following terms: "rare" in combination with arthritis, arteritis, connective tissue disease, rheumatic, and vasculitis. Furthermore, terms were used in various combinations including arthralgia, autoimmune, fever, inflammation, joint pain, muscle pain, myalgia, and swollen joint. Identified syndromes were then classified according to their main appearance under the following terms: arthritis/arthropathy, bone disorders, autoinflammatory syndromes, connective tissue diseases, myositis/ myopathy, overgrowth syndromes, vasculitis/vasculopathy, and others. Furthermore, we conducted a search of the literature and analyzed each disease according to the following criteria: prevalence, genetics/pathogenesis, diagnostic criteria, symptoms, laboratory findings, therapy, and prognosis. Diseases and syndromes were assessed for their overall prevalence and excluded if they did not meet the European definition of a $\operatorname{RD}(<5 / 10,000)$ (see Fig. 3).

If more than one prevalence was available, the prevalence data were averaged accordingly.

For statistical reasons, we estimated the prevalence for diseases for which no epidemiological data were available as one of three possible values: $<1 / 1,000,000,1 / 100,000$ or $1 / 10,000$. The overall total prevalence for all RDs in rheumatology was estimated by summing up the available or estimated individual prevalence of each disease.

\section{Appendix}

See Table 1, 2.

Table 1 Results from the literature analyses

\begin{tabular}{lccl}
\hline Classification & $\mathbf{N}=\begin{array}{l}\text { Combined } \\
\text { prevalence/100.0000 }\end{array}$ & $\begin{array}{l}\text { \%/All RD } \\
\text { (prevalence) }\end{array}$ \\
\hline Vasculitis & 15 & 76.8 & 26.55 \\
Arthritis & 11 & 89.3 & 30.9 \\
Autoinflammatory & 11 & 21.105 & 7.32 \\
Bone disorders & 11 & 4.15 & 1.44 \\
Myositis & 9 & 19.5 & 6.76 \\
Connective tissue disease & 8 & 46.1 & 15.99 \\
Others & 8 & 30.89 & 10.72 \\
Overgrowth syndromes & 3 & 0.3 & 0.1 \\
Total & 76 & 288.15 & 100 \\
\hline
\end{tabular}




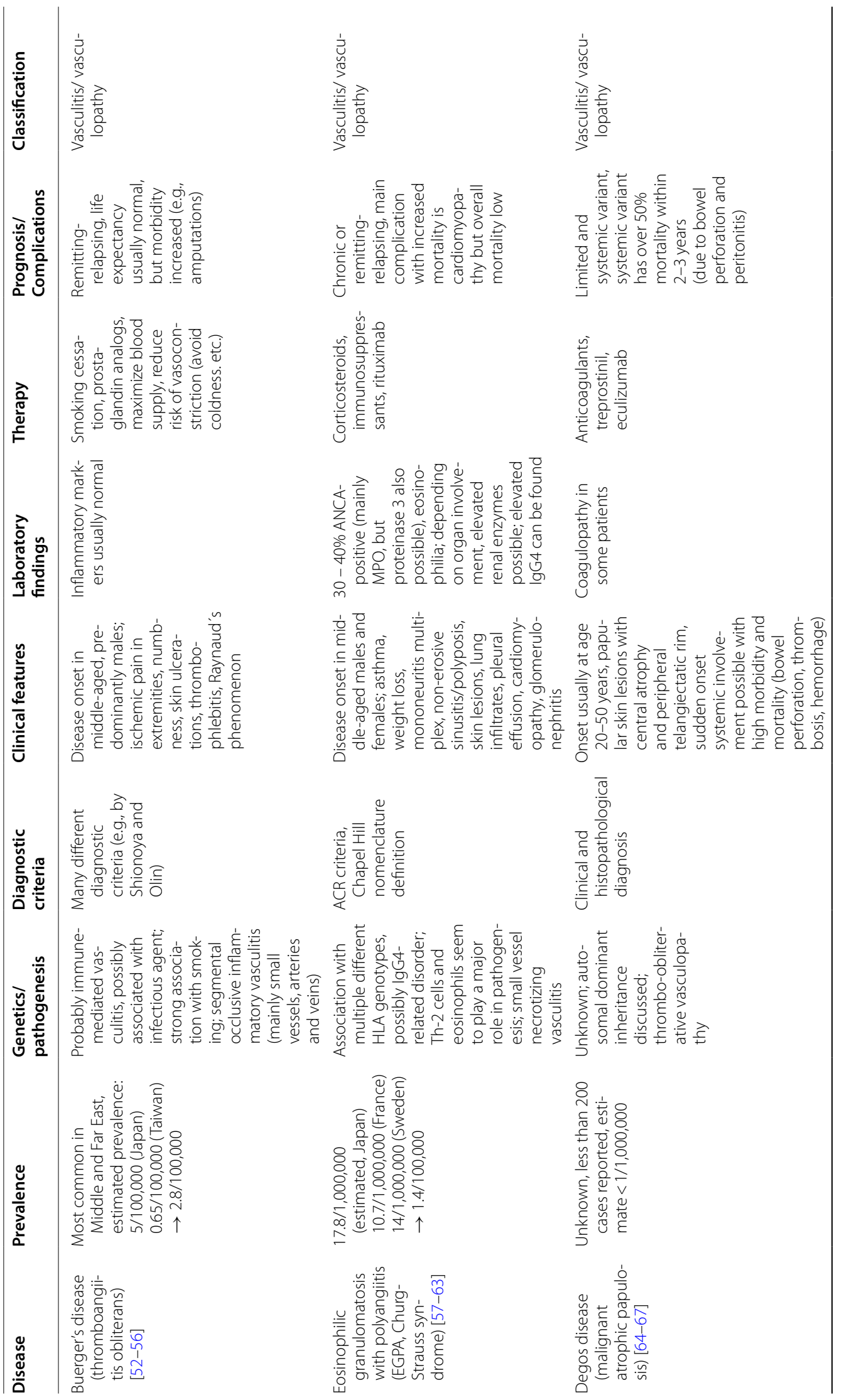




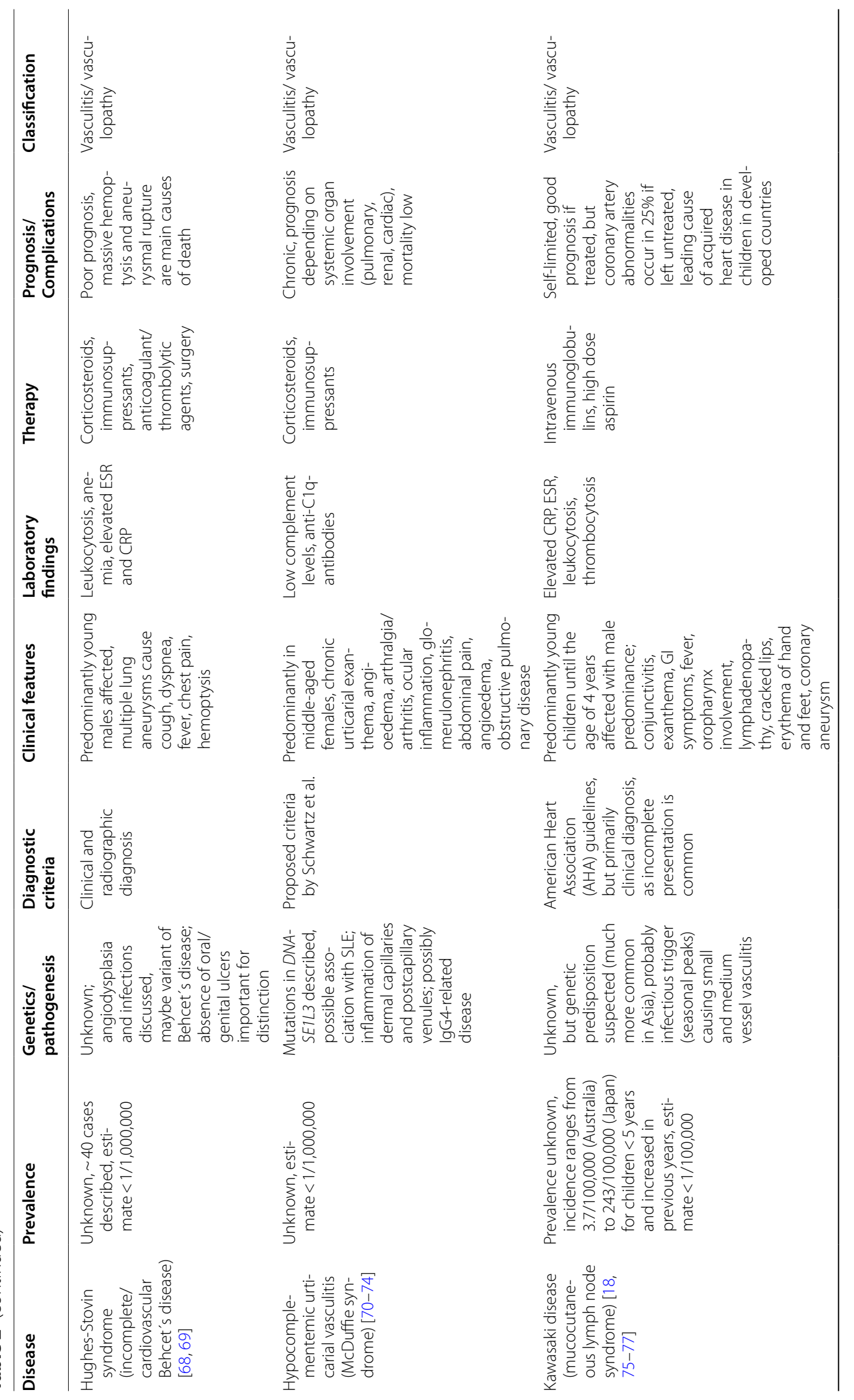




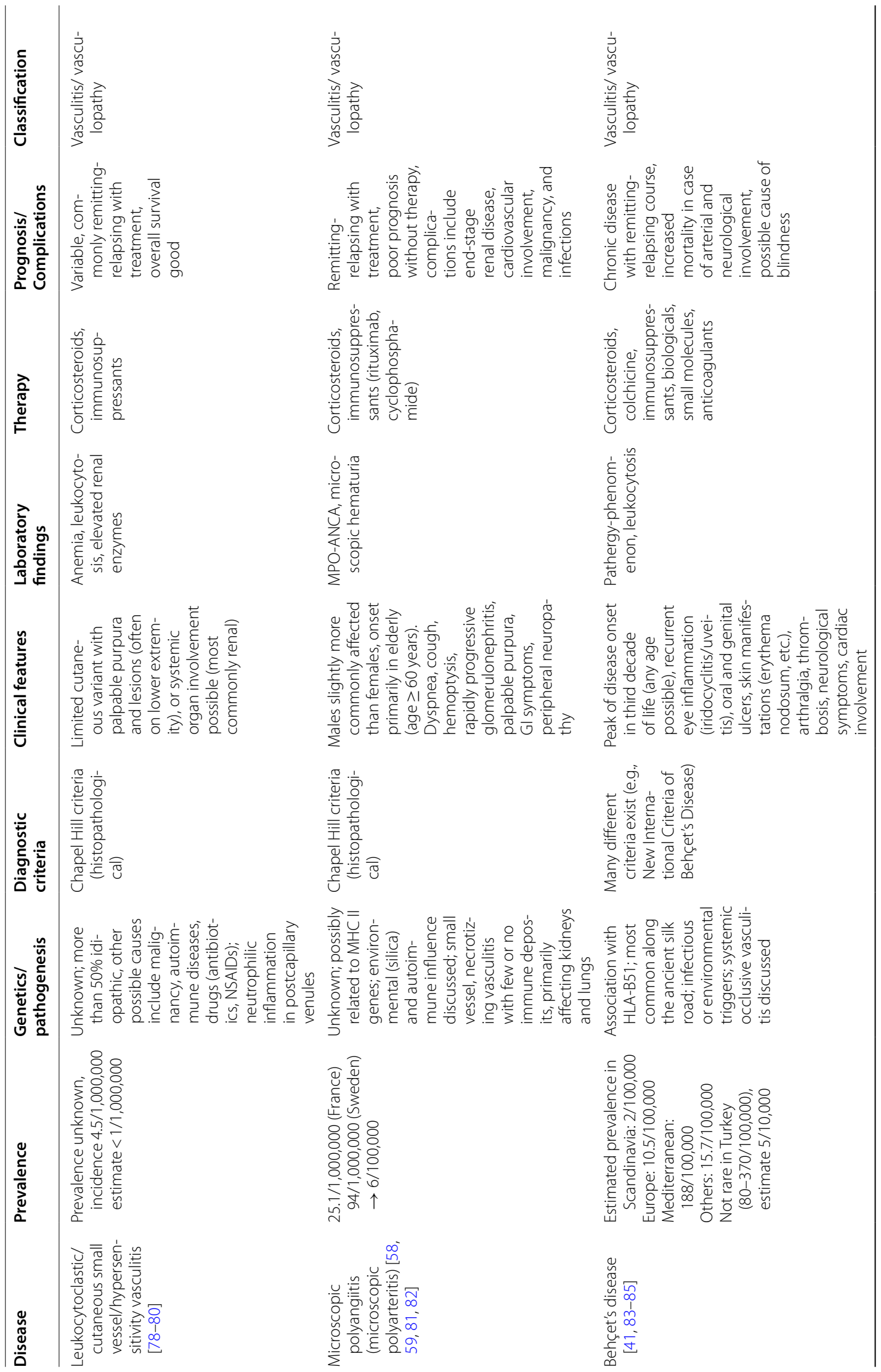




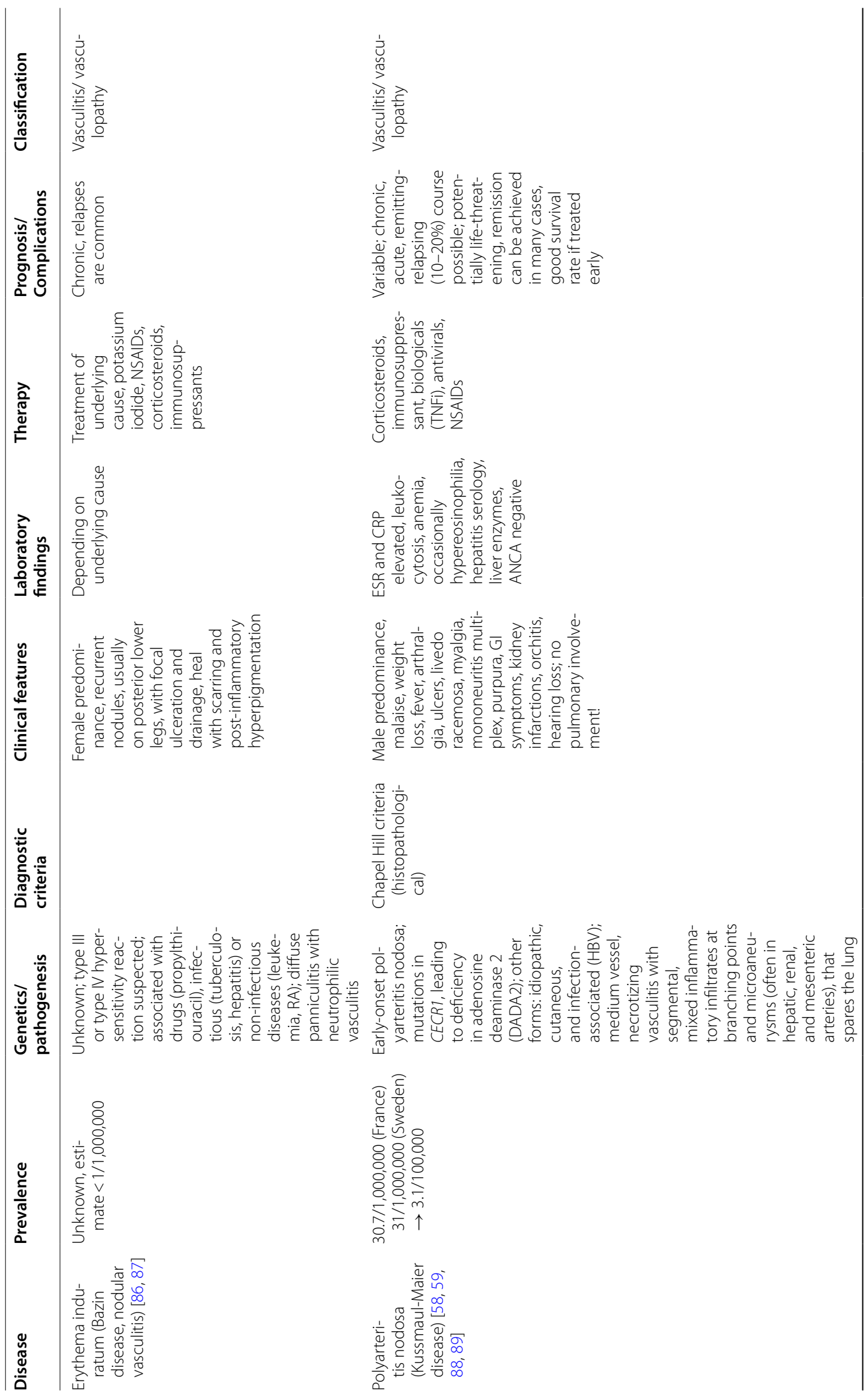




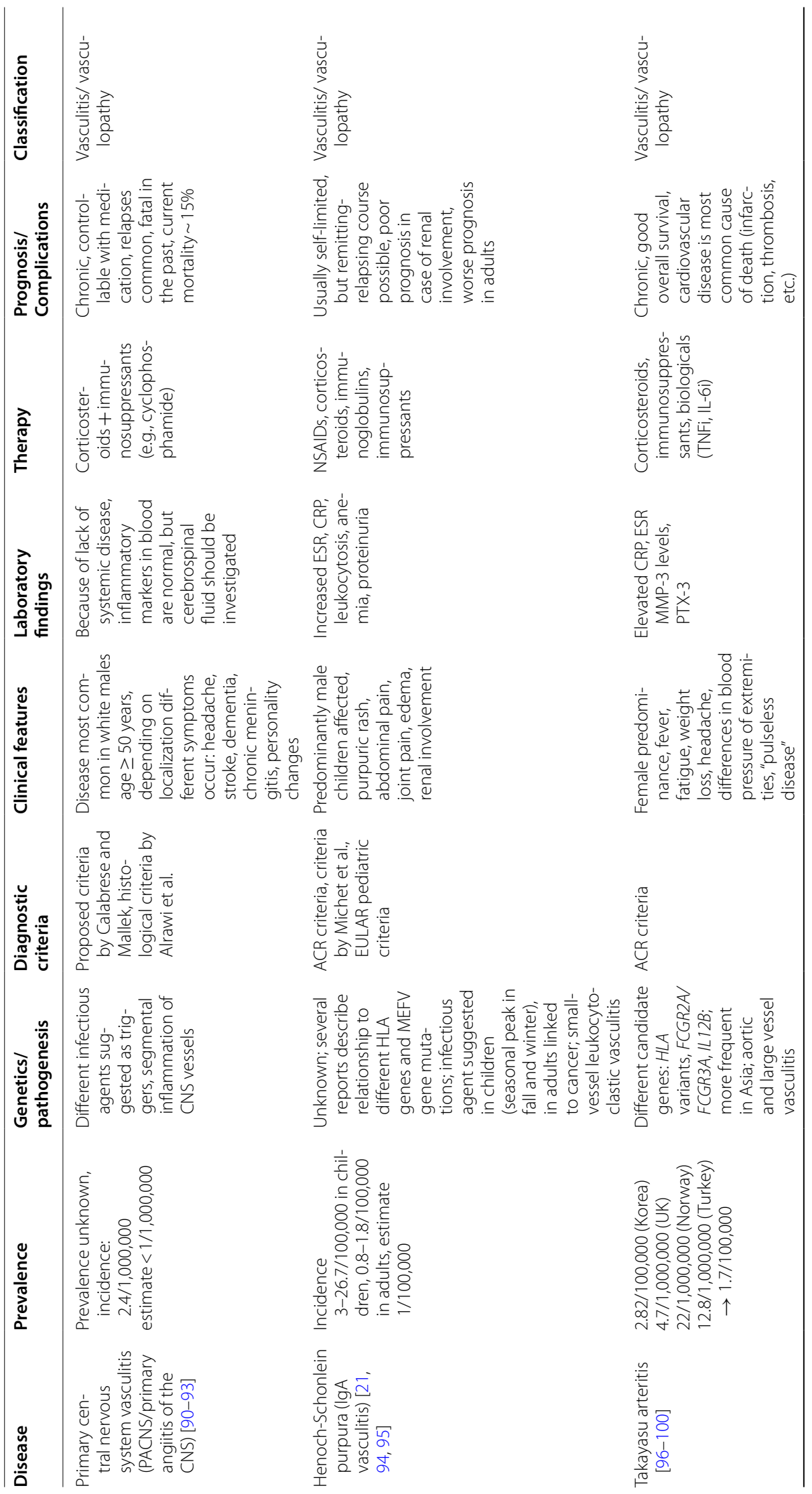




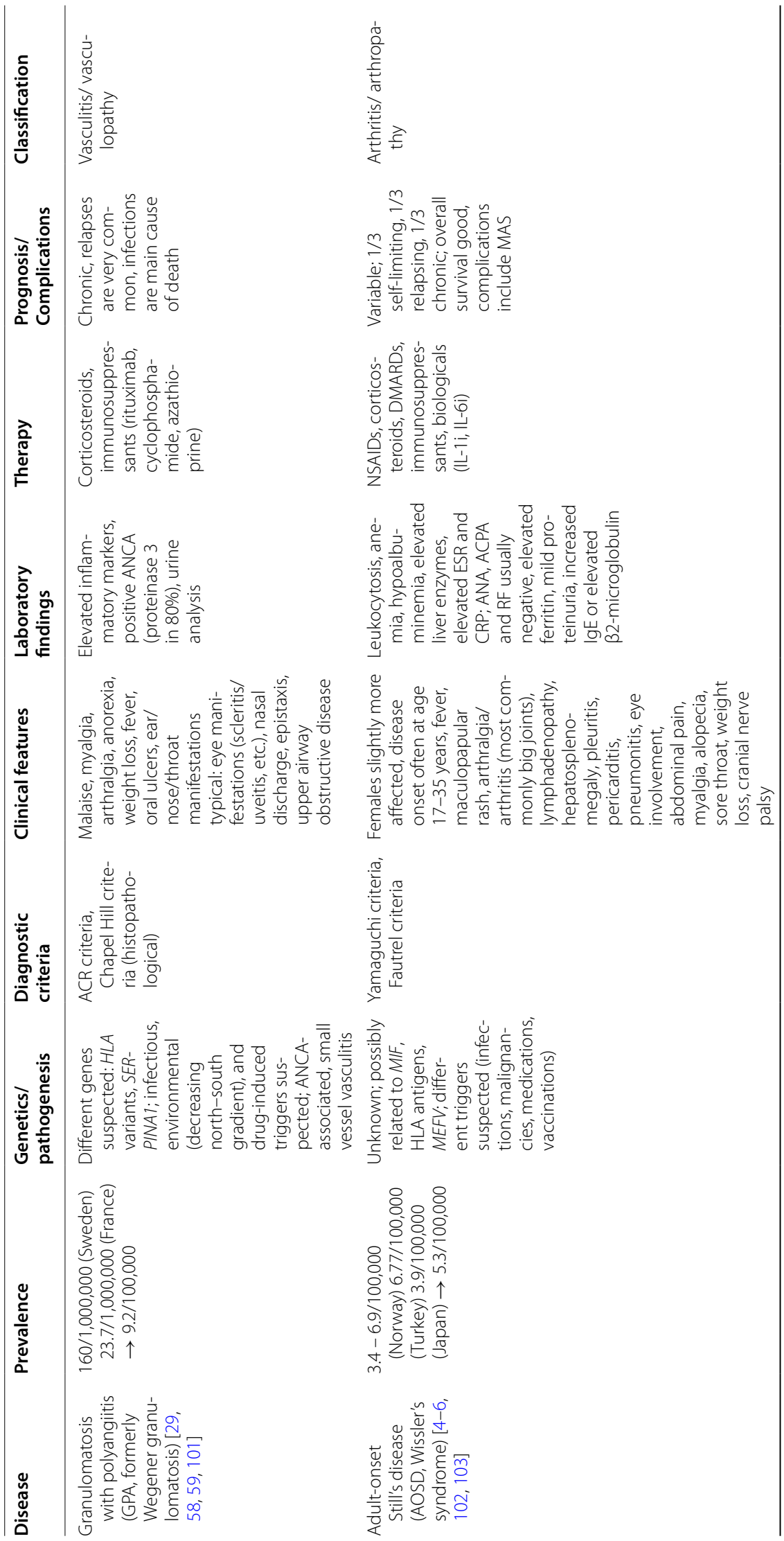




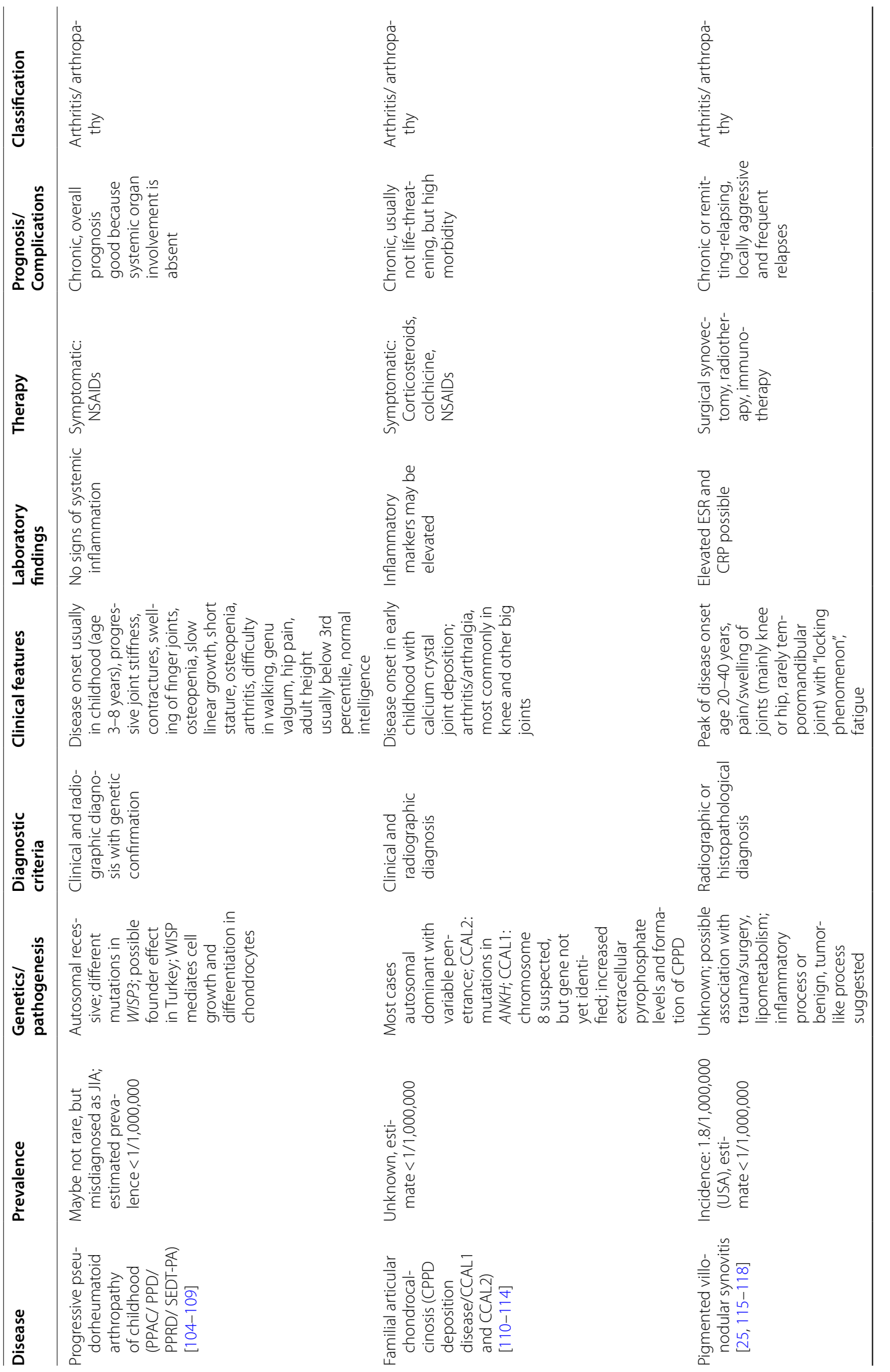




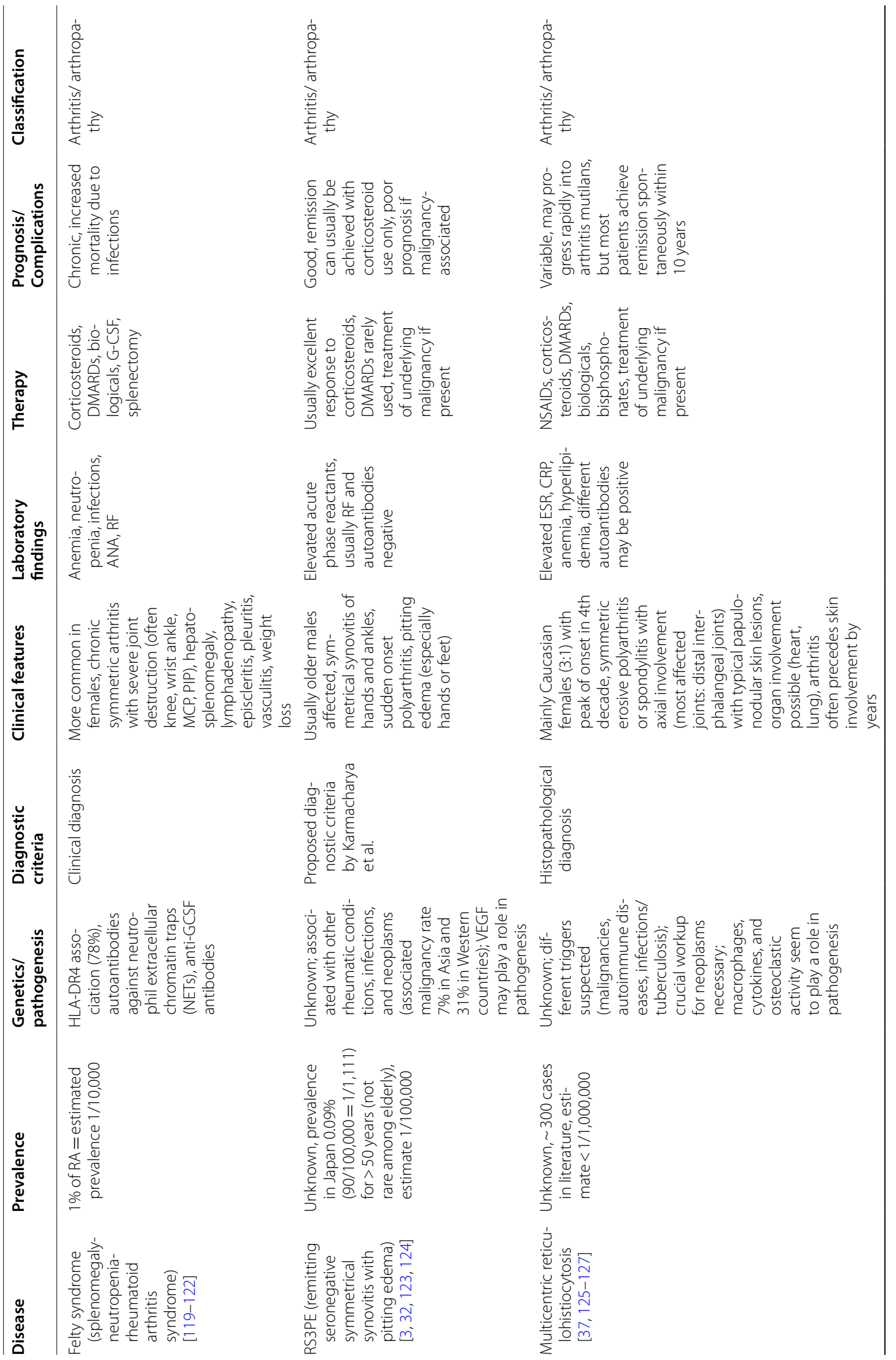




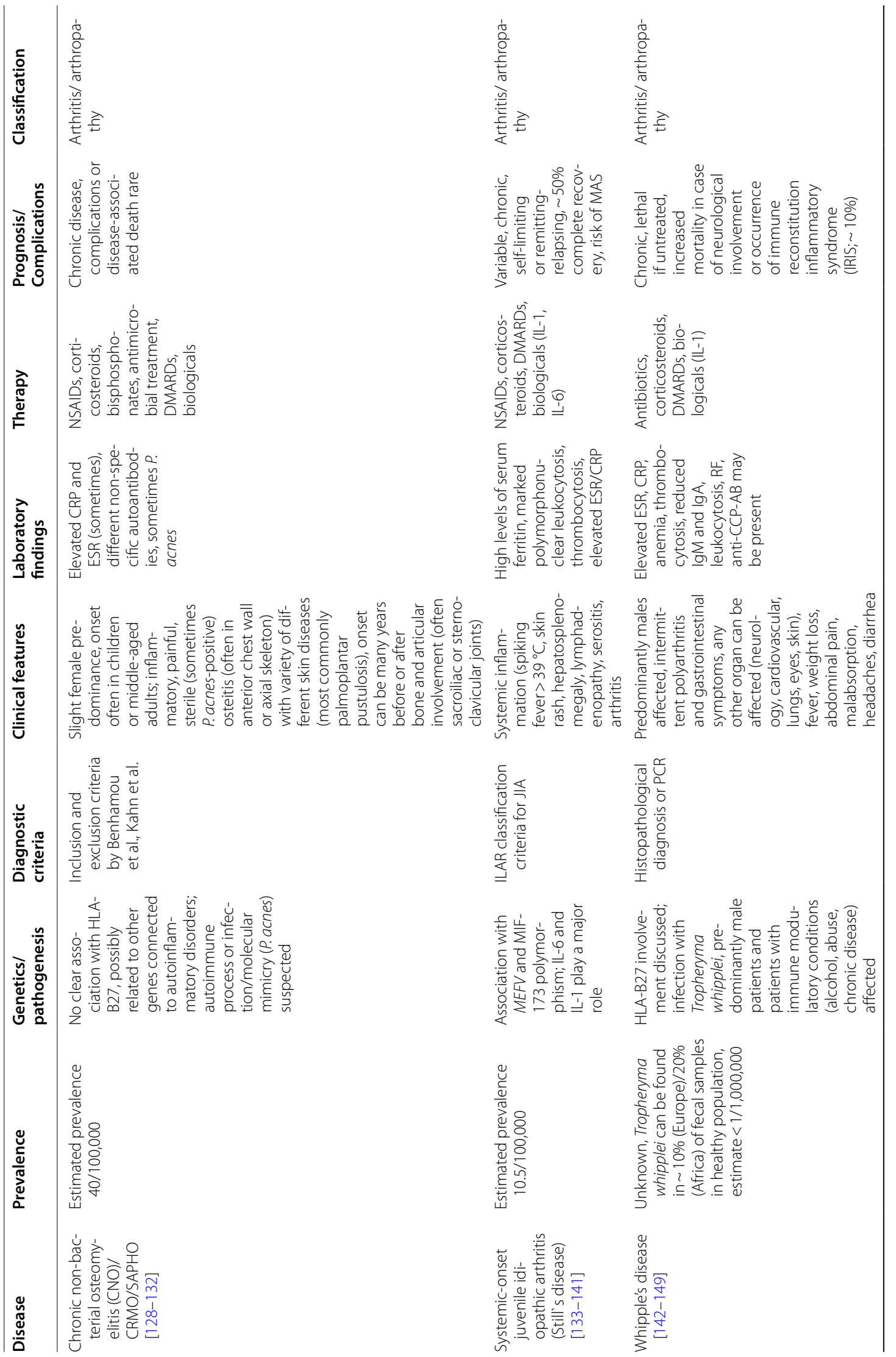




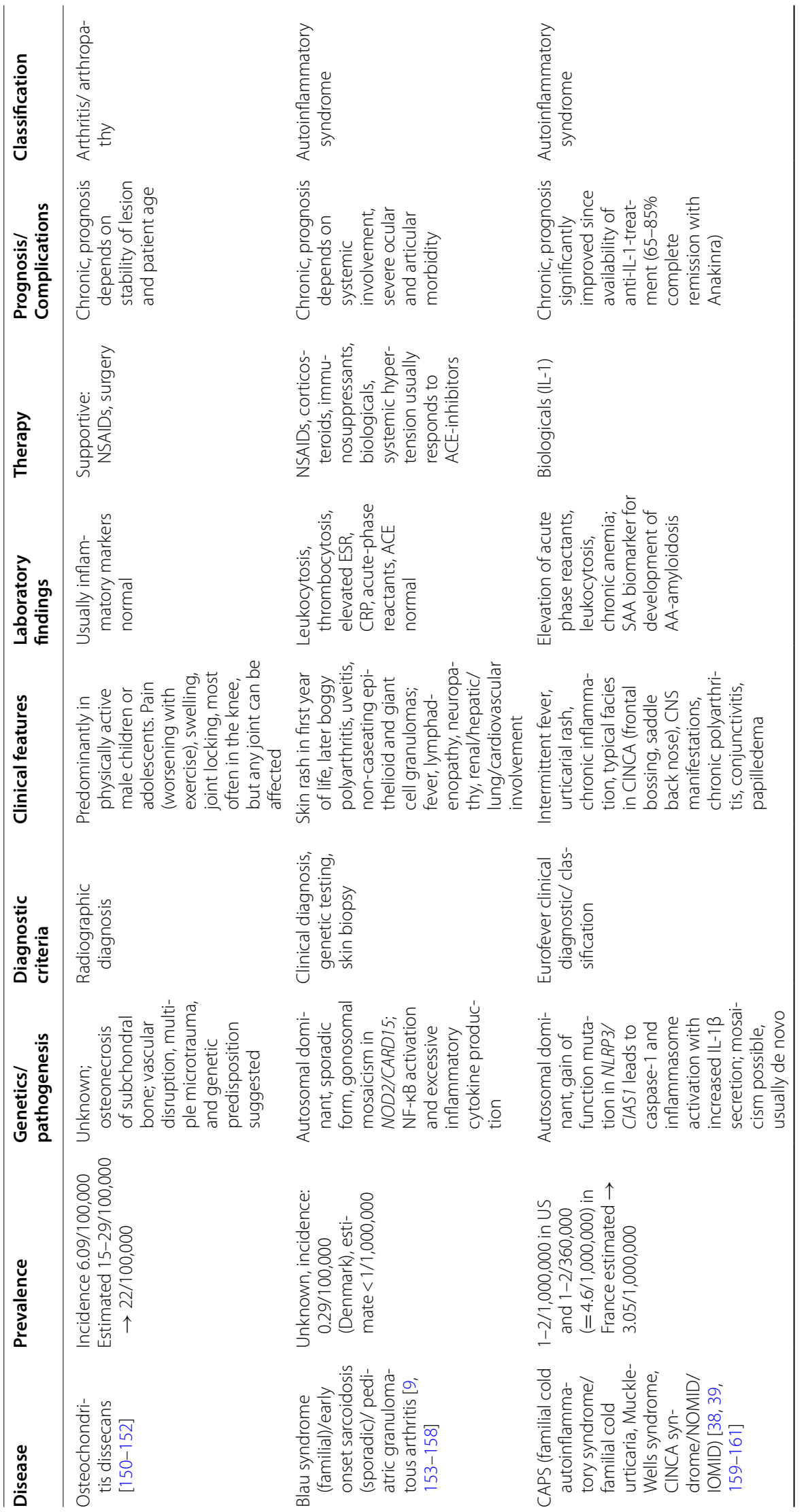




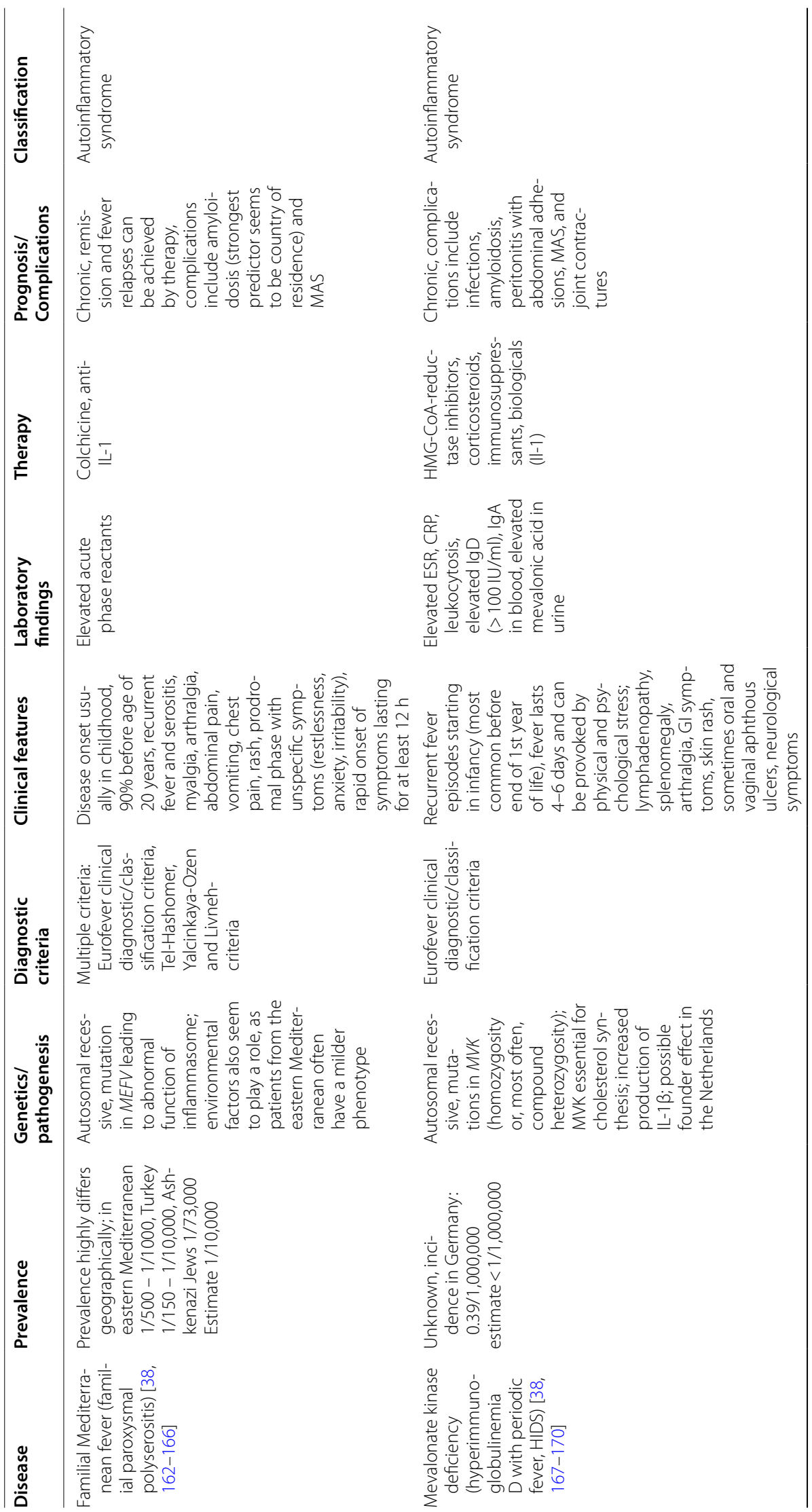




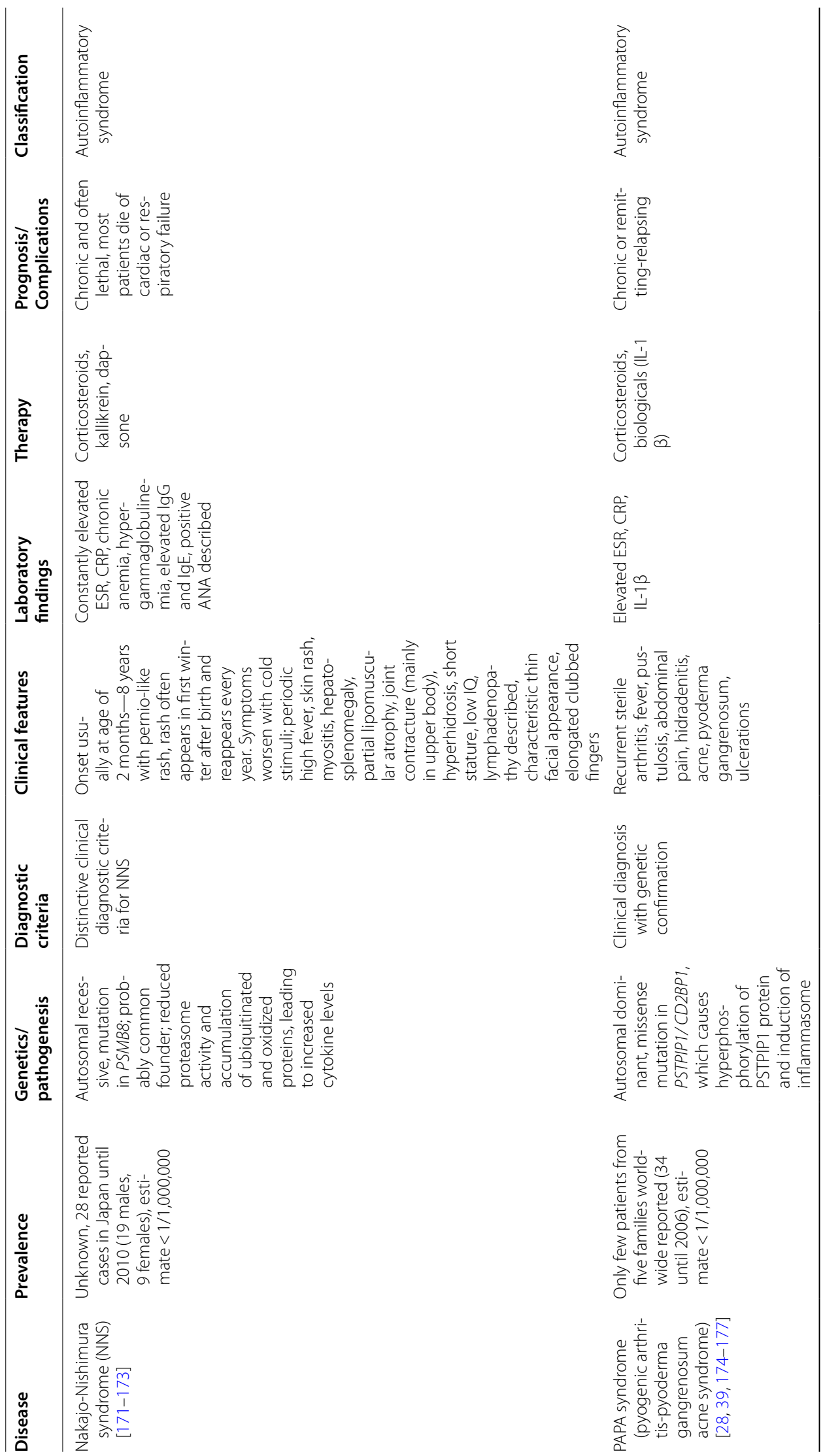




\begin{tabular}{|c|c|c|c|}
\hline & 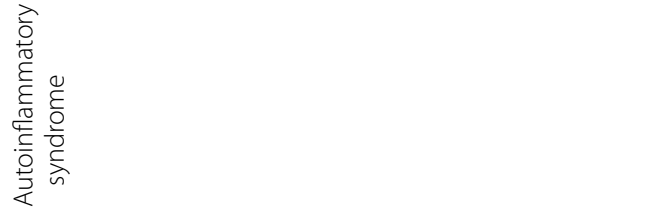 & 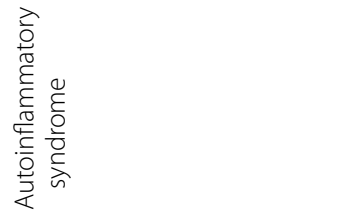 & 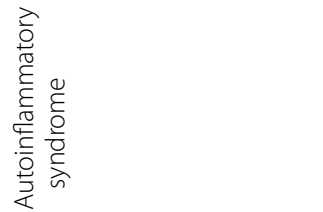 \\
\hline 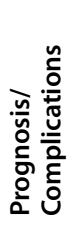 & 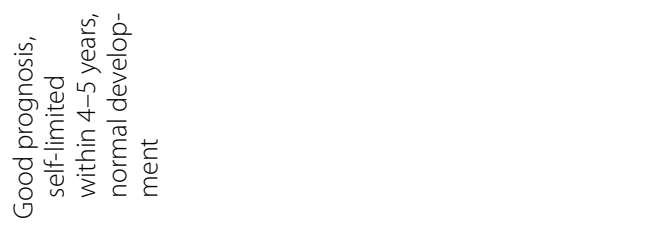 & 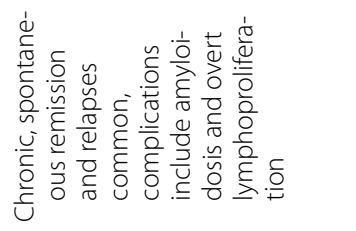 & 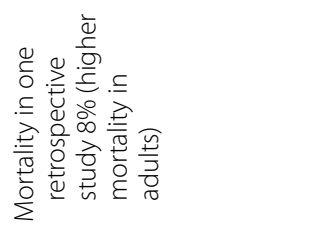 \\
\hline & 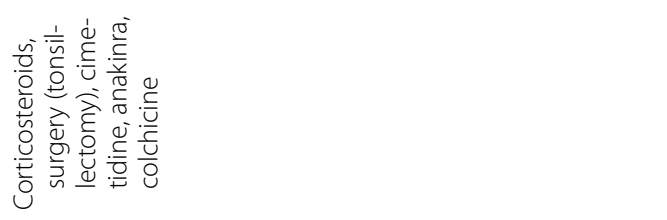 & 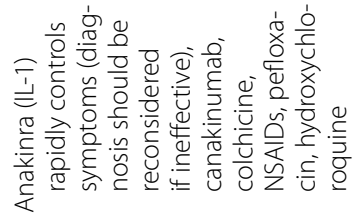 & 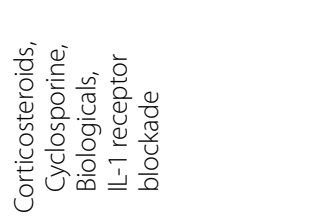 \\
\hline & 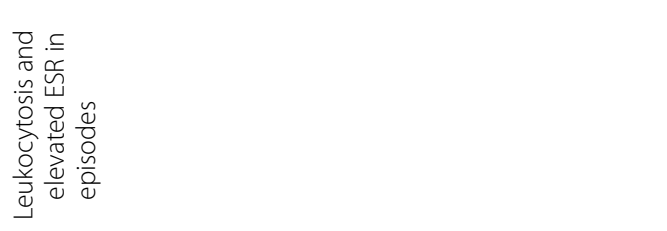 & 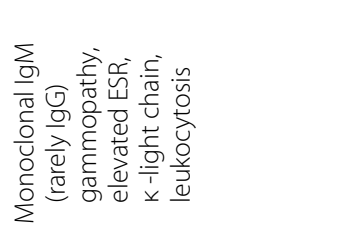 & 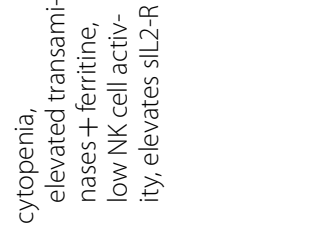 \\
\hline & 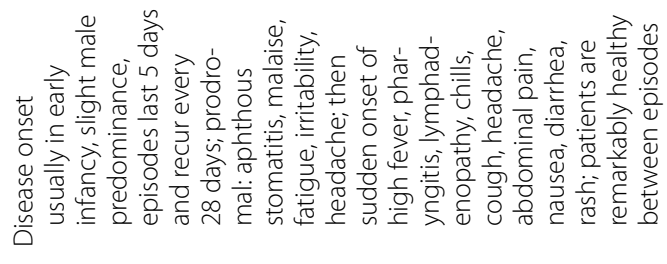 & 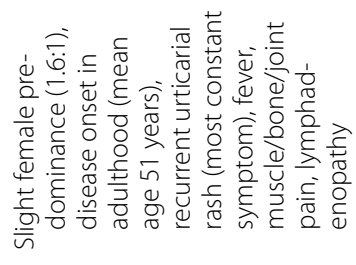 & 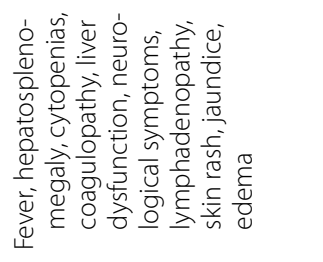 \\
\hline 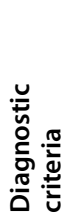 & 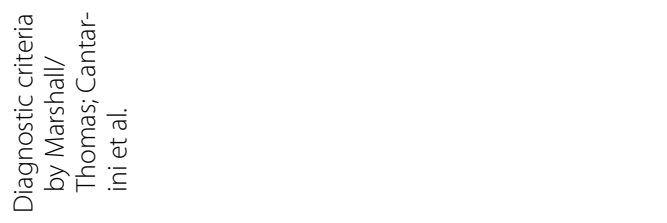 & 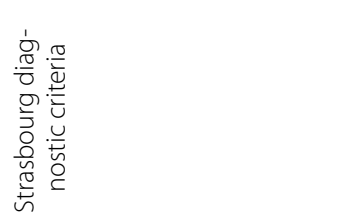 & 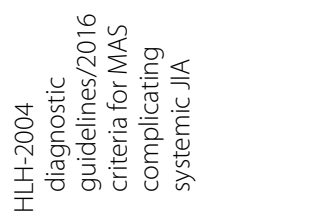 \\
\hline 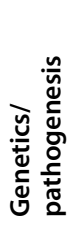 & 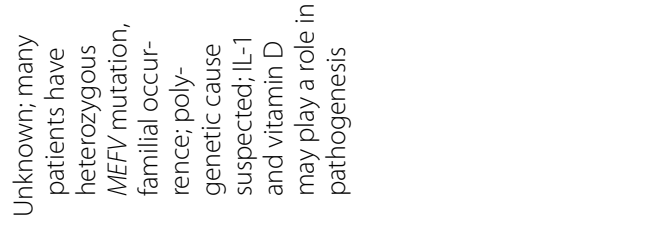 & 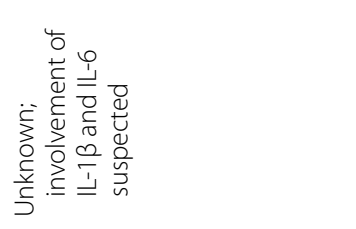 & 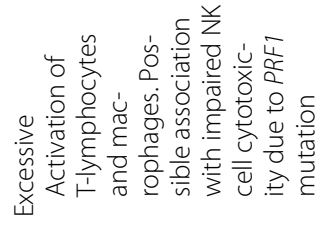 \\
\hline & 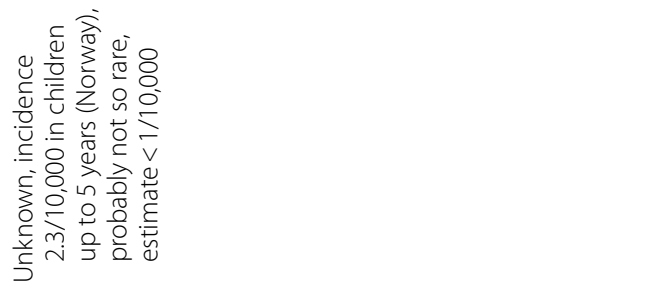 & 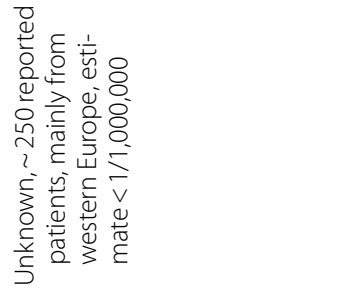 & 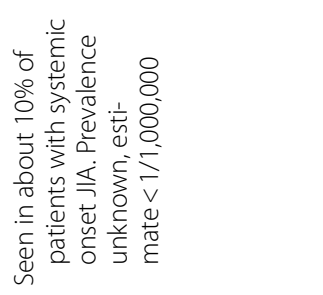 \\
\hline 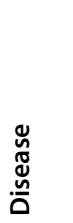 & 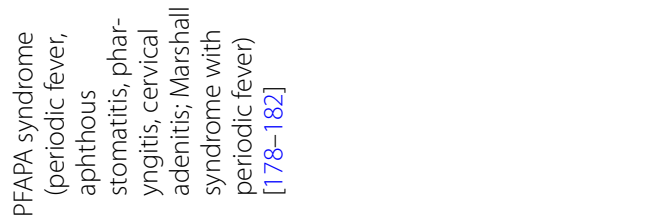 & 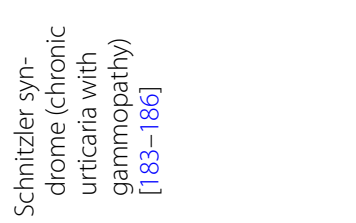 & 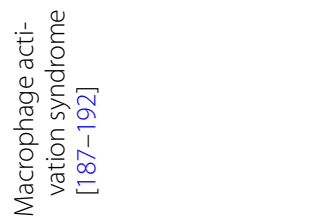 \\
\hline
\end{tabular}




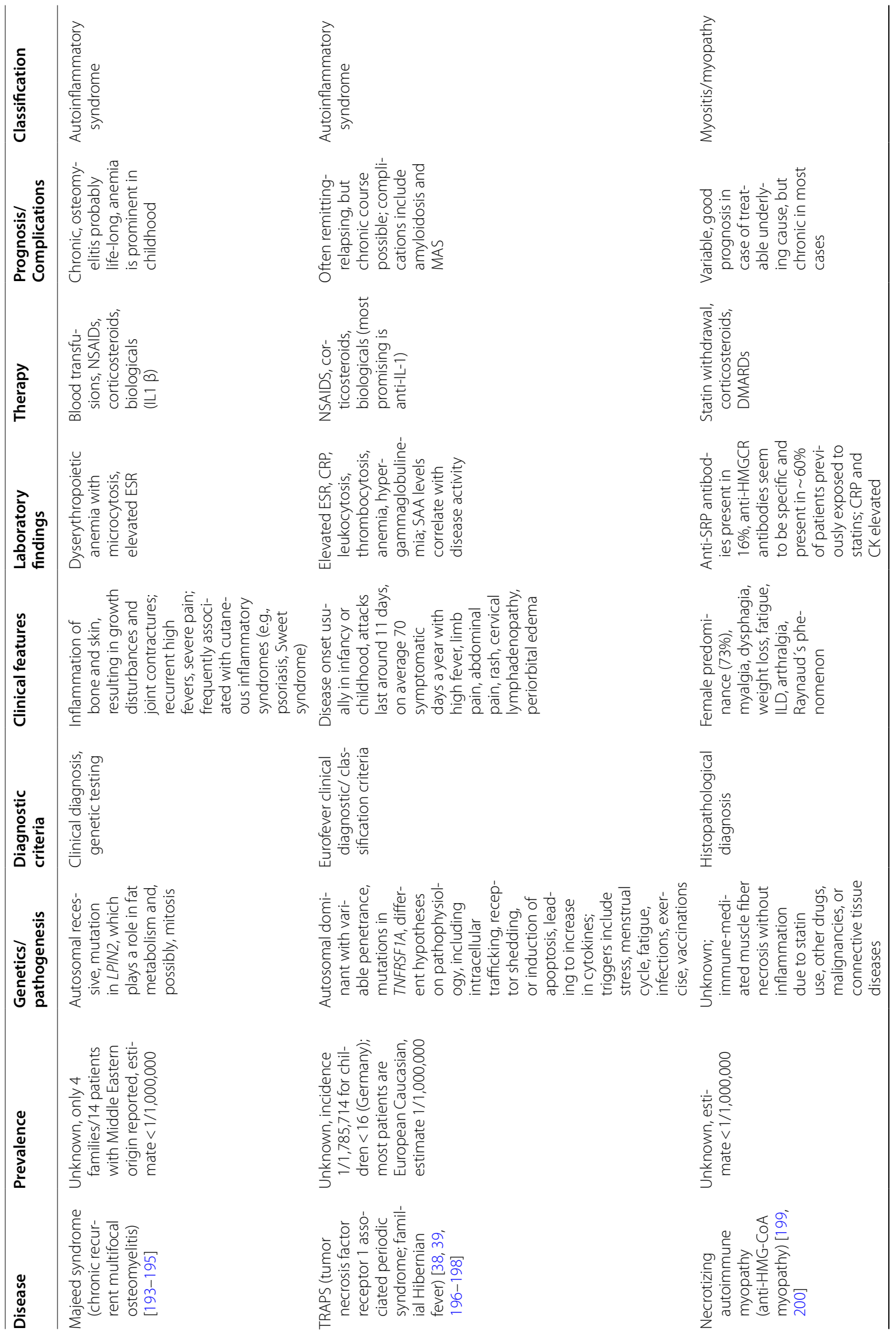




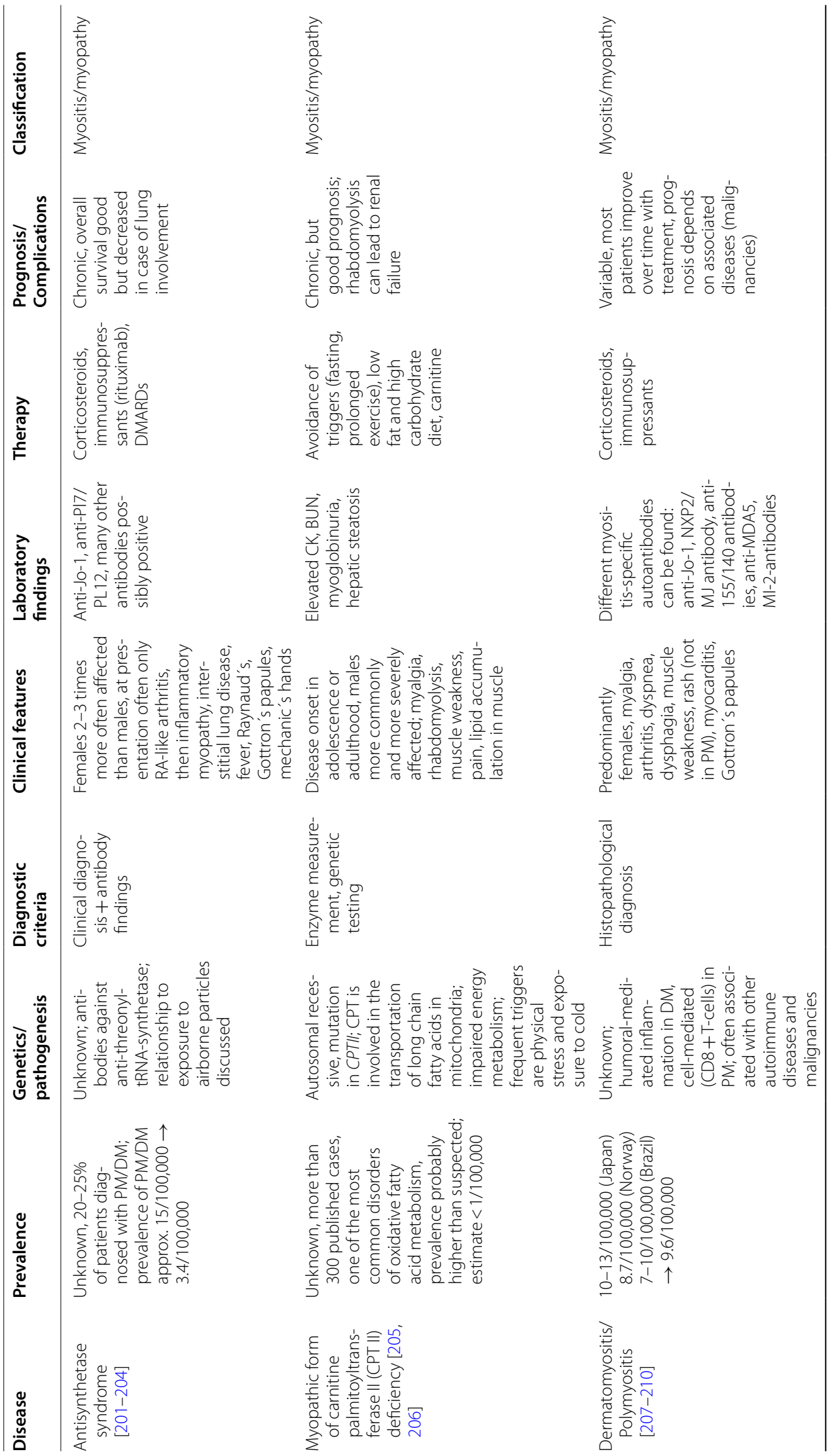




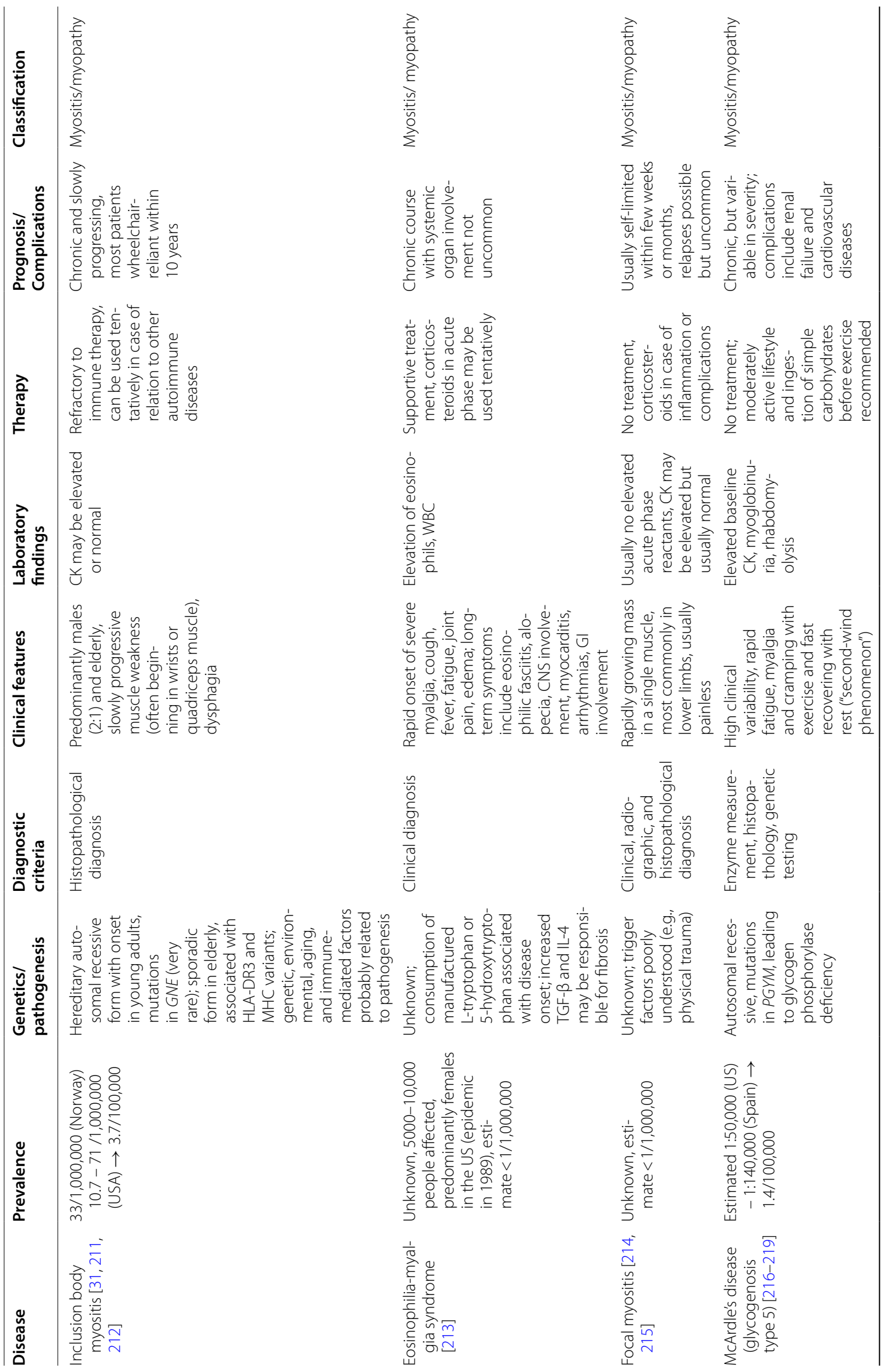




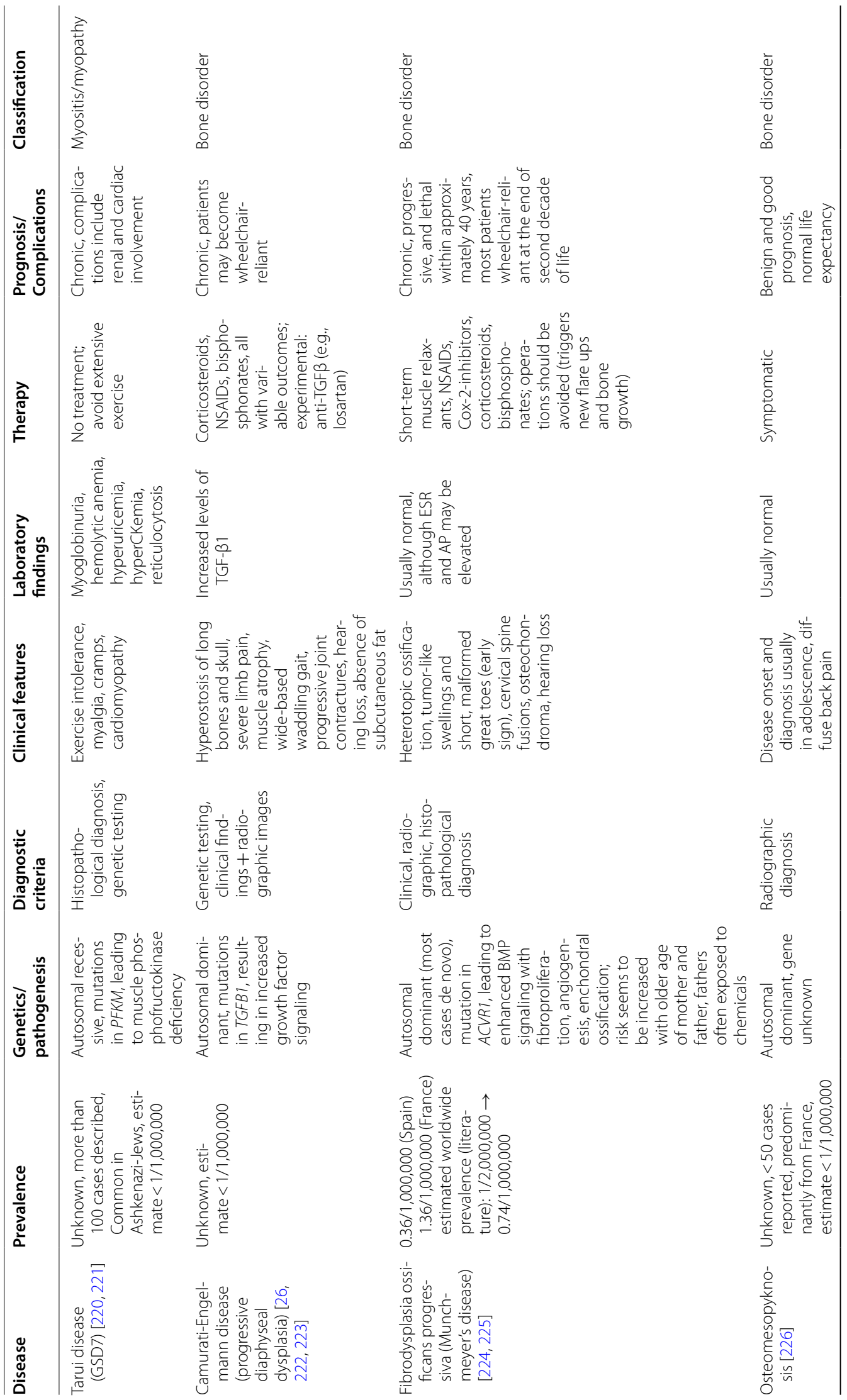




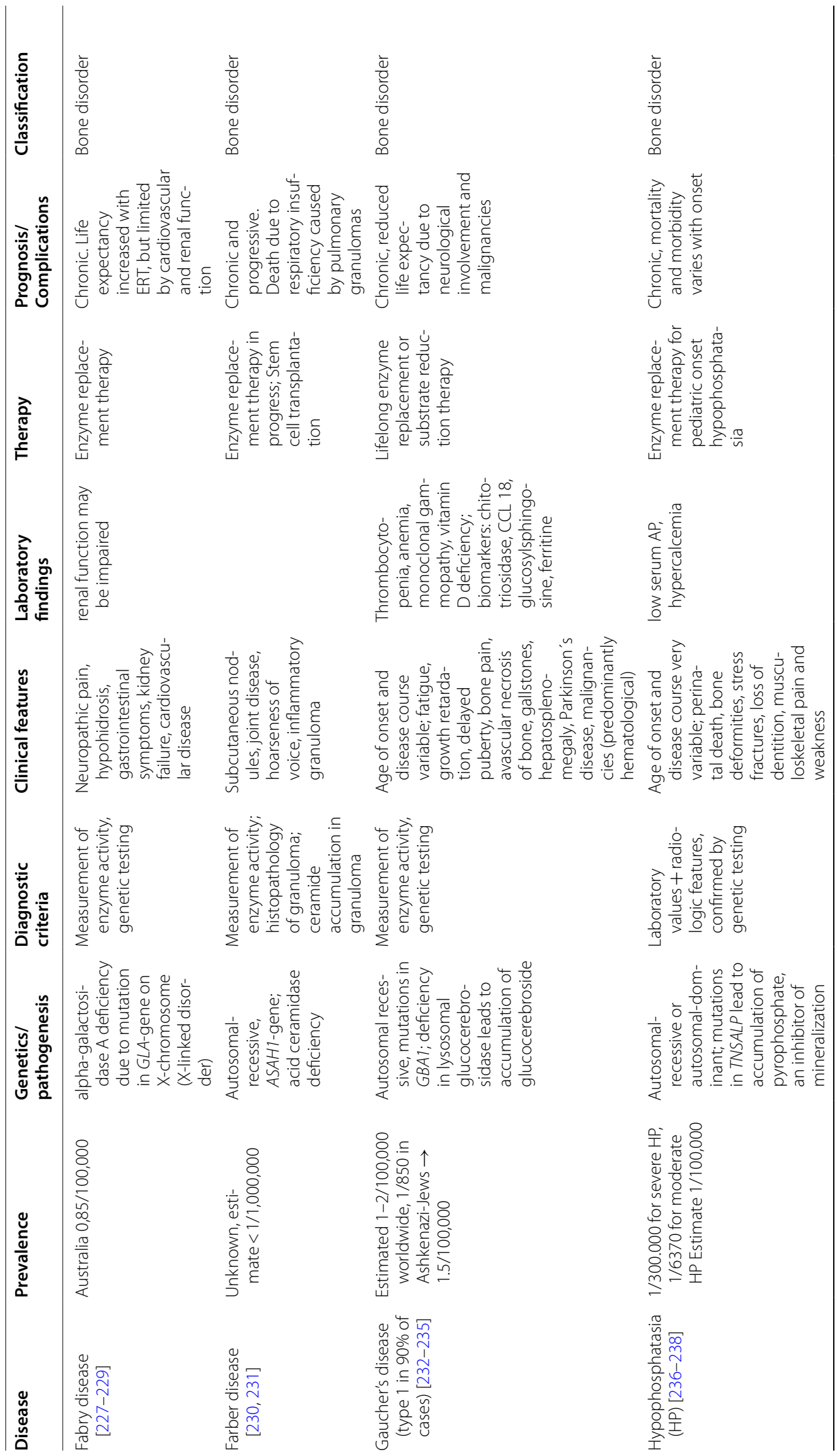




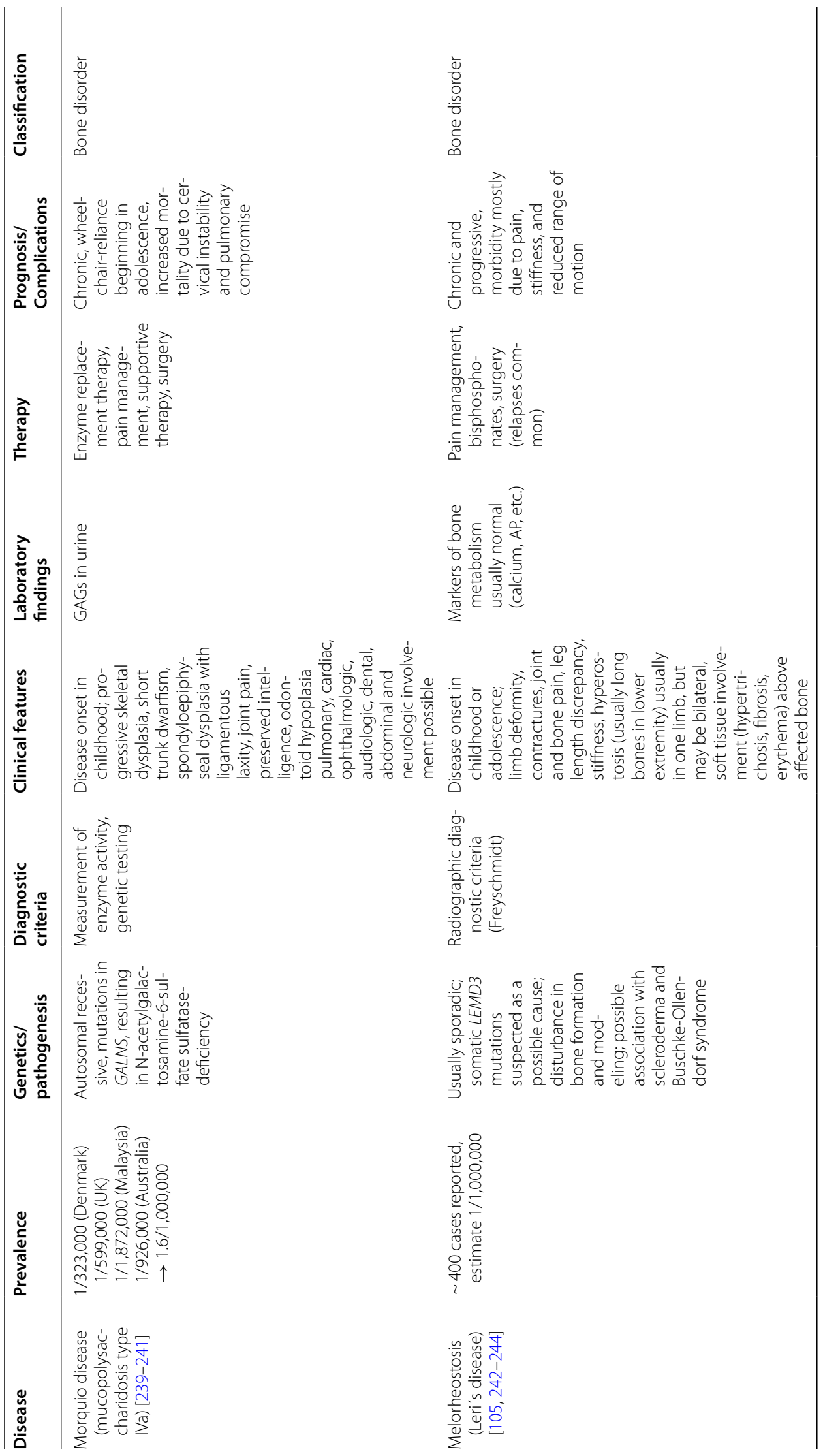




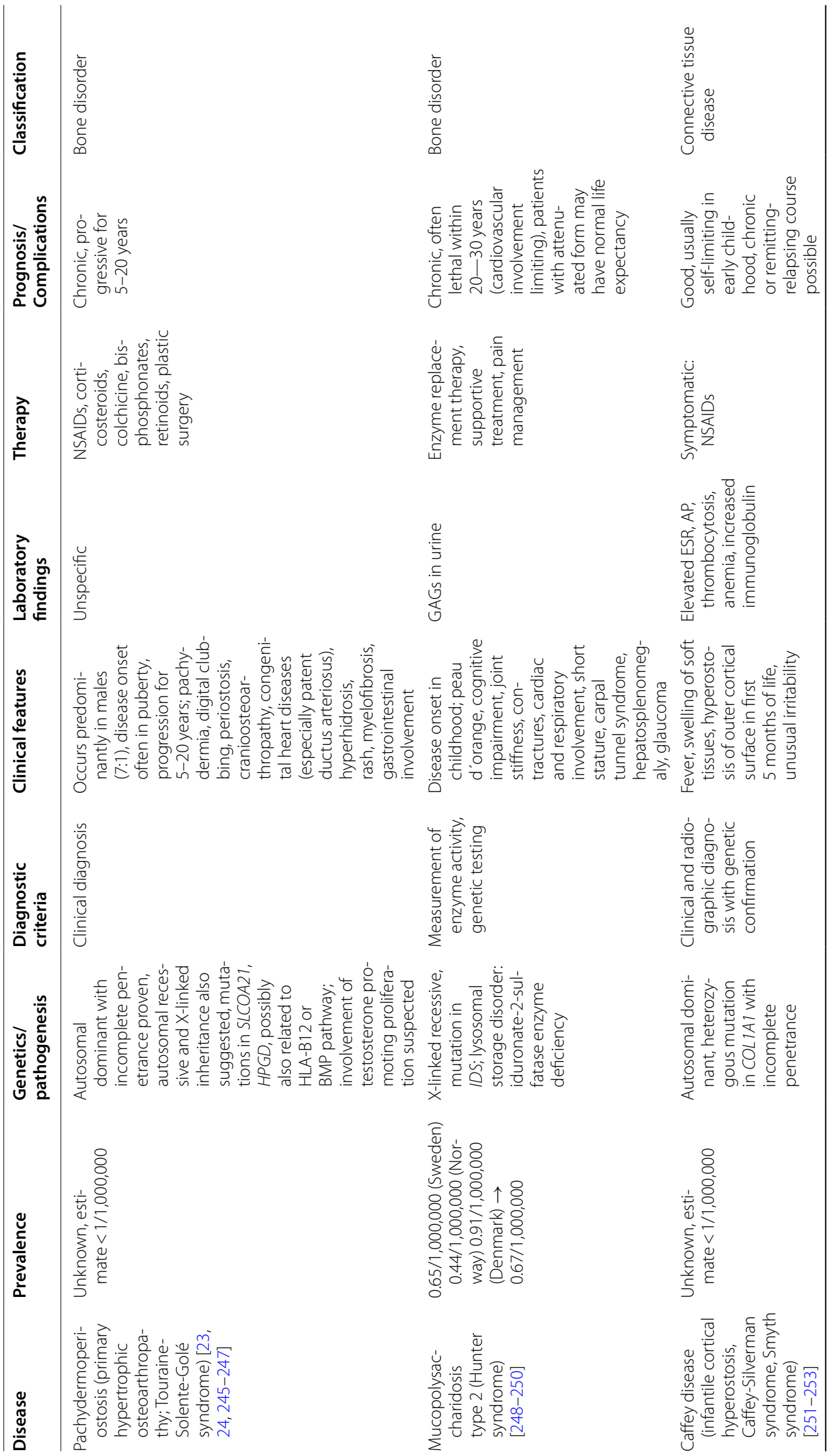




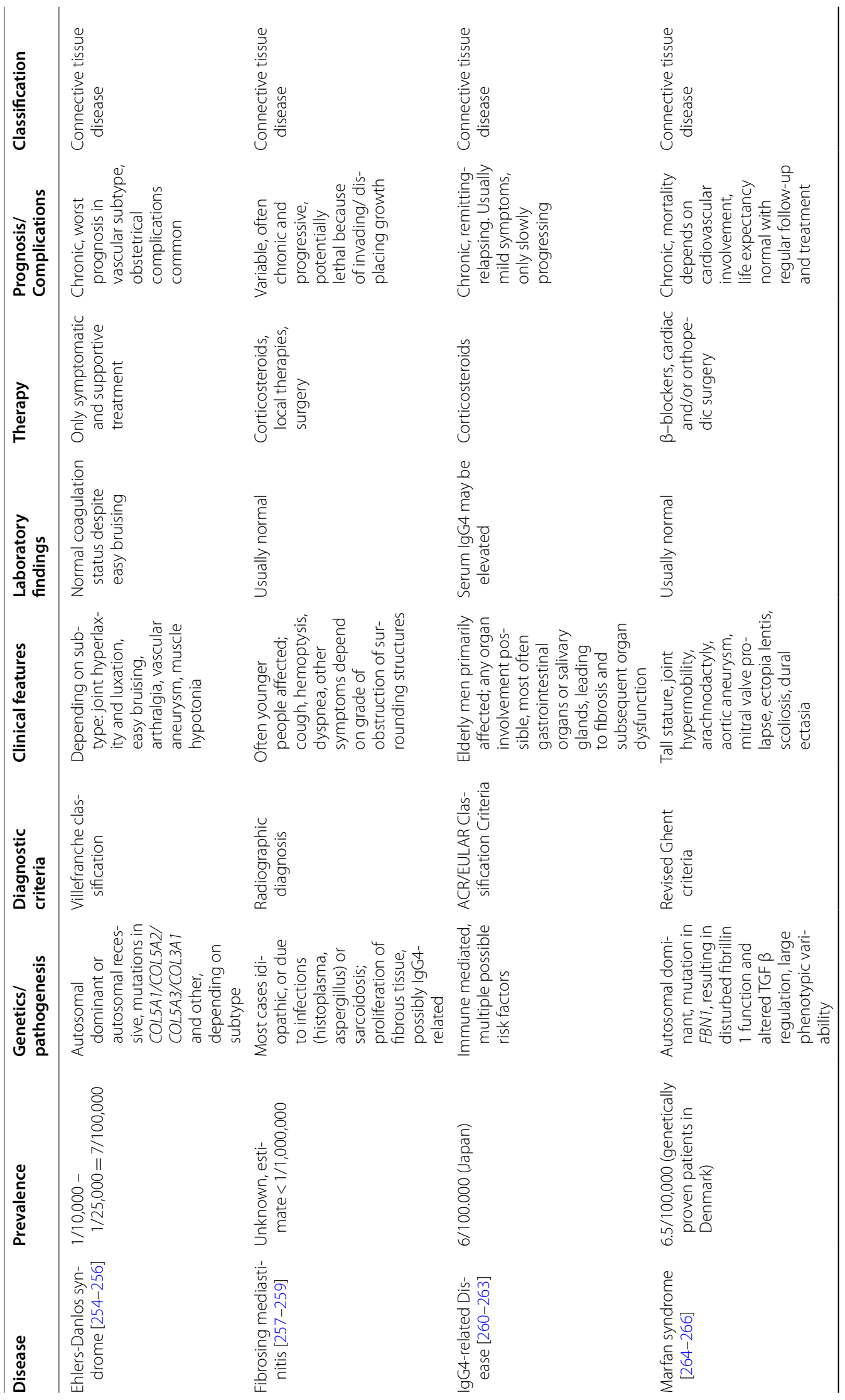




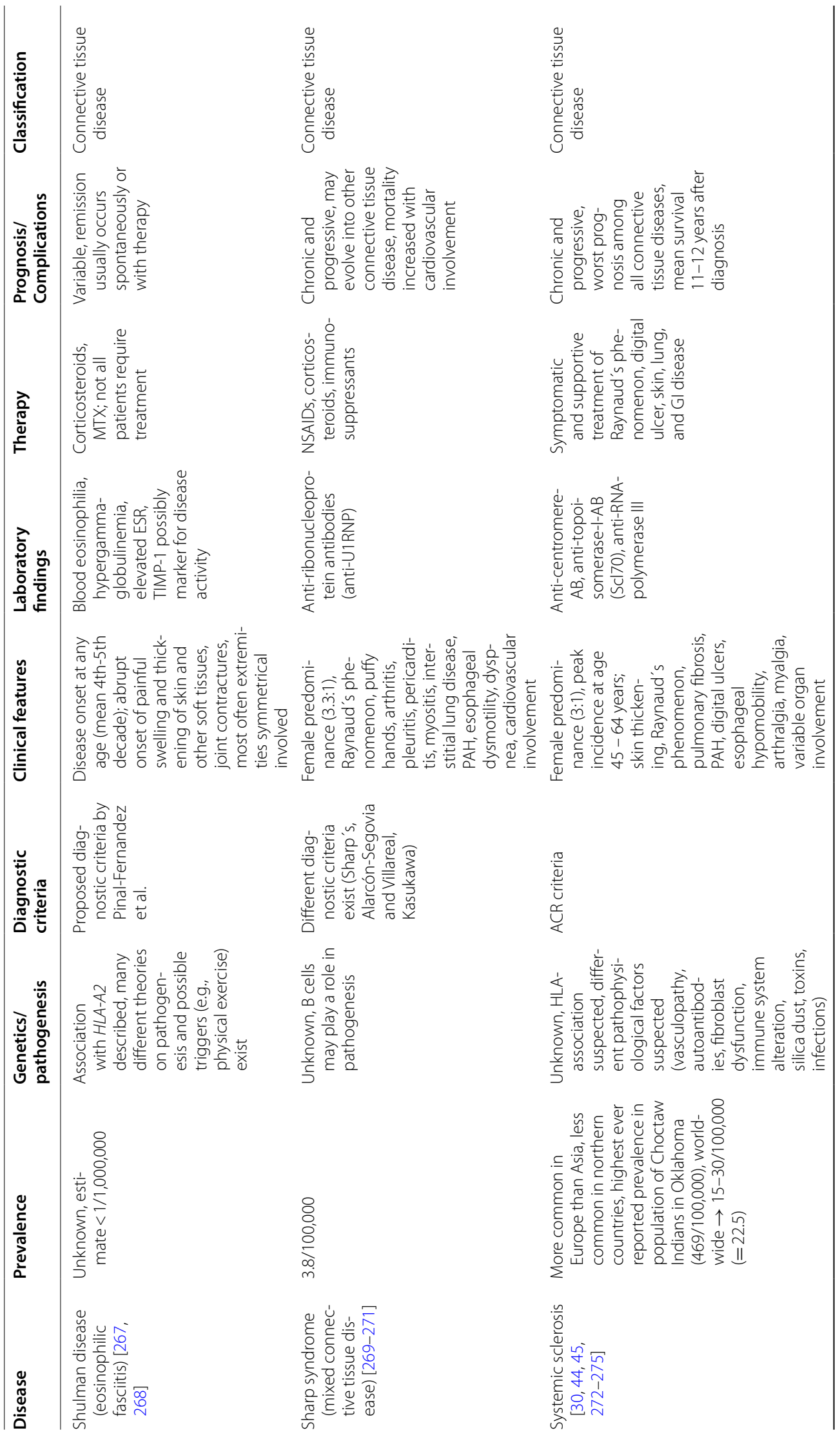




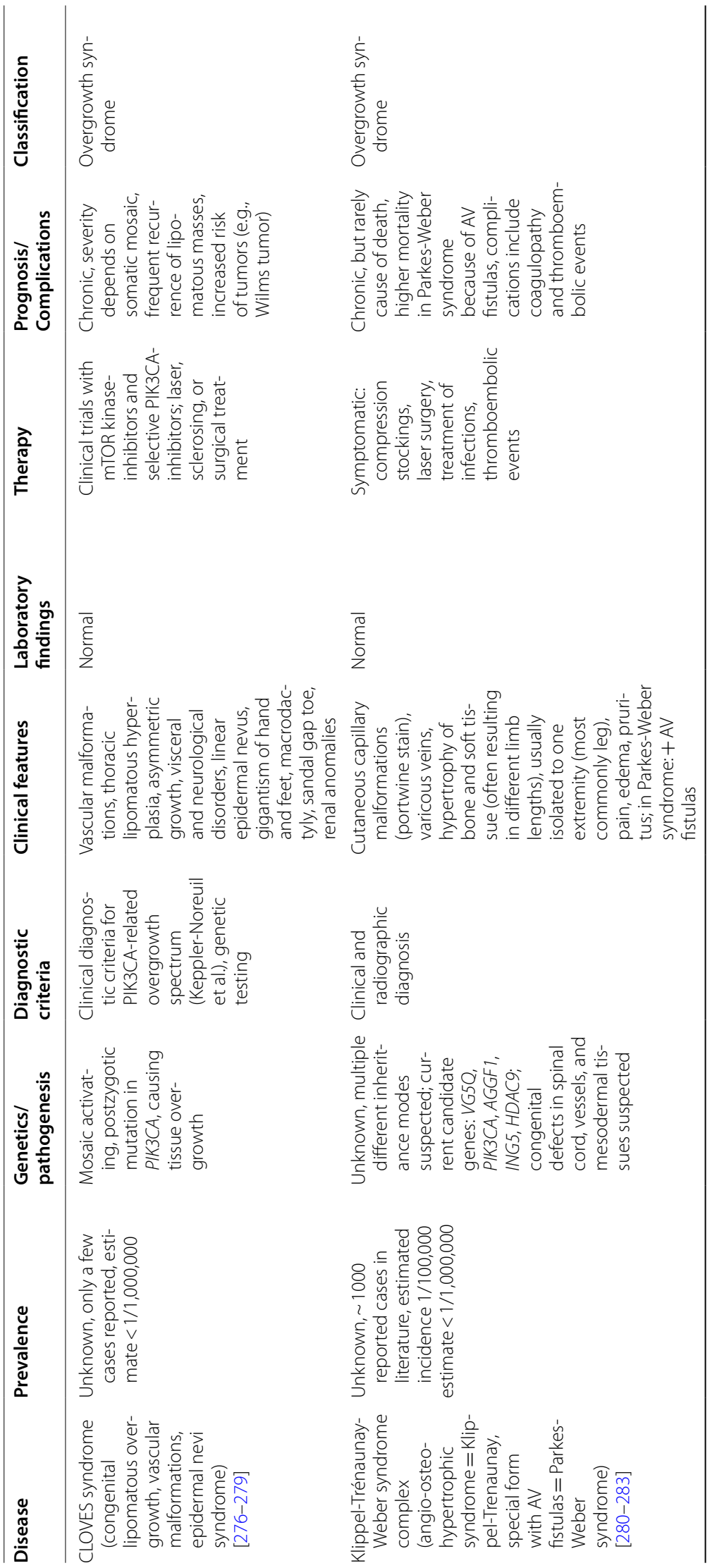




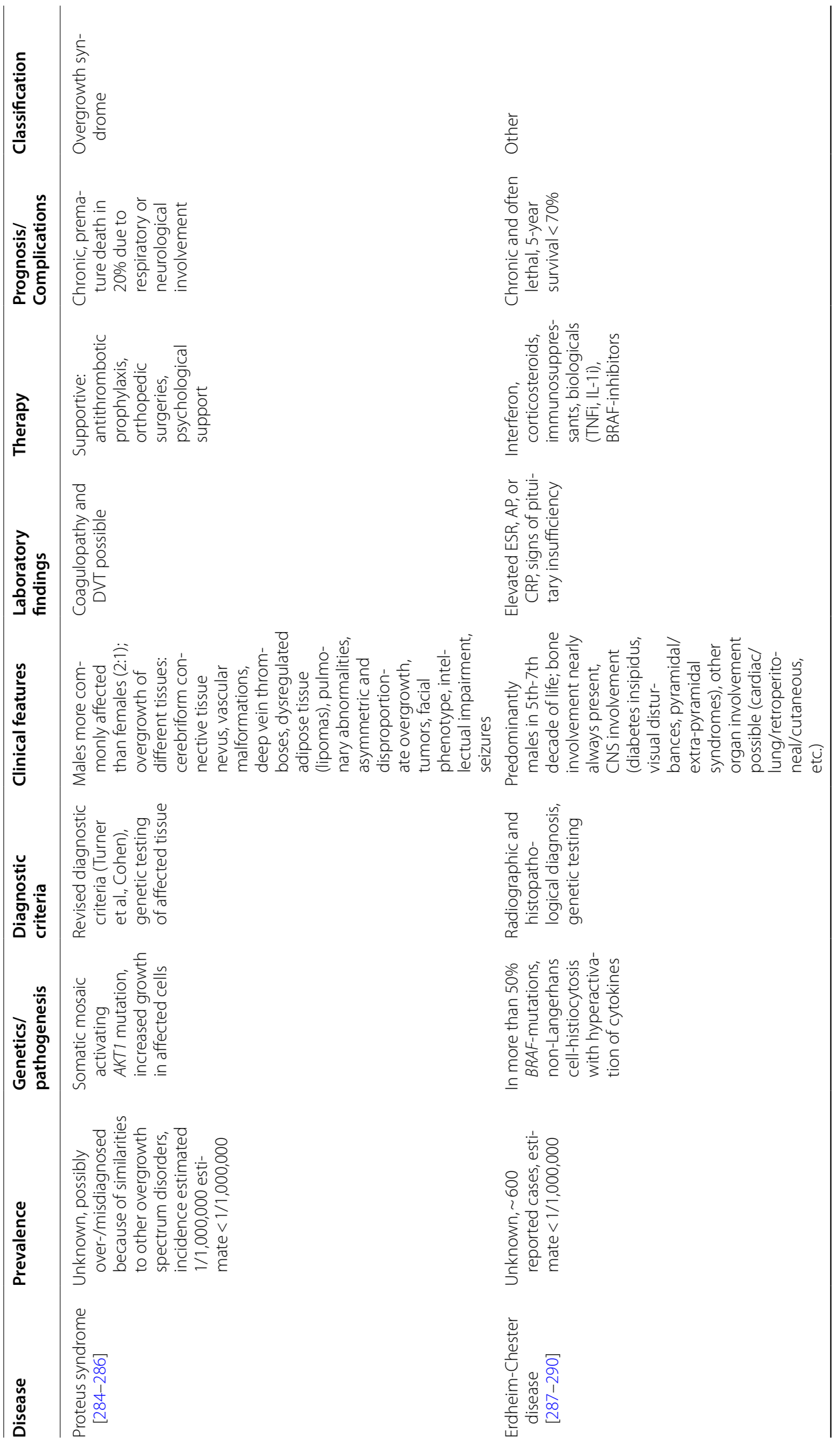




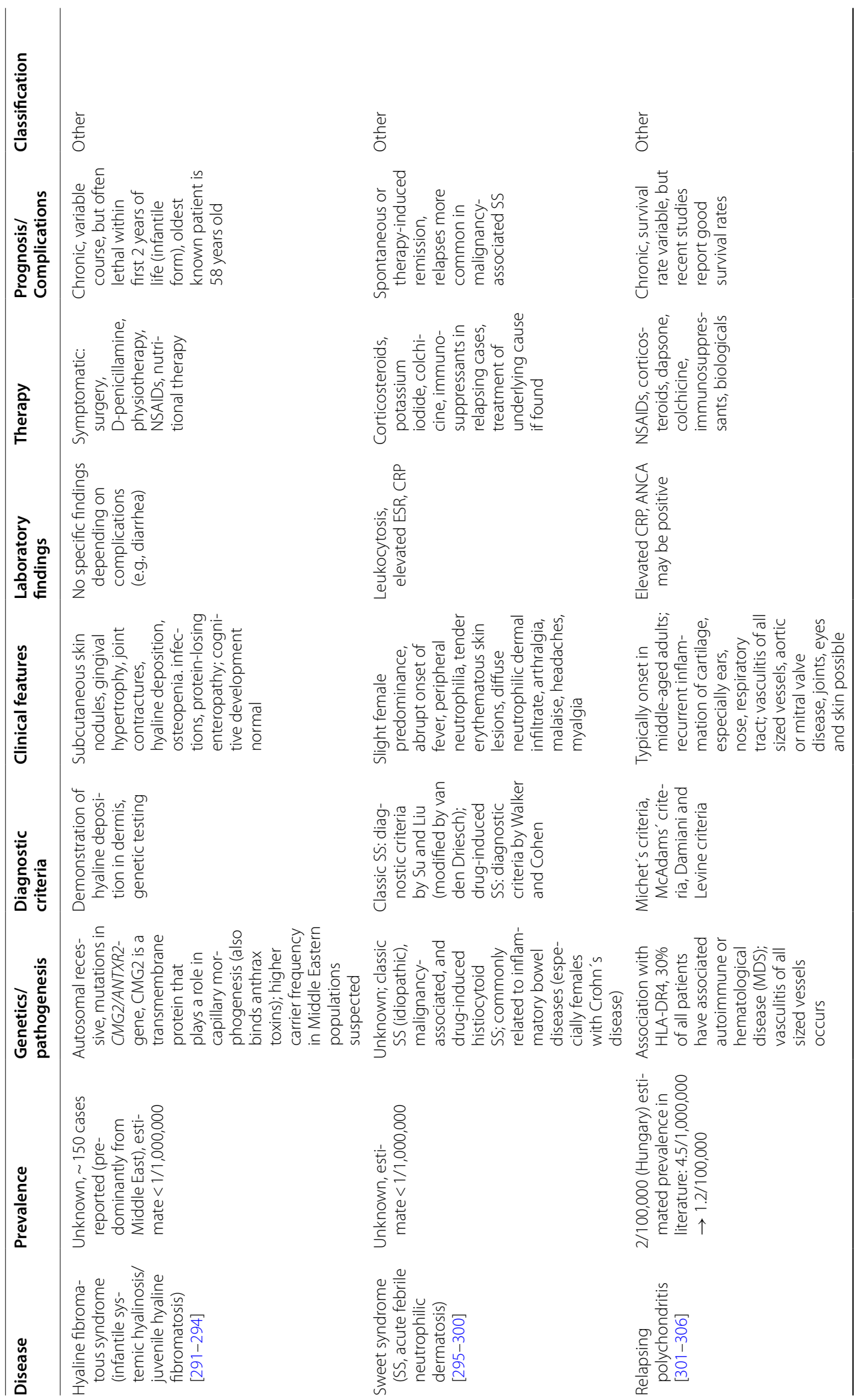




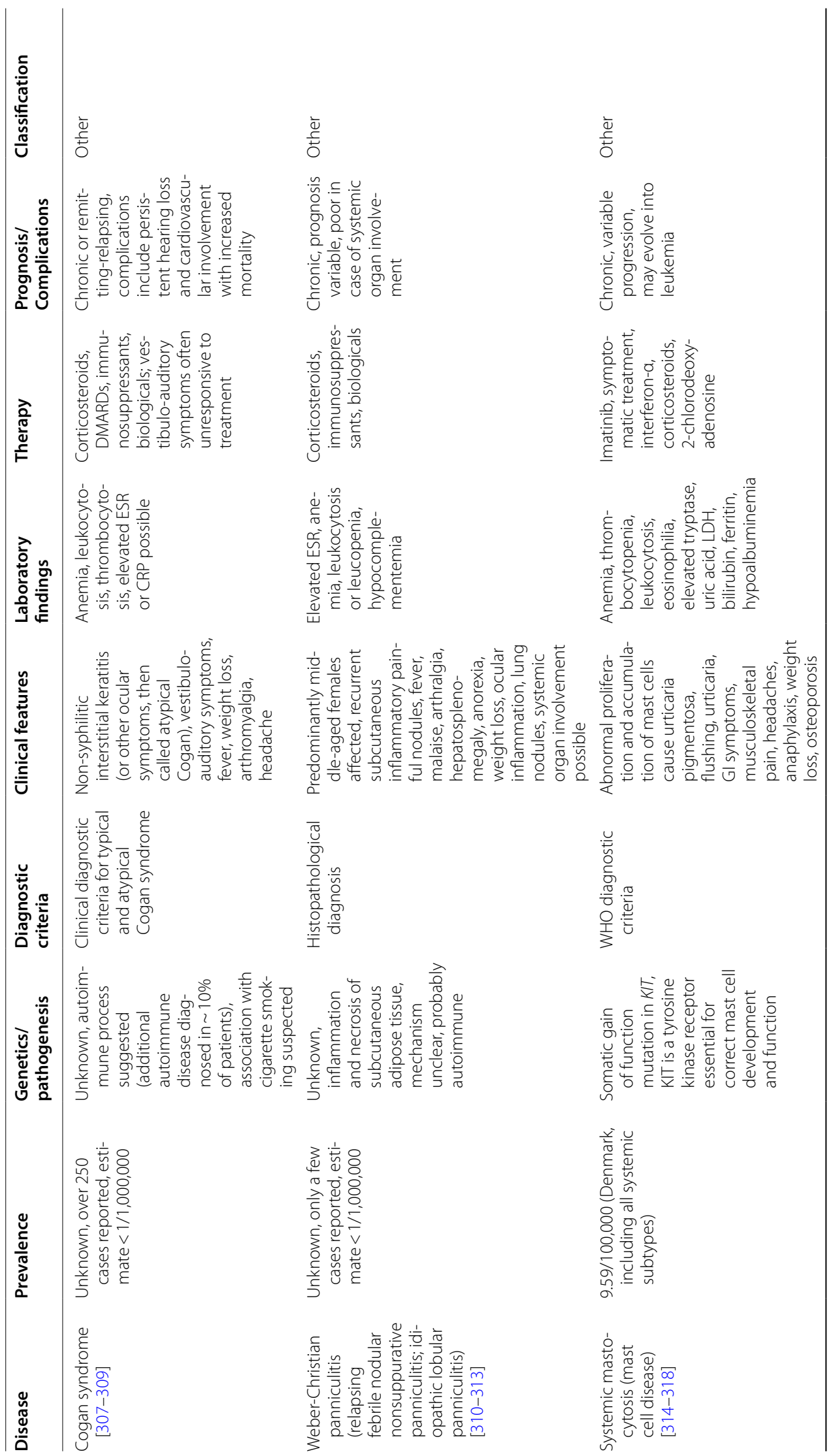




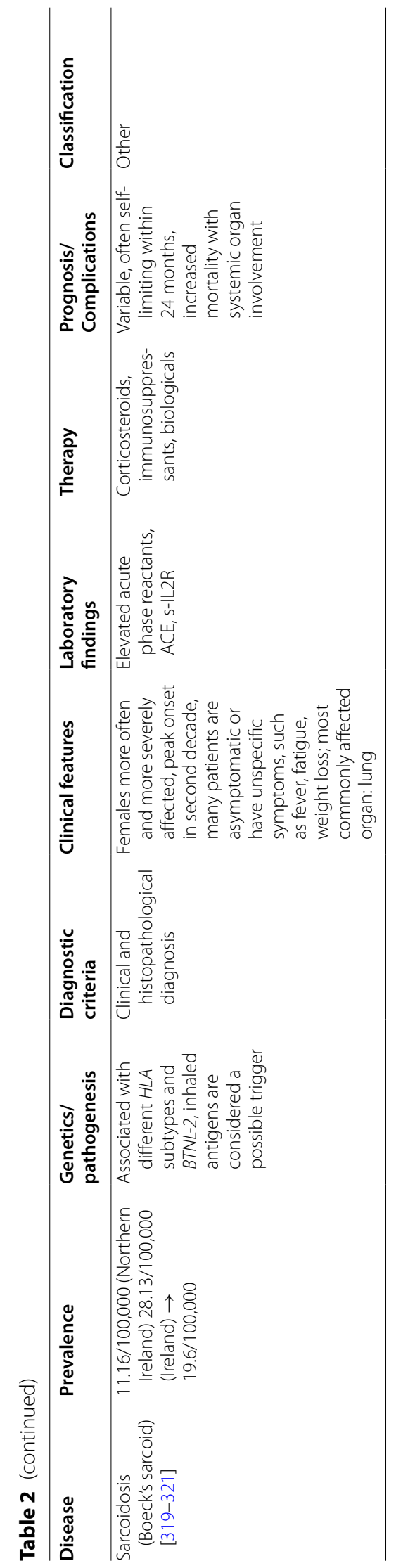




\section{Abbreviations}

AKT: Serine/threonine kinase 1; ANA: Antinuclear antibody; ANCA: Anti-neutrophil cytoplasmic antibody; ANKH: ANKH inorganic pyrophosphate transport regulator; ANTRX2: Anthrax toxin receptor 2; AP: Alkaline phosphatase; BMP: Bone morphogenetic protein; BUN: Blood urea nitrogen; CAPS: Cryoporinassociated periodic syndrome; CCL18: CC-chemokine ligand 18; CECR1: Cat eye syndrome chromosome region; CIAS1: Cold-induced autoinflammatory syndrome 1; CINCA: Chronic infantile neurological, cutaneous, and articular syndrome; CMG2: Capillary morphogenesis gene 2; COL1A1: Collagen type 1 alpha 1; CPTII: Carnitine palmitoyltransferase 2; CPPD: Calcium pyrophosphate dehydrate; DM: Dermatomyositis; DMARD: Disease-modifying anti-rheumatic drug; DNASE1L3: Deoxyribonuclease 1 like 3; DVT: Deep vein thrombosis; FBN1: Fibrillin 1; FCGR2A/3A: Fc fragment of IgG receptor 2A/3A; GAG: Glycosaminoglycan; GALNS: Galactosamine (N-acetyl)-6-sulfatase; GBA1: Glucocerebrosidase beta; GCSF: Granulocyte colony-stimulating factor; Gl: Gastrointestinal; GNE: Glucosamine (UDP-N-acetyl)-2-epimerase/N-acetylmannosamine kinase; HDAC9: Histone deacetylase 9; HLA: Human leukocyte antigene; HMGCoA: 3-Hydroxy-3-methylglutaryl-coenzyme A; HPGD: Hydroxyprostaglandin dehydrogenase; IDS: Iduronate 2-sulfatase; IL: Interleukin; ILAR: International League of Associations for Rheumatology; ILD: Interstitial lung disease; ING5: Inhibitor of growth family member 5; IOMID: Infantile-onset multisystem inflammatory disease; JIA: Juvenile idiopathic arthritis; KIT: KIT proto-oncogene receptor tyrosine kinase; MIF: Macrophage migration inhibitory factor; HLA: Human leukocyte antigene; MAS: Macrophage activation syndrome; MDS: Myelodysplastic syndrome; MEFV: Mediterranean fever; MIF: Macrophage migration inhibitory factor; MMP3: Matrix metalloproteinase-3; MPO: Myeloperoxidase; MVK: Mevalonate kinase; NF-kB: Nuclear factor kappa-light-chainenhancer of activated B-cells; NOD2: Nucleotide binding oligomerization domain containing 2; NOMID: Neonatal onset multisystem inflammatory disease; NSAID: Nonsteroidal anti-inflammatory drug; PAH: Pulmonary arterial hypertension; PFKM: Muscle phosphofructokinase; PGYM: Muscle glycogen phosphorylase; PIK3CA: Phosphatidylinositol-4,5-bisphosphate 3-kinase catalytic subunit A; PM: Polymyositis; PPD/PPRD: Progressive pseudorheumatoid dysplasia; PSMB8: Proteasome subunit beta 8; PSTPIP1: Prolineserine-threonine phosphatase interacting protein 1; PTX3: Pentraxine 3; RF: Rheumatoid factor; RA: Rheumatoid arthritis; SAA: Serum amyloid A; SAPHO: Synovitis-acne-pustulosis-hyperostosis-osteitis-syndrome; SEDT-PA: Spondyloepiphyseal dysplasia tarda with progressive arthropathy; SLE: Systemic lupus erythematosus; TGFB1: Transforming growth factor; TIMP1: Tissue inhibitor of metalloproteinase; TNFi: Tumor necrosis factor inhibitor; TNFRSF: Tumor necrosis factor receptor superfamily; VEGF: Vascular endothelial growth factor; WBC: White blood cell; WISP3: WNT1 inducible signaling pathway protein.

\section{Acknowledgements}

San Francisco edit for editing and proofreading the manuscript.

\section{Authors' contributions}

MM, CS and MS conceptualized the work and substantively revised it. JL, TB, and CD acquired and analyzed the data. JL, DK and MS analyzed and interpreted the data, and wrote the manuscript. All authors read and approved the final manuscript.

\section{Funding}

Open Access funding enabled and organized by Projekt DEAL. This research did not receive any specific grant from funding agencies in the public, commercial, or not-for-profit sectors.

\section{Availability of data and materials}

All data generated or analysed during this study are included in this published article. Data citations are included in the reference list.

\section{Declarations}

Ethics approval and consent to participate Not applicable.

\section{Consent for publication}

Not applicable.

\section{Competing interests}

The authors declare that they have no competing interests.

\section{Author details}

${ }^{1}$ Center for Rare Diseases Bonn (ZSEB), University Hospital, Bonn, Germany. ${ }^{2}$ Department of Neonatology and Pediatric Care, Children's University Hospital, Bonn, Germany. ${ }^{3}$ Institute of Human Genetics, University Hospital, Bonn, Germany. ${ }^{4}$ Institute of General Practice and Family Medicine, University Hospital, Venusberg-Campus 1, 53127 Bonn, Germany. ${ }^{5}$ Department of Radiology, University Hospital, Bonn, Germany. ${ }^{6}$ Division of Medical Psychology and Department of Psychiatry, University Hospital, Bonn, Germany. ${ }^{7}$ Department of Rheumatology, Spitalzentrum-Centre hospitalier, Biel-Bienne, Switzerland.

Received: 13 April 2021 Accepted: 2 July 2021

Published online: 22 July 2021

\section{References}

1. Windheuser IC, Mücke M, Klawonn F, Stieber C. Patients without diagnosis: A profile. (Poster Presentation 2nd World Congress on Rare Diseases and Orphan Drugs. London 2017)

2. Busch MA, Maske UE, Ryl L, Schlack R, Hapke U. Prävalenz von depressiver Symptomatik und diagnostizierter Depression bei Erwachsenen in Deutschland: Ergebnisse der Studie zur Gesundheit Erwachsener in Deutschland (DEGS1). Bundesgesundheitsblatt - Gesundheitsforschung - Gesundheitsschutz. 2013;56:733-9.

3. Okumura T, Tanno S, Ohhira M, Nozu T. The rate of polymyalgia rheumatica (PMR) and remitting seronegative symmetrical synovitis with pitting edema (RS3PE) syndrome in a clinic where primary care physicians are working in Japan. Rheumatol Int. 2012;32:1695-9.

4. Evensen KJ, Nossent HC. Epidemiology and outcome of adult-onset Still's disease in Northern Norway. Scand J Rheumatol. 2006;35:48-51.

5. Balci MA, Pamuk ON, Pamuk GE, Uzundere FK, Donmez S. AB1142 epidemiology and outcome of adult-onset still9s disease in northwestern thrace region in Turkey. Ann Rheum Dis. 2015;74:1284-1284.

6. Asanuma YF, Mimura T, Tsuboi H, Noma H, Miyoshi F, Yamamoto K, et al. Nationwide epidemiological survey of 169 patients with adult Still's disease in Japan. Mod Rheumatol. 2015;25:393-400.

7. Mistry PK, Cappellini MD, Lukina E, Özsan H, Pascual SM, Rosenbaum H, et al. Consensus conference: a reappraisal of Gaucher disease-diagnosis and disease management algorithms. Am J Hematol. 2011;86:110-5.

8. Miceli-Richard C, Lesage S, Rybojad M, Prieur A-M, Manouvrier-Hanu $\mathrm{S}$, Häfner R, et al. CARD15 mutations in Blau syndrome. Nat Genet. 2001;29:19-20.

9. Wouters CH, Maes A, Foley KP, Bertin J, Rose CD. Blau Syndrome, the prototypic auto-inflammatory granulomatous disease. Pediatr Rheumatol Online J. 2014;12:33.

10. Zhong Y, Kinio A, Saleh M. Functions of NOD-like receptors in human diseases. Front Immunol [Internet]. 2013 [cited 2018 Aug 29];4. Available from: https://www.ncbi.nlm.nih.gov/pmc/articles/PMC3797414/

11. Levy R, Gérard L, Kuemmerle-Deschner J, Lachmann HJ, Koné-Paut I, Cantarini L, et al. Phenotypic and genotypic characteristics of cryopyrin-associated periodic syndrome: a series of 136 patients from the Eurofever Registry. Ann Rheum Dis. 2015;74:2043-9.

12. Hoffman HM, Mueller JL, Broide DH, Wanderer AA, Kolodner RD. Mutation of a new gene encoding a putative pyrin-like protein causes familial cold autoinflammatory syndrome and Muckle-Wells syndrome. Nat Genet. 2001;29:301-5.

13. Consortium TFF, Bernot A, Clepet C, Dasilva C, Devaud C, Petit J-L, et al. A candidate gene for familial Mediterranean fever. Nat Genet. 1997; 17:25-31.

14. The International FMF Consortium. Ancient missense mutations in a new member of the RoRet gene family are likely to cause familial mediterranean fever. Cell. 1997;90:797-807.

15. Celiksoy MH, Ogur G, Yaman E, Abur U, Fazla S, Sancak R, et al. Could familial Mediterranean fever gene mutations be related to PFAPA syndrome? Pediatr Allergy Immunol. 2016;27:78-82.

16. Taniuchi S, Nishikomori R, Iharada A, Tuji S, Heike T, Kaneko K. MEFV variants in patients with PFAPA syndrome in Japan. Open Rheumatol J. 2013;7:22-5. 
17. Nonaka F, Migita K, Jiuchi Y, Shimizu T, Umeda M, Iwamoto N, et al. Increased prevalence of MEFV exon 10 variants in Japanese patients with adult-onset Still's disease. Clin Exp Immunol. 2015;179:392-7.

18. Yim D, Curtis N, Cheung M, Burgner D. Update on Kawasaki disease: epidemiology, aetiology and pathogenesis. J Paediatr Child Health. 2013;49:704-8.

19. Rodó X, Ballester J, Cayan D, Melish ME, Nakamura Y, Uehara R, et al. Association of Kawasaki disease with tropospheric wind patterns. Sci Rep [Internet]. 2011 [cited 2018 Aug 30];1. Available from: https:// www.ncbi.nlm.nih.gov/pmc/articles/PMC3240972/

20. Yan M, Wang Z, Niu N, Zhao J, Peng J. Relationship between chronic tonsillitis and Henoch-Schonlein purpura. Int J Clin Exp Med. 2015:8:14060-4.

21. Piram M, Mahr A. Epidemiology of immunoglobulin A vasculitis (Henoch-Schönlein): current state of knowledge. Curr Opin Rheumatol. 2013;25:171-8.

22. Bianchi L, Lubrano C, Carrozzo AM, Iraci S, Tomassoli M, Spera G, et al. Pachydermoperiostosis: study of epidermal growth factor and steroid receptors. Br J Dermatol. 1995;132:128-33.

23. Zhang Z, Xia W, He J, Zhang Z, Ke Y, Yue H, et al. Exome sequencing identifies SLCO2A1 mutations as a cause of primary hypertrophic osteoarthropathy. Am J Hum Genet. 2012;90:125-32.

24. Castori M, Sinibaldi L, Mingarelli R, Lachman R, Rimoin D, Dallapiccola B. Pachydermoperiostosis: an update. Clin Genet. 2005:68:477-86.

25. Xie G, Jiang N, Liang C, Zeng J, Chen Z, Xu Q, et al. Pigmented Villonodular Synovitis: A Retrospective Multicenter Study of 237 Cases. PLoS One [Internet]. 2015 [cited 2017 Sep 8];10. Available from: http://www.ncbi.nlm.nih.gov/pmc/articles/PMC4370558/

26. Janssens K, Vanhoenacker F, Bonduelle M, Verbruggen L, Van Maldergem L, Ralston S, et al. Camurati-Engelmann disease: review of the clinical, radiological, and molecular data of 24 families and implications for diagnosis and treatment. J Med Genet. 2006;43:1-11.

27. Kadavath S, Efthimiou P. Adult-onset Still's disease-pathogenesis, clinical manifestations, and new treatment options. Ann Med. 2015:47:6-14.

28. Smith EJ, Allantaz F, Bennett L, Zhang D, Gao X, Wood G, et al. Clinical, molecular, and genetic characteristics of PAPA syndrome: a review. Curr Genom. 2010;11:519-27.

29. Comarmond C, Cacoub P. Granulomatosis with polyangiitis (Wegener): clinical aspects and treatment. Autoimmun Rev. 2014;13:1121-5.

30. Kowal-Bielecka O, Fransen J, Avouac J, Becker M, Kulak A, Allanore Y, et al. Update of EULAR recommendations for the treatment of systemic sclerosis. Ann Rheum Dis. 2017;76:1327-39.

31. Needham M, Mastaglia FL. Inclusion body myositis: current pathogenetic concepts and diagnostic and therapeutic approaches. Lancet Neurol. 2007;6:620-31.

32. Karmacharya P, Donato AA, Aryal MR, Ghimire S, Pathak R, Shah K, et al. RS3PE revisited: a systematic review and meta-analysis of 331 cases. Clin Exp Rheumatol. 2016:34:404-15.

33. Hertzman PA. The eosinophilia-myalgia syndrome: status of 205 patients and results of treatment 2 years after onset. Ann Intern Med. 1995;122:851.

34. Stone JH, Merkel PA, Spiera R, Seo P, Langford CA, Hoffman GS, et al. Rituximab versus cyclophosphamide for ANCA-associated vasculitis. N E J Med. 2010;363:221-32.

35. Renko M, Salo E, Putto-Laurila A, Saxen H, Mattila PS, Luotonen J, et al. A randomized, controlled trial of tonsillectomy in periodic fever, aphthous stomatitis, pharyngitis, and adenitis syndrome. J Pediatr. 2007;151:289-92.

36. Ben-Zvi I, Kukuy O, Giat E, Pras E, Feld O, Kivity S, et al. Anakinra for colchicine-resistant familial mediterranean fever: a randomized, double-blind, placebo-controlled trial. Arthritis Rheumatol. 2017;69:854-62.

37. Tariq S, Hugenberg ST, Hirano-Ali SA, Tariq H. Multicentric reticulohistiocytosis $(\mathrm{MRH})$ : case report with review of literature between 1991 and 2014 with in depth analysis of various treatment regimens and outcomes. Springerplus [Internet]. 2016 [cited 2017 Sep 13];5. Available from: http://www.ncbi.n/m.nih.gov/pmc/articles/PMC4766148/

38. Federici S, Sormani MP, Ozen S, Lachmann HJ, Amaryan G, Woo P, et al. Evidence-based provisional clinical classification criteria for autoinflammatory periodic fevers. Ann Rheum Dis. 2015;74:799-805.
39. ter Haar N, Lachmann H, Özen S, Woo P, Uziel Y, Modesto C, et al. Treatment of autoinflammatory diseases: results from the Eurofever Registry and a literature review. Ann Rheum Dis. 2013;72:678-85.

40. Rai E, Mahajan A, Kumar P, Angural A, Dhar MK, Razdan S, et al. Whole Exome Screening Identifies Novel and Recurrent WISP3 Mutations Causing Progressive Pseudorheumatoid Dysplasia in Jammu and Kashmir-India. Sci Rep [Internet]. 2016 [cited 2017 Jun 23];6. Available from: http://www.ncbi.nlm.nih.gov/pmc/articles/PMC4904372/

41. Adeeb F, Stack AG, Fraser AD. Knitting the Threads of Silk through Time: Behçet's Disease_-Past, Present, and Future. Int J Rheumatol [Internet]. 2017 [cited 2017 Nov 30];2017. Available from: https://www.ncbi.nlm. nih.gov/pmc/articles/PMC5610876/

42. Ben-Chetrit E, Touitou I. Familial mediterranean fever in the world Arthritis Rheum. 2009:61:1447-53.

43. Monti G, Saccardo F, Castelnovo L, Novati P, Sollima S, Riva A, et al. Prevalence of mixed cryoglobulinaemia syndrome and circulating cryoglobulins in a population-based survey: the Origgio study. Autoimmun Rev. 2014;13:609-14.

44. Arnett FC, Howard RF, Tan F, Moulds JM, Bias WB, Durban E, et al. Increased prevalence of systemic sclerosis in a native american tribe in Oklahoma. Association with an Amerindian HLA haplotype. Arthritis Rheum. 1996;39:1362-70.

45. Barnes J, Mayes MD. Epidemiology of systemic sclerosis: incidence, prevalence, survival, risk factors, malignancy, and environmental triggers. Curr Opin Rheumatol. 2012;24:165-70.

46. Yates M, Graham K, Watts RA, MacGregor AJ. The prevalence of giant cell arteritis and polymyalgia rheumatica in a UK primary care population. BMC Musculoskelet Disord [Internet]. 2016 [cited 2017 Dec 4];17. Available from: https://www.ncbi.nlm.nih.gov/pmc/articles/PMC49 46178/

47. Stojan G, Petri M. Epidemiology of systemic lupus erythematosus: an update. Curr Opin Rheumatol. 2018;30:144-50.

48. Tani C, D'Aniello D, Sedie AD, Carli L, Cagnoni M, Possemato N, et al. Rhupus syndrome: Assessment of its prevalence and its clinical and instrumental characteristics in a prospective cohort of 103 SLE patients. Autoimmun Rev. 2013;12:537-41.

49. Vallet H, Riviere S, Sanna A, Deroux A, Moulis G, Addimanda O, et al. Efficacy of anti-TNF alpha in severe and/or refractory Behçet's disease: multicenter study of 124 patients. J Autoimmun. 2015;62:67-74.

50. Descriptive Epidemiology of Kawasaki Disease in Japan. 2011-2012: from the results of the 22nd Nationwide Survey. J Epidemiol. 2015;25:239-45.

51. Exarchou S, Lindström U, Askling J, Eriksson JK, Forsblad-d'Elia H, Neovius $M$, et al. The prevalence of clinically diagnosed ankylosing spondylitis and its clinical manifestations: a nationwide register study. Arthritis Res Ther [Internet]. 2015 [cited 2018 Sep 25];17. Available from: https://www.ncbi.nlm.nih.gov/pmc/articles/PMC4424886/

52. Arkkila PE. Thromboangiitis obliterans (Buerger's disease). Orphanet J Rare Dis. 2006:1:14.

53. Zheng J-F, Chen Y-M, Chen D-Y, Lin C-H, Chen H-H. The incidence and prevalence of thromboangiitis obliterans in Taiwan: a nationwide, population-based analysis of data collected from 2002 to 2011. Clinics (Sao Paulo). 2016;71:399-403.

54. Puéchal $X$, Fiessinger J-N. Thromboangiitis obliterans or Buerger's disease: challenges for the rheumatologist. Rheumatology (Oxford). 2007;46:192-9.

55. Shionoya S. Diagnostic criteria of Buerger's disease. Int J Cardiol. 1998:66:S243-5.

56. Olin JW. Thromboangiitis Obliterans (Buerger's Disease). N E J Med. 2000;343:864-9.

57. Sada K-E, Amano K, Uehara R, Yamamura M, Arimura Y, Nakamura Y, et al. A nationwide survey on the epidemiology and clinical features of eosinophilic granulomatosis with polyangiitis (Churg-Strauss) in Japan. Mod Rheumatol. 2014;24:640-4.

58. Mahr A, Guillevin L, Poissonnet M, Aymé S. Prevalences of polyarteritis nodosa, microscopic polyangiitis, Wegener's granulomatosis, and Churg-Strauss syndrome in a French urban multiethnic population in 2000: A capture-recapture estimate. Arthritis Rheum. 2004;51:92-9.

59. Mohammad AJ, Jacobsson LTH, Mahr AD, Sturfelt G, Segelmark M. Prevalence of Wegener's granulomatosis, microscopic polyangiitis, 
polyarteritis nodosa and Churg-Strauss syndrome within a defined population in southern Sweden. Rheumatology (Oxford). 2007:46:1329-37.

60. Comarmond C, Pagnoux C, Khellaf M, Cordier J-F, Hamidou M, Viallard $J-F$, et al. Eosinophilic granulomatosis with polyangiitis (Churg-Strauss): Clinical characteristics and long-term followup of the 383 patients enrolled in the French Vasculitis Study Group cohort. Arthritis Rheum. 2013;65:270-81.

61. Mahr A, Moosig F, Neumann T, Szczeklik W, Taillé C, Vaglio A, et al. Eosinophilic granulomatosis with polyangiitis (churg-strauss): evolutions in classification, etiopathogenesis, assessment and management. Curr Opin Rheumatol. 2014;26:16-23.

62. Greco A, Rizzo MI, De Virgilio A, Gallo A, Fusconi M, Ruoppolo G, et al. Churg-Strauss syndrome. Autoimmun Rev. 2015;14:341-8.

63. Masi AT, Hunder GG, Lie JT, Michel BA, Bloch DA, Arend WP, et al. The American College of Rheumatology 1990 criteria for the classification of churg-strauss syndrome (allergic granulomatosis and angiitis). Arthritis Rheum. 1990;33:1094-100.

64. Theodoridis A, Konstantinidou A, Makrantonaki E, Zouboulis C. Malignant and benign forms of atrophic papulosis (Köhlmeier-Degos disease): systemic involvement determines the prognosis. Br J Dermatol. 2014;170:110-5.

65. Theodoridis A, Makrantonaki E, Zouboulis CC. Malignant atrophic papulosis (Köhlmeier-Degos disease) —a review. Orphanet J Rare Dis. 2013;8:10.

66. Magro CM, Wang X, Garrett-Bakelman F, Laurence J, Shapiro LS, DeSancho MT. The effects of Eculizumab on the pathology of malignant atrophic papulosis. Orphanet J Rare Dis. 2013;8:185.

67. Shapiro LS, Toledo-Garcia AE, Farrell JF. Effective treatment of malignant atrophic papulosis (Köhlmeier-Degos disease) with treprostinil—early experience. Orphanet J Rare Dis. 2013;8:52.

68. Khalid U, Saleem T. Hughes-Stovin Syndrome. Orphanet J Rare Dis. 2011;6:15.

69. Erkan D, Yazici Y, Sanders A, Trost D, Yazici H. Is Hughes-Stovin syndrome Behçet's disease? Clin Exp Rheumatol. 2004;22:564-68.

70. Jachiet M, Flageul B, Deroux A, Le Quellec A, Maurier F, Cordoliani F, et al. The clinical spectrum and therapeutic management of hypocomplementemic urticarial vasculitis: data from a French Nationwide study of fifty-seven patients. Arthritis Rheumatol. 2015;67:527-34.

71. Özçakar ZB, Foster J, Diaz-Horta O, Kasapcopur O, Fan Y-S, Yalçınkaya F, et al. DNASE1L3 mutations in hypocomplementemic urticarial vasculitis syndrome. Arthritis Rheum. 2013;65:2183-9.

72. Wakamatsu R, Watanabe H, Suzuki K, Suga N, Kitagawa W, Miura N, et al. Hypocomplementemic urticarial vasculitis syndrome is associated with high levels of serum IgG4: a clinical manifestation that mimics lgG4related disease. Intern Med. 2011:50:1109-12.

73. Schwartz HR, McDuffie FC, Black LF, Schroeter AL, Conn DL. Hypocomplementemic urticarial vasculitis: association with chronic obstructive pulmonary disease. Mayo Clin Proc. 1982;57:231-8.

74. Buck A, Christensen J, McCarty M. Hypocomplementemic urticarial vasculitis syndrome. J Clin Aesthet Dermatol. 2012;5:36-46.

75. Uehara R, Belay ED. Epidemiology of Kawasaki Disease in Asia, Europe, and the United States. J Epidemiol. 2012;22:79-85.

76. Singh S, Vignesh P, Burgner D. The epidemiology of Kawasaki disease: a global update. Arch Dis Child. 2015;100:1084-8.

77. Takahashi K, Oharaseki T, Yokouchi Y. Update on etio and immunopathogenesis of Kawasaki disease. Curr Opin Rheumatol. 2014;26:31-6

78. Arora A, Wetter DA, Gonzalez-Santiago TM, Davis MDP, Lohse CM. Incidence of leukocytoclastic vasculitis, 1996-2010: a population-based study in Olmsted County, Minnesota. Mayo Clin Proc. 2014;89:1515-24.

79. Bouiller K, Audia S, Devilliers H, Collet E, Aubriot MH, Leguy-Seguin V, et al. Etiologies and prognostic factors of leukocytoclastic vasculitis with skin involvement. Medicine (Baltimore) [Internet]. 2016 [cited 2017 Nov 30];95. Available from: https://www.ncbi.n/m.nih.gov/pmc/articles/ PMC4956825

80. Johnson E, Wetter D, Lehman J, Hand J, Davis D, Tollefson M. Leukocytoclastic vasculitis in children: clinical characteristics, subtypes, causes and direct immunofluorescence findings of 56 biopsy-confirmed cases. J Eur Acad Dermatol Venereol. 2017;31:544-9.
81. Kallenberg CGM. The diagnosis and classification of microscopic polyangiitis. J Autoimmun. 2014;48-49:90-3.

82. Greco A, De Virgilio A, Rizzo MI, Gallo A, Magliulo G, Fusconi M, et al. Microscopic polyangiitis: advances in diagnostic and therapeutic approaches. Autoimmun Rev. 2015;14:837-44.

83. Mahr A, Belarbi L, Wechsler B, Jeanneret D, Dhote R, Fain O, et al. Population-based prevalence study of Behçet's disease: differences by ethnic origin and low variation by age at immigration. Arthritis Rheum. 2008:58:3951-9.

84. Saadoun D, Wechsler B. Behçet's disease. Orphanet J Rare Dis. 2012;7:20.

85. Hatemi G, Silman A, Bang D, Bodaghi B, Chamberlain AM, Gul A, et al. EULAR recommendations for the management of Behçet disease. Ann Rheum Dis. 2008;67:1656-62.

86. Gilchrist H, Patterson JW. Erythema nodosum and erythema induratum (nodular vasculitis): diagnosis and management. Dermatol Ther. 2010;23:320-7.

87. Mascaró JM, Baselga E. Erythema induratum of Bazin. Dermatol Clin. 2008:26:439-45.

88. Caorsi R, Penco F, Grossi A, Insalaco A, Omenetti A, Alessio M, et al. ADA2 deficiency (DADA2) as an unrecognised cause of early onset polyarteritis nodosa and stroke: a multicentre national study. Ann Rheum Dis. 2017;76:1648-56.

89. De Virgilio A, Greco A, Magliulo G, Gallo A, Ruoppolo G, Conte M, et al. Polyarteritis nodosa: a contemporary overview. Autoimmun Rev. 2016;15:564-70.

90. Hajj-Ali RA, Calabrese LH. Diagnosis and classification of central nervous system vasculitis. J Autoimmun. 2014;48-49:149-52.

91. Salvarani C, Brown RD, Christianson T, Miller DV, Giannini C, Huston J, et al. An Update of the Mayo Clinic Cohort of Patients With Adult Primary Central Nervous System Vasculitis. Medicine (Baltimore) [Internet]. 2015 [cited 2017 Dec 4];94. Available from: https://www.ncbi.nlm.nih. gov/pmc/articles/PMC4616419/

92. Salvarani C, Brown RD, Christianson TJH, Huston J, Giannini C, Miller DV, et al. Adult primary central nervous system vasculitis treatment and course: analysis of one hundred sixty-three patients. Arthritis Rheumatol. 2015;67:1637-45.

93. Calabrese $L H$, Mallek JA. Primary angiitis of the central nervous system. Report of 8 new cases, review of the literature, and proposal for diagnostic criteria. Medicine (Baltimore). 1988;67:20-39.

94. Trapani S, Micheli A, Grisolia F, Resti M, Chiappini E, Falcini F, et al. Henoch Schonlein Purpura in childhood: epidemiological and clinical analysis of 150 cases over a 5-year period and review of literature. Semin Arthritis Rheum. 2005;35:143-53.

95. Ozen S, Pistorio A, lusan SM, Bakkaloglu A, Herlin T, Brik R, et al. EULAR, PRINTO, PRES criteria for Henoch-Schönlein purpura, childhood polyarteritis nodosa, childhood Wegener granulomatosis and childhood Takayasu arteritis: Ankara 2008. Part II: Final classification criteria. Ann Rheum Dis. 2008;2010(69):798-806.

96. Park SJ, Kim HJ, Park H, Hann HJ, Kim KH, Han S, et al. Incidence, prevalence, mortality and causes of death in Takayasu Arteritis in Korea-a nationwide, population-based study. Int J Cardiol. 2017;235:100-4.

97. Watts R, Al-Taiar A, Mooney J, Scott D, MacGregor A. The epidemiology of Takayasu arteritis in the UK. Rheumatology (Oxford). 2009;48:1008-11.

98. Gudbrandsson B, Molberg $\varnothing$, Garen T, Palm $\varnothing$. Prevalence, incidence, and disease characteristics of takayasu arteritis by ethnic background data from a large, population-based cohort resident in southern Norway. Arthritis Care Res. 2017;69:278-85.

99. Birlik M, Kücükyavas Y, Aksu K, Solmaz D, Can G, Taylan A, et al. Epidemiology of Takayasu's arteritis in Turkey. Clin Exp Rheumatol. 2016;34:S33-39.

100. Saruhan-Direskeneli G, Hughes T, Aksu K, Keser G, Coit P, Aydin SZ, et al. Identification of multiple genetic susceptibility loci in Takayasu Arteritis. Am J Hum Genet. 2013;93:298-305.

101. Lutalo PMK, D'Cruz DP. Diagnosis and classification of granulomatosis with polyangiitis (aka Wegener's granulomatosis). J Autoimmun. 2014;48-49:94-8.

102. Yamaguchi M, Ohta A, Tsunematsu T, Kasukawa R, Mizushima Y, Kashiwagi $\mathrm{H}$, et al. Preliminary criteria for classification of adult Still's disease. J Rheumatol. 1992;19:424-30. 
103. Fautrel B, Zing E, Golmard J, Le Moel G, Bissery A, Rioux C, et al. Proposal for a new set of classification criteria for adult-onset still disease. Medicine. 2002;81:194-200.

104. Wynne-Davies R, Hall C, Ansell BM. Spondylo-epiphysial dysplasia tarda with progressive arthropathy. A "new" disorder of autosomal recessive inheritance. Bone Joint J. 1982;64-B:442-5.

105. Wynne-Davies R, Gormley J. The prevalence of skeletal dysplasias. An estimate of their minimum frequency and the number of patients requiring orthopaedic care. Bone Joint J. 1985:67:133-7.

106. Delague V, Chouery E, Corbani S, Ghanem I, Aamar S, Fischer J, et al. Molecular study of WISP3 in nine families originating from the MiddleEast and presenting with progressive pseudorheumatoid dysplasia: identification of two novel mutations, and description of a founder effect. Am J Med Genet A. 2005;138A:118-26.

107. Cassa CA, Smith SE, Docken W, Hoffman E, McLaughlin H, Chun S, et al. An argument for early genomic sequencing in atypical cases: a WISP3 variant leads to diagnosis of progressive pseudorheumatoid arthropathy of childhood. Rheumatology (Oxford). 2016:55:586-9.

108. Nakamura Y, Weidinger G, Liang JO, Aquilina-Beck A, Tamai K, Moon RT, et al. The CCN family member Wisp3, mutant in progressive pseudorheumatoid dysplasia, modulates BMP and Wnt signaling. J Clin Invest. 2007:117:3075-86.

109. Garcia Segarra N, Mittaz L, Campos-Xavier AB, Bartels CF, Tuysuz B, Alanay $Y$, et al. The diagnostic challenge of progressive pseudorheumatoid dysplasia (PPRD): a review of clinical features, radiographic features, and WISP3 mutations in 63 affected individuals. Am J Med Genet. 2012;160C:217-29.

110. Williams CJ, Zhang Y, Timms A, Bonavita G, Caeiro F, Broxholme J, et al. Autosomal dominant familial calcium pyrophosphate dihydrate deposition disease is caused by mutation in the transmembrane protein ANKH. Am J Hum Genet. 2002;71:985-91.

111. Andrew LJ, Brancolini V, de la Pena LS, Devoto M, Caeiro F, Marchegiani $R$, et al. Refinement of the chromosome $5 p$ locus for familial calcium pyrophosphate dihydrate deposition disease. Am J Hum Genet. 1999;64:136-45.

112. Netter P, Bardin T, Bianchi A, Richette P, Loeuille D. The ANKH gene and familial calcium pyrophosphate dihydrate deposition disease. Joint Bone Spine. 2004;71:365-8.

113. Pendleton A, Johnson MD, Hughes A, Gurley KA, Ho AM, Doherty M, et al. Mutations in ANKH cause chondrocalcinosis. Am J Hum Genet. 2002;71:933-40.

114. Rosales-Alexander JL, Balsalobre Aznar J, Magro-Checa C. Calcium pyrophosphate crystal deposition disease: diagnosis and treatment. Open Access Rheumatol. 2014;6:39-47.

115. Verspoor FGM, Zee AAG, Hannink G, van der Geest IC, Veth RPH, et al. Long-term follow-up results of primary and recurrent pigmented villonodular synovitis. Rheumatology (Oxford). 2014;53:2063-70.

116. Myers BW, Masi AT. Pigmented villonodular synovitis and tenosynovitis: a clinical epidemiologic study of 166 cases and literature review. Medicine (Baltimore). 1980;59:223-38.

117. Fiocco U, Sfriso P, Lunardi F, Pagnin E, Oliviero F, Scagliori E, et al. Molecular pathways involved in synovial cell inflammation and tumoral proliferation in diffuse pigmented villonodular synovitis. Autoimmun Rev. 2010;9:780-4.

118. Cassier PA, Italiano A, Gomez-Roca CA, Le Tourneau C, Toulmonde M, Cannarile MA, et al. CSF1R inhibition with emactuzumab in locally advanced diffuse-type tenosynovial giant cell tumours of the soft tissue: a dose-escalation and dose-expansion phase 1 study. Lancet Oncol. 2015;16:949-56.

119. Dwivedi N, Upadhyay J, Neeli I, Khan S, Pattanaik D, Myers L, et al. Felty's syndrome autoantibodies bind to deiminated histones and neutrophil extracellular chromatin traps. Arthritis Rheum. 2012;64:982-92.

120. Hellmich B, Csernok E, Schatz H, Gross WL, Schnabel A. Autoantibodies against granulocyte colony-stimulating factor in Felty's syndrome and neutropenic systemic lupus erythematosus. Arthritis Rheum. 2002:46:2384-91.

121. Balint GP, Balint PV. Felty's syndrome. Best Pract Res Clin Rheumatol. 2004:18:631-45.

122. Narváez J, Domingo-Domenech E, Gómez-Vaquero C, López-Vives L, Estrada P, Aparicio M, et al. Biological agents in the management of felty's syndrome: a systematic review. Semin Arthritis Rheum. 2012;41:658-68.

123. Li H, Altman RD, Yao Q. RS3PE: clinical and research development. Curr Rheumatol Rep. 2015;17:49.

124. Origuchi T, Arima K, Umeda M, Kawashiri S, Tamai M, Nakamura H, et al. Clinical outcomes in the first year of remitting seronegative symmetrical synovitis with pitting edema (RS3PE) syndrome. Mod Rheumatol. 2017;27:150-4.

125. Selmi C, Greenspan A, Huntley A, Gershwin ME. Multicentric ReticuIohistiocytosis: a Critical Review. Current Rheumatology Reports [Internet]. 2015 [cited 2017 Sep 13];17. https://doi.org/10.1007/ s11926-015-0511-6

126. Islam AD, Naguwa SM, Cheema GS, Hunter JC, Gershwin ME. Multicentric reticulohistiocytosis: a rare yet challenging disease. Clin Rev Allergy Immunol. 2013;45:281-9.

127. Brackenridge A, Bashir T, Wheatley T. Multicentric reticulohistiocytosis and pregnancy. BJOG Int J Obst Gynaecol. 2005;112:672-3.

128. Hayem G, Bouchaud-Chabot A, Benali K, Roux S, Palazzo E, SilbermannHoffman O, et al. SAPHO syndrome: a long-term follow-up study of 120 cases. Semin Arthritis Rheum. 1999;29:159-71.

129. Witt M, Meier J, Hammitzsch A, Proft F, Schulze-Koops H, Grunke M. Disease burden, disease manifestations and current treatment regimen of the SAPHO syndrome in Germany: results from a nationwide patient survey. Semin Arthritis Rheum. 2014;43:745-50.

130. Nguyen MT, Borchers A, Selmi C, Naguwa SM, Cheema G, Gershwin ME. The SAPHO syndrome. Semin Arthritis Rheum. 2012;42:254-65.

131. Zimmermann P, Curtis N. Synovitis, acne, pustulosis, hyperostosis, and osteitis (SAPHO) syndrome-a challenging diagnosis not to be missed. J Infect. 2016;72:S106-14.

132. Rukavina I. SAPHO syndrome: a review. J Child Orthop. 2015;9:19-27.

133. Prakken B, Albani S, Martini A. Juvenile idiopathic arthritis. The Lancet. 2011;377:2138-49.

134. Vastert SJ, Kuis W, Grom AA. Systemic JIA: new developments in the understanding of the pathophysiology and therapy. Best Pract Res Clin Rheumatol. 2009:23:655-64.

135. Woo P. Systemic juvenile idiopathic arthritis: diagnosis, management, and outcome. Nat Clin Pract Rheumatol. 2006;2:28-34.

136. Petty RE, Southwood TR, Manners P, Baum J, Glass DN, Goldenberg J, et al. International League of Associations for Rheumatology classification of juvenile idiopathic arthritis: second revision, Edmonton, 2001. J Rheumatol. 2004;31:390-2.

137. Ombrello MJ, Arthur VL, Remmers EF, Hinks A, Tachmazidou I, Grom AA et al. Genetic architecture distinguishes systemic juvenile idiopathic arthritis from other forms of juvenile idiopathic arthritis: clinical and therapeutic implications. Ann Rheum Dis. 2017;76:906-13.

138. Ayaz NA, Özen S, Bilginer Y, Ergüven M, Taşkıran E, Yılmaz E, et al. MEFV mutations in systemic onset juvenile idiopathic arthritis. Rheumatology (Oxford). 2009;48:23-5.

139. De Benedetti F, Meazza C, Vivarelli M, Rossi F, Pistorio A, Lamb R, et al. Functional and prognostic relevance of the -173 polymorphism of the macrophage migration inhibitory factor gene in systemic-onset juvenile idiopathic arthritis. Arthritis Rheum. 2003;48:1398-407.

140. Quartier P, Allantaz F, Cimaz R, Pillet P, Messiaen C, Bardin C, et al. A multicentre, randomised, double-blind, placebo-controlled trial with the interleukin-1 receptor antagonist anakinra in patients with systemic-onset juvenile idiopathic arthritis (ANAJIS trial). Ann Rheum Dis. 2010;70:747-54

141. van Dijkhuizen EHP, Wulffraat NM. Early predictors of prognosis in juvenile idiopathic arthritis: a systematic literature review. Ann Rheum Dis. 2015;74:1996-2005.

142. Edouard S, Fenollar F, Raoult D. The rise of Tropheryma whipplei: a 12-year retrospective study of PCR diagnoses in our reference center. J Clin Microbiol. 2012;50:3917-20.

143. Ramharter M, Harrison N, Bühler T, Herold B, Lagler H, Lötsch F, et al. Prevalence and risk factor assessment of Tropheryma whipplei in a rural community in Gabon: a community-based cross-sectional study. Clin Microbiol Infect. 2014;20:1189-94.

144. Fenollar F, Trani M, Davoust B, Salle B, Birg M, Rolain J, et al. Prevalence of asymptomatic Tropheryma whipplei carriage among humans and nonhuman primates. J Infect Dis. 2008;197:880-7. 
145. Feurle GE, Junga NS, Marth T. Efficacy of ceftriaxone or meropenem as initial Therapies in Whipple's disease. Gastroenterology. 2010;138:478-86.

146. Feurle GE, Moos V, Bläker H, Loddenkemper C, Moter A, Stroux A, et al. Intravenous ceftriaxone, followed by 12 or three months of oral treatment with trimethoprim-sulfamethoxazole in Whipple's disease. J Infect. 2013;66:263-70.

147. Feurle GE, Moos V, Schinnerling K, Geelhaar A, Allers K, Biagi F, et al. The immune reconstitution inflammatory syndrome in whipple disease: a cohort study. Ann Intern Med. 2010;153:710-7.

148. Lagier J-C, Fenollar F, Lepidi H, Giorgi R, Million M, Raoult D. Treatment of classic Whipple's disease: from in vitro results to clinical outcome. J Antimicrob Chemother. 2014;69:219-27.

149. Moos V, Schneider T. Tropheryma whipplei: Erreger verschiedener Infektionen und Pathogen des Morbus Whipple. Dtsch med Wochenschr. 2015; 140:428-32.

150. Pareek A, Sanders TL, Wu IT, Larson DR, Saris DBF, Krych AJ. Incidence of symptomatic osteochondritis dissecans lesions of the knee: a population-based study in Olmsted County. Osteoarthr Cartil. 2017;25:1663-71.

151. Chambers HG, Shea KG, Carey JL. AAOS Clinical Practice Guideline: diagnosis and treatment of osteochondritis dissecans. J Am Acad Orthop Surg. 2011;19:307-9.

152. Kocher MS, Tucker R, Ganley TJ, Flynn JM. Management of Osteochondritis Dissecans of the Knee: Current Concepts Review. Am J Sports Med. 2006;34:1181-91.

153. Aróstegui Jl, Arnal C, Merino R, Modesto C, Antonia Carballo M, Moreno $P$, et al. NOD2 gene-associated pediatric granulomatous arthritis: clinical diversity, novel and recurrent mutations, and evidence of clinical improvement with interleukin-1 blockade in a Spanish cohort. Arthritis Rheum. 2007;56:3805-13.

154. Rosé CD, Aróstegui JI, Martin TM, Espada G, Scalzi L, Yagüe J, et al. NOD2-associated pediatric granulomatous arthritis, an expanding phenotype: study of an international registry and a national cohort in Spain. Arthritis Rheum. 2009;60:1797-803.

155. Caso F, Galozzi P, Costa L, Sfriso P, Cantarini L, Punzi L. Autoinflammatory granulomatous diseases: from Blau syndrome and early-onset sarcoidosis to NOD2-mediated disease and Crohn's disease. RMD Open [Internet]. 2015 [cited 2017 Jun 27];1. Available from: http://www.ncbi. nlm.nih.gov/pmc/articles/PMC4612691/

156. Rosé CD, Pans S, Casteels I, Anton J, Bader-Meunier B, Brissaud P, et al. Blau syndrome: cross-sectional data from a multicentre study of clinical, radiological and functional outcomes. Rheumatology (Oxford). 2015;54:1008-16.

157. Hoffmann AL, Milman N, Byg KE. Childhood sarcoidosis in Denmark 1979-1994: incidence, clinical features and laboratory results at presentation in 48 children. Acta Paediatr. 2004;93:30-6.

158. Mensa-Vilaro A, Cham WT, Tang SP, Lim SC, González-Roca E, Ruiz-Ortiz E, et al. Brief Report: First Identification of Intrafamilial Recurrence of Blau Syndrome due to Gonosomal NOD2 Mosaicism. Arthritis Rheumatol (Hoboken, NJ). 2016;68:1039-44.

159. Finetti M, Omenetti A, Federici S, Caorsi R, Gattorno M. Chronic Infantile Neurological Cutaneous and Articular (CINCA) syndrome: a review. Orphanet J Rare Dis [Internet]. 2016 [cited 2017 Sep 21];11. Available from: http://www.ncbi.nlm.nih.gov/pmc/articles/PMC5142346/

160. Cuisset L, Jeru I, Dumont B, Fabre A, Cochet E, Le Bozec J, et al. Mutations in the autoinflammatory cryopyrin-associated periodic syndrome gene: epidemiological study and lessons from eight years of genetic analysis in France. Ann Rheum Dis. 2011;70:495-9.

161. Kuemmerle-Deschner JB. CAPS—-pathogenesis, presentation and treatment of an autoinflammatory disease. Semin Immunopathol. 2015;37:377-85.

162. Koné-Paut I, Hentgen V, Touitou I. Current data on familial Mediterranean fever. Joint Bone Spine. 2011;78:111-4.

163. Ozen S, Demirkaya E, Amaryan G, Koné-Paut I, Polat A, Woo P, et al. Results from a multicentre international registry of familial Mediterranean fever: impact of environment on the expression of a monogenic disease in children. Ann Rheum Dis. 2014;73:662-7.

164. Sari I, Birlik M, Kasifoglu T. Familial Mediterranean fever: an updated review. Eur J Rheumatol. 2014;1:21-33.
165. Yalçınkaya F, Özen S, Özçakar ZB, Aktay N, Çakar N, Düzova A, et al. A new set of criteria for the diagnosis of familial Mediterranean fever in childhood. Rheumatology (Oxford). 2009;48:395-8.

166. Livneh A, Langevitz P, Zemer D, Zaks N, Kees S, Lidar T, et al. Criteria for the diagnosis of familial mediterranean fever. Arthritis Rheum. 1997:40:1879-85.

167. Haas D, Hoffmann GF. Mevalonate kinase deficiencies: from mevalonic aciduria to hyperimmunoglobulinemia D syndrome. Orphanet J Rare Dis. 2006;1:13.

168. Lainka E, Neudorf U, Lohse P, Timmann C, Bielak M, Stojanov S, et al. Incidence and clinical features of hyperimmunoglobulinemia $D$ and periodic fever syndrome (HIDS) and spectrum of mevalonate kinase (MVK) mutations in German children. Rheumatol Int. 2012;32:3253-60.

169. Ozen S, Kuemmerle-Deschner JB, Cimaz R, Livneh A, Quartier P, KonePaut I, et al. International retrospective chart review of treatment patterns in severe familial mediterranean fever, tumor necrosis factor receptor-associated periodic syndrome, and mevalonate kinase deficiency/hyperimmunoglobulinemia d syndrome: real-world experience in three periodic fever syndromes. Arthritis Care Res. 2017;69:578-86.

170. van der Hilst JCH, Bodar EJ, Barron KS, Frenkel J, Drenth JPH, van der Meer JWM, et al. Long-term follow-up, clinical features, and quality of life in a series of 103 patients with hyperimmunoglobulinemia D syndrome. Medicine. 2008;87:301-10.

171. Arima K, Kinoshita A, Mishima H, Kanazawa N, Kaneko T, Mizushima T, et al. Proteasome assembly defect due to a proteasome subunit beta type 8 (PSMB8) mutation causes the autoinflammatory disorder, Nakajo-Nishimura syndrome. Proc Natl Acad Sci USA. 2011;108:14914-9.

172. McDermott A, Jacks J, Kessler M, Emanuel PD, Gao L. Proteasomeassociated autoinflammatory syndromes: advances in pathogeneses, clinical presentations, diagnosis, and management. Int J Dermatol. 2015;54:121-9.

173. Kanazawa N. Nakajo-Nishimura syndrome: an autoinflammatory disorder showing pernio-like rashes and progressive partial lipodystrophy. Allergol Int. 2012;61:197-206.

174. Schellevis MA, Stoffels M, Hoppenreijs EPAH, Bodar E, Simon A, van der Meer JWM. Variable expression and treatment of PAPA syndrome. Ann Rheum Dis. 2011;70:1168-70.

175. Wise CA, Gillum JD, Seidman CE, Lindor NM, Veile R, Bashiardes S, et al. Mutations in CD2BP1 disrupt binding to PTP PEST and are responsible for PAPA syndrome, an autoinflammatory disorder. Hum Mol Genet. 2002;11:961-9.

176. Lindor NM, Arsenault TM, Solomon H, Seidman CE, McEvoy MT. A new autosomal dominant disorder of pyogenic sterile arthritis, pyoderma gangrenosum, and acne: PAPA syndrome. Mayo Clin Proc. 1997:72:611-5.

177. Yeon HB, Lindor NM, Seidman JG, Seidman CE. Pyogenic arthritis, pyoderma gangrenosum, and acne syndrome maps to chromosome $15 \mathrm{q}$. Am J Hum Genet. 2000;66:1443-8.

178. Førsvoll J, Kristoffersen EK, Øymar K. Incidence, clinical characteristics and outcome in Norwegian children with periodic fever, aphthous stomatitis, pharyngitis and cervical adenitis syndrome; a population-based study. Acta Paediatr. 2013;102:187-92.

179. Thomas KT, Feder HM, Lawton AR, Edwards KM. Periodic fever syndrome in children. J Pediatr. 1999;135:15-21.

180. Vanoni F, Theodoropoulou K, Hofer M. PFAPA syndrome: a review on treatment and outcome. Pediatr Rheumatol Online J [Internet]. 2016 [cited 2017 Oct 12];14. Available from: https://www.ncbi.nlm.nih.gov/ pmc/articles/PMC4924332/

181. Marshall GS, Edwards KM, Butler J, Lawton AR. Syndrome of periodic fever, pharyngitis, and aphthous stomatitis. J Pediatr. 1987;1 10:43-6.

182. Cantarini L, Vitale A, Sicignano LL, Emmi G, Verrecchia E, Patisso I, et al. Diagnostic Criteria for Adult-Onset Periodic Fever, Aphthous Stomatitis, Pharyngitis, and Cervical Adenitis (PFAPA) Syndrome. Front Immunol [Internet]. 2017 [cited 2018 Sep 5];8. Available from: https://www.ncbi. nlm.nih.gov/pmc/articles/PMC5609591/

183. Simon A, Asli B, Braun-Falco M, De Koning H, Fermand J-P, Grattan $C$, et al. Schnitzler's syndrome: diagnosis, treatment, and follow-up. Allergy. 2013;68:562-8.

184. Krause K, Tsianakas A, Wagner N, Fischer J, Weller K, Metz M, et al. Efficacy and safety of canakinumab in Schnitzler syndrome: a multicenter 
randomized placebo-controlled study. J Allergy Clin Immunol. 2017;139:1311-20.

185. Tinazzi E, Puccetti A, Patuzzo G, Sorleto M, Barbieri A, Lunardi C. Schnitzler syndrome, an autoimmune-autoinflammatory syndrome: Report of two new cases and review of the literature. Autoimmun Rev. 2011;10:404-9.

186. Gusdorf L, Asli B, Barbarot S, Néel A, Masseau A, Puéchal X, et al. Schnitzler syndrome: validation and applicability of diagnostic criteria in real-life patients. Allergy. 2017:72:177-82.

187. Vastert SJ, van Wijk R, D'Urbano LE, de Vooght KMK, de Jager W, Ravell $A$, et al. Mutations in the perforin gene can be linked to macrophage activation syndrome in patients with systemic onset juvenile idiopathic arthritis. Rheumatology (Oxford). 2010;49:441-9.

188. Davì S, Minoia F, Pistorio A, Horne A, Consolaro A, Rosina S, et al. Performance of current guidelines for diagnosis of macrophage activation syndrome complicating systemic juvenile idiopathic arthritis. Arthritis Rheumatol. 2014;66:2871-80.

189. Ravelli A, Minoia F, Davì S, Horne A, Bovis F, Pistorio A, et al. Classification criteria for macrophage activation syndrome complicating systemic juvenile idiopathic arthritis. Ann Rheum Dis Ann Rheum Dis. 2016;75:481-9.

190. Henter J-I, Horne A, Aricó M, Egeler RM, Filipovich AH, Imashuku S, et al. HLH-2004: Diagnostic and therapeutic guidelines for hemophagocytic lymphohistiocytosis. Pediatr Blood Cancer. 2007:48:124-31.

191. Minoia F, Davì S, Horne A, Demirkaya E, Bovis F, Li C, et al. Clinical features, treatment, and outcome of macrophage activation syndrome complicating systemic juvenile idiopathic arthritis: a multinational, multicenter study of 362 patients. Arthritis Rheumatol. 2014;66:3160-9.

192. Ruscitti P, Cipriani P, Ciccia F, Masedu F, Liakouli V, Carubbi F, et al. Prognostic factors of macrophage activation syndrome, at the time of diagnosis, in adult patients affected by autoimmune disease: analysis of 41 cases collected in 2 rheumatologic centers. Autoimmun Rev. 2017;16:16-21.

193. Ferguson P, Chen S, Tayeh M, Ochoa L, Leal S, Pelet A, et al. Homozygous mutations in LPIN2 are responsible for the syndrome of chronic recurrent multifocal osteomyelitis and congenital dyserythropoietic anaemia (Majeed syndrome). J Med Genet. 2005;42:551-7.

194. El-Shanti H, Ferguson P. Majeed Syndrome. In: Adam MP, Ardinger HH, Pagon RA, Wallace SE, Bean L, Mefford HC, et al., editors. GeneReviews $\left.{ }^{\circledR}\right)$ [Internet]. Seattle (WA): University of Washington, Seattle; 1993 [cited 2017 Nov 23]. Available from: http://www.ncbi.nlm.nih.gov/ books/NBK1974/

195. Herlin T, Fiirgaard B, Bjerre M, Kerndrup G, Hasle H, Bing X, et al. Efficacy of anti-IL-1 treatment in Majeed syndrome. Ann Rheum Dis. 2013;72:410-3.

196. Lachmann HJ, Papa R, Gerhold K, Obici L, Touitou I, Cantarini L, et al. The phenotype of TNF receptor-associated autoinflammatory syndrome (TRAPS) at presentation: a series of 158 cases from the Eurofever/EUROTRAPS international registry. Ann Rheum Dis. 2014;73:2160-7.

197. Lainka E, Neudorf U, Lohse P, Timmann C, Stojanov S, Huss K, et al. Incidence of TNFRSF1A mutations in German children: epidemiological, clinical and genetic characteristics. Rheumatology. 2009;48:987-91.

198. Bachetti T, Chiesa S, Castagnola P, Bani D, Zanni ED, Omenetti A, et al. Autophagy contributes to inflammation in patients with TNFR-associated periodic syndrome (TRAPS). Ann Rheum Dis. 2013;72:1044-52.

199. Allenbach Y, Drouot L, Rigolet A, Charuel JL, Jouen F, Romero NB, et al. Anti-HMGCR Autoantibodies in European Patients With Autoimmune Necrotizing Myopathies. Medicine (Baltimore) [Internet]. 2014 [cited 2017 Nov 18];93. Available from: https://www.ncbi.nlm.nih.gov/pmc/ articles/PMC4632910/

200. Carroll MB, Newkirk MR, Sumner NS. Necrotizing autoimmune myopathy: a unique subset of idiopathic inflammatory myopathy. J Clin Rheumatol. 2016;22:376-80.

201. Targoff IN. Autoantibodies and their significance in myositis. Curr Rheumatol Rep. 2008;10:333-40.

202. Manole C, Inimioara Mihaela C, Bogdan C. New insights into antisynthetase syndrome. Maedica (Buchar). 2016;11:130-5.

203. Lega J-C, Fabien N, Reynaud Q, Durieu I, Durupt S, Dutertre M, et al. The clinical phenotype associated with myositis-specific and associated autoantibodies: a meta-analysis revisiting the so-called antisynthetase syndrome. Autoimmun Rev. 2014;13:883-91.
204. Cavagna L, Nuño L, Scirè CA, Govoni M, Longo FJL, Franceschini F, et al. Serum Jo-1 autoantibody and isolated arthritis in the antisynthetase syndrome: review of the literature and report of the experience of AENEAS Collaborative Group. Clinic Rev Allerg Immunol. 2017;52:71-80.

205. Lehmann D, Motlagh L, Robaa D, Zierz S. Muscle Carnitine Palmitoyltransferase II Deficiency: A Review of Enzymatic Controversy and Clinical Features. Int J Mol Sci [Internet]. 2017 [cited 2017 Nov 18];18. Available from: https://www.ncbi.nlm.nih.gov/pmc/articles/PMC52 97716/

206. Sigauke E, Rakheja D, Kitson K, Bennett MJ. Carnitine palmitoyltransferase II deficiency: a clinical, biochemical, and molecular review. Lab Invest. 2003;83:1543-54.

207. Ohta A, Nagai M, Nishina M, Tomimitsu H, Kohsaka H. Prevalence and incidence of polymyositis and dermatomyositis in Japan. Mod Rheumatol. 2014;24:477-80.

208. Dobloug C, Garen T, Bitter H, Stjärne J, Stenseth G, Grøvle L, et al. Prevalence and clinical characteristics of adult polymyositis and dermatomyositis; data from a large and unselected Norwegian cohort. Ann Rheum Dis. 2015;74:1551-6.

209. Rosa J, Garrot LF, Navarta DA, Saucedo C, Scolnik M, Bedran Z, et al. Incidence and Prevalence of Polymyositis and Dermatomyositis in a Health Management Organization in Buenos Aires. J Clin Rheumatol. 2013;19:303-7.

210. Malik A, Hayat G, Kalia JS, Guzman MA. Idiopathic Inflammatory Myopathies: Clinical Approach and Management. Front Neurol [Internet]. 2016 [cited 2017 Nov 19];7. Available from: https://www.ncbi.nlm.nih.gov/ pmc/articles/PMC4873503/

211. Dobloug GC, Antal EA, Sveberg L, Garen T, Bitter H, Stjärne J, et al. High prevalence of inclusion body myositis in Norway; a population-based clinical epidemiology study. Eur J Neurol. 2015;22:672-e41.

212. Paltiel AD, Ingvarsson E, Lee DKK, Leff RL, Nowak RJ, Petschke KD, et al. Demographic and clinical features of inclusion body myositis in North America. Muscle Nerve. 2015;52:527-33.

213. Allen JA, Peterson A, Sufit R, Hinchcliff ME, Mahoney JM, Wood TA, et al. Post-epidemic eosinophilia myalgia syndrome associated with L-Tryptophan. Arthritis Rheum [Internet]. 2011 [cited 2017 Nov 9];63. Available from: https://www.ncbi.nlm.nih.gov/pmc/articles/PMC38 48710/

214. Devic P, Gallay L, Streichenberger N, Petiot P. Focal myositis: a review. Neuromuscul Disord. 2016;26:725-33.

215. Auerbach A, Fanburg-smith JC, Wang G, Rushing EJ. Focal myositis: a clinicopathologic study of 115 cases of an intramuscular mass-like reactive process. Am J Surg Pathol. 2009;33:1016-24.

216. De Castro M, Johnston J, Biesecker L. Determining the prevalence of McArdle disease from gene frequency by analysis of next generation sequencing data. Genet Med. 2015;17:1002-6.

217. Santalla A, Nogales-Gadea G, Encinar AB, Vieitez I, González-Quintana A, Serrano-Lorenzo P, et al. Genotypic and phenotypic features of all Spanish patients with McArdle disease: a 2016 update. BMC Genomics. 2017;18:819.

218. Nogales-Gadea G, Santalla A, Brull A, de Luna N, Lucia A, Pinós T. The pathogenomics of McArdle disease-genes, enzymes, models, and therapeutic implications. J Inherit Metab Dis. 2015;38:221-30.

219. Nogales-Gadea G, Pinós T, Andreu AL, Martín MA, Arenas J, Lucia A. Next-generation sequencing to estimate the prevalence of a great unknown: McArdle disease. Genetics in Medicine. 2015;17:gim201576.

220. Musumeci O, Bruno C, Mongini T, Rodolico C, Aguennouz M, Barca $\mathrm{E}$, et al. Clinical features and new molecular findings in muscle phosphofructokinase deficiency (GSD type VII). Neuromuscul Disord. 2012;22:325-30.

221. Toscano A, Musumeci O. Tarui disease and distal glycogenoses: clinical and genetic update. Acta Myol. 2007;26:105-7.

222. Kinoshita A, Saito T, Tomita H, Makita Y, Yoshida K, Ghadami M, et al. Domain-specific mutations in TGFB1 result in Camurati-Engelmann disease. Nat Genet. 2000;26:ng900_19.

223. Simsek-Kiper PO, Dikoglu E, Campos-Xavier B, Utine GE, Bonafe L, Unger $\mathrm{S}$, et al. Positive effects of an angiotensin II type 1 receptor antagonist in Camurati-Engelmann disease: a single case observation. Am J Med Genet. 2014;164:2667-71.

224. Morales-Piga A, Bachiller-Corral J, Trujillo-Tiebas MJ, Villaverde-Hueso A, Gamir-Gamir ML, Alonso-Ferreira V, et al. Fibrodysplasia ossificans 
progressiva in Spain: epidemiological, clinical, and genetic aspects. Bone. 2012;51:748-55.

225. Baujat $G$, Choquet R, Bouée $S$, Jeanbat V, Courouve L, Ruel A, et al. Prevalence of fibrodysplasia ossificans progressiva (FOP) in France: an estimate based on a record linkage of two national databases. Orphanet J Rare Dis [Internet]. 2017 [cited 2017 Nov 17];12. Available from: https://www.ncbi.nlm.nih.gov/pmc/articles/PMC5493013/

226. Heursen E-M, Partida M del CG, Expósito JP, Díaz FN. Osteomesopyknosis - a benign axial hyperostosis that can mimic metastatic disease. Skeletal Radiol. 2016;45:141-6.

227. Meikle PJ, Hopwood JJ, Clague AE, Carey WF. Prevalence of lysosomal storage disorders. JAMA. 1999;281:249-54.

228. Biegstraaten M, Arngrímsson R, Barbey F, Boks L, Cecchi F, Deegan PB, et al. Recommendations for initiation and cessation of enzyme replacement therapy in patients with Fabry disease: the European Fabry Working Group consensus document. Orphanet J Rare Dis. 2015;10:36.

229. Elliott PM, Germain DP, Hilz MJ, Spada M, Wanner C, Falissard B. Why systematic literature reviews in Fabry disease should include all published evidence. European Journal of Medical Genetics. 2019;62:103702.

230. Ehlert K, Frosch M, Fehse N, Zander A, Roth J, Vormoor J. Farber disease: clinical presentation, pathogenesis and a new approach to treatment. Pediatr Rheumatol. 2007;5:15.

231. Alves MQ, Le Trionnaire E, Ribeiro I, Carpentier S, Harzer K, Levade T, et al. Molecular basis of acid ceramidase deficiency in a neonatal form of Farber disease: Identification of the first large deletion in ASAH1 gene. Mol Genet Metab. 2013;109:276-81.

232. Orenstein M, Barbouth D, Bodamer OA, Weinreb NJ. Patients with type 1 Gaucher disease in South Florida, USA: demographics, genotypes, disease severity and treatment outcomes. Orphanet J Rare Dis. 2014;9:45.

233. Stirnemann J, Vigan M, Hamroun D, Heraoui D, Rossi-Semerano L, Berger MG, et al. The French Gaucher's disease registry: clinical characteristics, complications and treatment of 562 patients. Orphanet J Rare Dis. 2012;7:77.

234. Stirnemann J, Belmatoug N, Camou F, Serratrice C, Froissart R, Caillaud C, et al. A Review of Gaucher Disease Pathophysiology, Clinical Presentation and Treatments. Int J Mol Sci [Internet]. 2017 [cited 2017 Nov 24];18. Available from: https://www.ncbi.nlm.nih.gov/pmc/articles/ PMC5343975/

235. Nalysnyk L, Rotella P, Simeone JC, Hamed A, Weinreb N. Gaucher disease epidemiology and natural history: a comprehensive review of the literature. Hematology. 2017;22:65-73.

236. Gurevich E, Hershkovitz E, Yarza S, Landau D. High prevalence of hypophosphatasia in Southern Israel. Acta Paediatr. 2020;109:851-2.

237. Högler W, Langman C, Gomes da Silva H, Fang S, Linglart A, Ozono K, et al. Diagnostic delay is common among patients with hypophosphatasia: initial findings from a longitudinal, prospective, global registry. BMC Musculoskelet Disord [Internet]. 2019 [cited 2020 Sep 7];20. Available from: https://www.ncbi.nlm.nih.gov/pmc/articles/PMC6376686/

238. Mornet E, Yvard A, Taillandier A, Fauvert D, Simon-Bouy B. A MolecularBased Estimation of the Prevalence of Hypophosphatasia in the European Population. Ann Hum Genet. 2011;75:439-45.

239. Montaño AM, Tomatsu S, Gottesman GS, Smith M, Orii T. International Morquio A Registry: clinical manifestation and natural course of Morquio A disease. J Inherit Metab Dis. 2007;30:165-74.

240. Leadley RM, Lang S, Misso K, Bekkering T, Ross J, Akiyama T, et al. A systematic review of the prevalence of Morquio A syndrome: challenges for study reporting in rare diseases. Orphanet J Rare Dis [Internet]. 2014 [cited 2017 Nov 24];9. Available from: https://www. ncbi.nlm.nih.gov/pmc/articles/PMC4251694/

241. Hendriksz CJ, Berger Kl, Giugliani R, Harmatz P, Kampmann C, Mackenzie WG, et al. International guidelines for the management and treatment of morquio a syndrome. Am J Med Genet A. 2015;167:11-25.

242. Kotwal A, Clarke BL. Melorheostosis: a rare sclerosing bone dysplasia. Curr Osteoporos Rep. 2017;15:335-42.

243. Smith GC, Pingree MJ, Freeman LA, Matsumoto JM, Howe BM, Kannas SN, et al. Melorheostosis: a retrospective clinical analysis of 24 patients at the Mayo Clinic. PM\&R. 2017;9:283-8.

244. Freyschmidt J. Melorheostosis: a review of 23 cases. Eur Radiol. 2001;11:474-9.
245. Busch J, Frank V, Bachmann N, Otsuka A, Oji V, Metze D, et al. Mutations in the prostaglandin transporter SLCO2A1 cause primary hypertrophic osteoarthropathy with digital clubbing. J Investig Dermatol. 2012;132:2473-6.

246. Lee S, Park SY, Kwon HJ, Lee C-H, Kim O-H, Rhee Y. Identification of the mutations in the prostaglandin transporter gene, SLCO2A1 and clinical characterization in korean patients with pachydermoperiostosis. J Korean Med Sci. 2016;31:735-42.

247. Uppal S, Diggle CP, Carr IM, Fishwick CWG, Ahmed M, Ibrahim GH, et al. Mutations in 15-hydroxyprostaglandin dehydrogenase cause primary hypertrophic osteoarthropathy. Nat Genet. 2008:40:789-93.

248. Jurecka A, Ługowska A, Golda A, Czartoryska B, Tylki-Szymańska A. Prevalence rates of mucopolysaccharidoses in Poland. J Appl Genet. 2015;56:205-10.

249. Malm G, Lund AM, Månsson J-E, Heiberg A. Mucopolysaccharidoses in the Scandinavian countries: incidence and prevalence. Acta Pædiatrica. 2008;97:1577-81.

250. Guffon N, Heron B, Chabrol B, Feillet F, Montauban V, Valayannopoulos $V$. Diagnosis, quality of life, and treatment of patients with Hunter syndrome in the French healthcare system: a retrospective observational study. Orphanet J Rare Dis [Internet]. 2015 [cited 2017 Nov 24];10. Available from: https://www.ncbi.nlm.nih.gov/pmc/artic les/PMC4407793/

251. Kitaoka T, Miyoshi Y, Namba N, Miura K, Kubota T, Ohata Y, et al. Two Japanese familial cases of Caffey disease with and without the common COL1A1 mutation and normal bone density, and review of the literature. Eur J Pediatr. 2014;173:799-804.

252. Gensure RC, Mäkitie O, Barclay C, Chan C, DePalma SR, Bastepe M, et al. A novel COL1A1 mutation in infantile cortical hyperostosis (Caffey disease) expands the spectrum of collagen-related disorders. J Clin Invest. 2005:115:1250-7.

253. Guerin A, Dupuis L, Mendoza-Londono R. Caffey Disease. In: Adam MP, Ardinger HH, Pagon RA, Wallace SE, Bean LJ, Mefford HC, et al., editors. GeneReviews ${ }^{\circledR}$ [Internet]. Seattle (WA): University of Washington, Seattle; 1993 [cited 2017 Dec 5]. Available from: http://www.ncbi.nlm.nih. gov/books/NBK99168/

254. Germain DP. Ehlers-Danlos syndrome type IV. Orphanet J Rare Dis. 2007;2:32.

255. De Paepe A, Malfait F. The Ehlers-Danlos syndrome, a disorder with many faces. Clin Genet. 2012;82:1-11.

256. Malfait F, Francomano C, Byers P, Belmont J, Berglund B, Black J, et al. The 2017 international classification of the Ehlers-Danlos syndromes. Am J Med Genet. 2017;175:8-26.

257. Gorospe L, Ayala-Carbonero AM, Fernández-Méndez MÁ, Arrieta P, Muñoz-Molina GM, Cabañero-Sánchez A, et al. Idiopathic fibrosing mediastinitis: spectrum of imaging findings with emphasis on its association with IgG4-related disease. Clin Imaging. 2015;39:993-9.

258. Rossi SE, McAdams HP, Rosado-de-Christenson ML, Franks TJ, Galvin JR. Fibrosing mediastinitis. Radiographics. 2001;21:737-57.

259. Wu Z, Jarvis H, Howard LS, Wright C, Kon OM. Post-tuberculous fibrosing mediastinitis: a review of the literature. BMJ Open Respir Res [Internet]. 2017 [cited 2017 Dec 8];4. Available from: https://www.ncbi. nIm.nih.gov/pmc/articles/PMC5501238/

260. Uchida K, Masamune A, Shimosegawa T, Okazaki K. Prevalence of IgG4-Related Disease in Japan Based on Nationwide Survey in 2009. Int J Rheumatol [Internet]. 2012 [cited 2020 Sep 7];2012. Available from: https://www.ncbi.nlm.nih.gov/pmc/articles/PMC3415093/

261. Umehara H, Okazaki K, Masaki Y, Kawano M, Yamamoto M, Saeki T, et al. A novel clinical entity, IgG4-related disease (IgG4RD): general concept and details. Mod Rheumatol. 2012;22:1-14.

262. Wallace ZS, Zhang Y, Perugino CA, Naden R, Choi HK, Stone JH. Clinical phenotypes of IgG4-related disease: an analysis of two international cross-sectional cohorts. Ann Rheum Dis. 2019;78:406-12.

263. Wallace ZS, Naden RP, Chari S, Choi H, Della-Torre E, Dicaire J-F, et al. The 2019 American College of Rheumatology/European League Against Rheumatism Classification Criteria for IgG4-Related Disease. Arthritis Rheumatol (Hoboken, NJ). 2020;72:7-19.

264. Groth KA, Hove H, Kyhl K, Folkestad L, Gaustadnes M, Vejlstrup N, et al. Prevalence, incidence, and age at diagnosis in Marfan Syndrome. Orphanet J Rare Dis [Internet]. 2015 [cited 2017 Nov 23];10. Available from: https://www.ncbi.nlm.nih.gov/pmc/articles/PMC4668669/ 
265. Loeys BL, Dietz HC, Braverman AC, Callewaert BL, Backer JD, Devereux $\mathrm{RB}$, et al. The revised Ghent nosology for the Marfan syndrome. J Med Genet. 2010;47:476-85.

266. Judge DP, Dietz HC. Marfan's syndrome. Lancet. 2005;366:1965-76.

267. Pinal-Fernandez I, Selva-O'Callaghan A, Grau JM. Diagnosis and classification of eosinophilic fasciitis. Autoimmun Rev. 2014;13:379-82.

268. Mazori DR, Femia AN, Vleugels RA. Eosinophilic fasciitis: an updated review on diagnosis and treatment. Curr Rheumatol Rep. 2017;19:74.

269. Gunnarsson R, Molberg Ø, Gilboe I-M, Gran JT, Group PS. The prevalence and incidence of mixed connective tissue disease: a national multicentre survey of Norwegian patients. Ann Rheum Dis. 2011;70:1047-51.

270. Tani C, Carli L, Vagnani S, Talarico R, Baldini C, Mosca M, et al. The diagnosis and classification of mixed connective tissue disease. J Autoimmun. 2014;48-49:46-9.

271. Amigues JM, Cantagrel A, Abbal M, Mazieres B. Comparative study of 4 diagnosis criteria sets for mixed connective tissue disease in patients with anti-RNP antibodies. Autoimmunity Group of the Hospitals of Toulouse. J Rheumatol. 1996;23:2055-62.

272. Valter I, Saretok S, Marica HR. Prevalence of scleroderma spectrum disorders in the general population of Estonia. Scand J Rheumatol. 1997;26:419-25

273. Ferri C, Valentini G, Cozzi F, Sebastiani M, Michelassi C, La Montagna G, et al. Systemic sclerosis: demographic, clinical, and serologic features and survival in 1,012 Italian Patients. Medicine. 2002;81:139.

274. van den Hoogen F, Khanna D, Fransen J, Johnson SR, Baron M, Tyndall A, et al. 2013 classification criteria for systemic sclerosis: an American college of rheumatology/European league against rheumatism collaborative initiative. Ann Rheum Dis. 2013;72:1747-55.

275. Mayes MD, Lacey JV, Beebe-Dimmer J, Gillespie BW, Cooper B, Laing $\mathrm{TJ}$, et al. Prevalence, incidence, survival, and disease characteristics of systemic sclerosis in a large US population. Arthritis Rheum. 2003:48:2246-55

276. Martinez-Lopez A, Blasco-Morente G, Perez-Lopez I, Herrera-Garcia J, Luque-Valenzuela M, Sanchez-Cano D, et al. CLOVES syndrome: review of a PIK3CA-related overgrowth spectrum (PROS). Clin Genet. 2017:91:14-21.

277. Keppler-Noreuil KM, Rios JJ, Parker VER, Semple RK, Lindhurst MJ, Sapp JC, et al. PIK3CA-related overgrowth spectrum (PROS): diagnostic and testing eligibility criteria differential diagnosis and evaluation. Am J Med Genet A. 2015;1:287-95.

278. Kurek KC, Luks VL, Ayturk UM, Alomari Al, Fishman SJ, Spencer SA, et al. Somatic mosaic activating mutations in PIK3CA cause CLOVES syndrome. Am J Hum Genet. 2012;90:1108-15.

279. Keppler-Noreuil KM, Sapp JC, Lindhurst MJ, Parker VE, Blumhorst C, Darling $T$, et al. Clinical delineation and natural history of the PIK3CArelated overgrowth spectrum. Am J Med Genet A. 2014;164:1713-33.

280. Kihiczak GG, Meine JG, Schwartz RA, Janniger CK. Klippel-Trenaunay syndrome: a multisystem disorder possibly resulting from a pathogenic gene for vascular and tissue overgrowth. Int J Dermatol. 2006;45:883-90.

281. Wang SK, Drucker NA, Gupta AK, Marshalleck FE, Dalsing MC. Diagnosis and management of the venous malformations of Klippel-Trénaunay syndrome. J Vasc Surg Venous Lymphat Disord. 2017;5:587-95.

282. Dimopoulos A, Sicko RJ, Kay DM, Rigler SL, Fan R, Romitti PA, et al. Copy number variants in a population-based investigation of Klippel-Trenaunay syndrome. Am J Med Genet. 2017;173:352-9.

283. Zhang T, Yao Y, Wang J, Li Y, He P, Pasupuleti V, et al. Haploinsufficiency of Klippel-Trenaunay syndrome gene Aggf1 inhibits developmental and pathological angiogenesis by inactivating PI3K and AKT and disrupts vascular integrity by activating VE-cadherin. Hum Mol Genet. 2016:25:5094-110.

284. Lindhurst MJ, Sapp JC, Teer JK, Johnston JJ, Finn EM, Peters K, et al. A mosaic activating mutation in AKT1 associated with the proteus syndrome. N Engl J Med. 2011;365:611-9.

285. Cohen MM. Proteus syndrome review: molecular, clinical, and pathologic features. Clin Genet. 2014;85:111-9.

286. Turner JT, Cohen MM, Biesecker LG. Reassessment of the proteus syndrome literature: application of diagnostic criteria to published cases. Am J Med Genet. 2004;130A:111-22.
287. Cives M, Simone V, Rizzo FM, Dicuonzo F, Cristallo Lacalamita M, Ingravallo G, et al. Erdheim-Chester disease: a systematic review. Crit Rev Oncol Hematol. 2015;95:1-11.

288. Diamond EL, Dagna L, Hyman DM, Cavalli G, Janku F, Estrada-Veras J, et al. Consensus guidelines for the diagnosis and clinical management of Erdheim-Chester disease. Blood. 2014;124:483-92.

289. Cavalli G, Guglielmi B, Berti A, Campochiaro C, Sabbadini MG, Dagna L. The multifaceted clinical presentations and manifestations of ErdheimChester disease: comprehensive review of the literature and of 10 new cases. Ann Rheum Dis. 2013;72:1691-5.

290. Mazor RD, Manevich-Mazor M, Shoenfeld Y. Erdheim-Chester disease: a comprehensive review of the literature. Orphanet J Rare Dis. 2013;8:137.

291. Hanks S, Adams S, Douglas J, Arbour L, Atherton DJ, Balci S, et al. Mutations in the gene encoding capillary morphogenesis protein 2 cause juvenile hyaline fibromatosis and infantile systemic hyalinosis. Am J Hum Genet. 2003;73:791-800.

292. Haidar Z, Temanni R, Chouery E, Jitesh P, Liu W, Al-Ali R, et al. Diagnosis implications of the whole genome sequencing in a large Lebanese family with hyaline fibromatosis syndrome. BMC Genet [Internet]. 2017 [cited 2017 Nov 21];18. Available from: https://www.ncbi.nlm.nih.gov/ pmc/articles/PMC5244738/

293. Shieh JTC, Swidler P, Martignetti JA, Ramirez MCM, Balboni I, Kaplan J, et al. Systemic hyalinosis: a distinctive early childhood-onset disorder characterized by mutations in the anthrax toxin receptor 2 gene (ANTRX2). Pediatrics. 2006;118:e1485-92.

294. Lindvall LE, Kormeili T, Chen E, Ramirez MCM, Grum-Tokars V, Glucksman $M J$, et al. Infantile systemic hyalinosis: case report and review of the literature. J Am Acad Dermatol. 2008;58:303-7.

295. Marzano AV, Ishak RS, Saibeni S, Crosti C, Meroni PL, Cugno M. Autoinflammatory skin disorders in inflammatory bowel diseases, pyoderma gangrenosum and sweet's syndrome: a comprehensive review and disease classification criteria. Clinic Rev Allerg Immunol. 2013;45:202-10.

296. Ghoufi L, Ortonne N, Ingen-Housz-Oro S, Barhoumi W, Begon E, Haioun C, et al. Histiocytoid Sweet Syndrome Is More Frequently Associated With Myelodysplastic Syndromes Than the Classical Neutrophilic Variant. Medicine (Baltimore) [Internet]. 2016 [cited 2017 Oct 18];95. Available from: https://www.ncbi.nlm.nih.gov/pmc/articles/PMC4839791/

297. Cohen PR. Sweet's syndrome-a comprehensive review of an acute febrile neutrophilic dermatosis. Orphanet J Rare Dis. 2007;2:34.

298. Su WP, Liu HN. Diagnostic criteria for Sweet's syndrome. Cutis. 1986;37:167-74.

299. Walker DC, Cohen PR. Trimethoprim-sulfamethoxazole-associated acute febrile neutrophilic dermatosis: Case report and review of druginduced Sweet's syndrome. J Am Acad Dermatol. 1996:34:918-23.

300. von den Driesch P. Sweet's syndrome (acute febrile neutrophilic dermatosis). J Am Acad Dermatol. 1994:31:535-56.

301. Horváth A, Páll N, Molnár K, Kováts T, Surján G, Vicsek T, et al. A nationwide study of the epidemiology of relapsing polychondritis. Clin Epidemiol. 2016;8:211-30.

302. Dion J, Costedoat-Chalumeau N, Sène D, Cohen-Bittan J, Leroux G, Dion C, et al. Relapsing polychondritis can be characterized by three different clinical phenotypes: analysis of a recent series of 142 patients. Arthritis Rheumatol. 2016:68:2992-3001.

303. Mathew SD, Battafarano DF, Morris MJ. Relapsing polychondritis in the department of defense population and review of the literature. Semin Arthritis Rheum. 2012;42:70-83.

304. Michet CJ. Relapsing polychondritis: survival and predictive role of early disease manifestations. Ann Intern Med. 1986;104:74.

305. McAdam LP, O'Hanlan MA, Bluestone R, Pearson CM. Relapsing polychondritis: prospective study of 23 patients and a review of the literature. Medicine (Baltimore). 1976;55:193-215.

306. Damiani JM, Levine HL. Relapsing polychondritis-report of ten cases. Laryngoscope. 1979;89:929-46.

307. Durtette C, Hachulla E, Resche-Rigon M, Papo T, Zénone T, Lioger B, et al. Cogan syndrome: characteristics, outcome and treatment in a French nationwide retrospective study and literature review. Autoimmun Rev. 2017;16:1219-23.

308. Gluth MB, Baratz KH, Matteson EL, Driscoll CLW. Cogan syndrome: a retrospective review of 60 patients throughout a half century. Mayo Clinic Proc. 2006;81:483-8. 
309. Tayer-Shifman OE, Ilan O, Tovi H, Tal Y. Cogan's syndrome-clinical guidelines and novel therapeutic approaches. Clinic Rev Allerg Immunol. 2014;47:65-72.

310. Wang Y, Zhao J, Ji L lan, Zhang S, Zhang Z. Weber-Christian disease present with lung nodules dramatically improved with corticosteroid therapy: one case report and literature review. Int J Rheum Dis. 2015;n/a-n/a.

311. Verrilli S, Ciarnella A, Laganà B, Calafiore S, Guglielmelli F, Basile L, et al. Ocular inflammation: can it be a sign of activity of Weber-Christian disease? A case report and review of literature. Ocul Immunol Inflamm. 2016:24:223-6.

312. White JW, Winkelmann RK. Weber-Christian panniculitis: a review of 30 cases with this diagnosis. J Am Acad Dermatol. 1998;39:56-62.

313. Mavrikakis I, Georgiadis T, Fragiadaki K, Sfikakis PP. Orbital lobular panniculitis in Weber-Christian disease: sustained response to anti-TNF treatment and review of the literature. Surv Ophthalmol. 2010;55:584-9.

314. Cohen SS, Skovbo S, Vestergaard H, Kristensen T, Møller M, Bindslev-

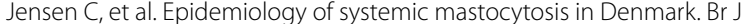
Haematol. 2014;166:521-8.

315. Lim K-H, Tefferi A, Lasho TL, Finke C, Patnaik M, Butterfield JH, et al. Systemic mastocytosis in 342 consecutive adults: survival studies and prognostic factors. Blood. 2009;113:5727-36
316. Pardanani A. Systemic mastocytosis in adults: 2017 update on diagnosis, risk stratification and management. Am J Hematol. 2016;91:1146-59.

317. Pieri L, Bonadonna P, Elena C, Papayannidis C, Grifoni FI, Rondoni $M$, et al. Clinical presentation and management practice of systemic mastocytosis. A survey on 460 Italian patients. Am J Hematol. 2016;91:692-9.

318. Valent P, Akin C, Metcalfe DD. Mastocytosis: 2016 updated WHO classification and novel emerging treatment concepts. Blood. 2017; 129:1420-7.

319. Dubrey S, Shah S, Hardman T, Sharma R. Sarcoidosis: the links between epidemiology and aetiology. Postgrad Med J. 2014;90:582-9.

320. Nicholson TT, Plant BJ, Henry MT. Sarcoidosis in Ireland: regional differences in prevalence and mortality from 1996-2005. Sarcoidosis Vasc Diffuse Lung Dis. 2010;27:111-20.

321. WessendorfTE, Bonella F, Costabel U. Diagnosis of sarcoidosis. Clinic Rev Allerg Immunol. 2015;49:54-62.

\section{Publisher's Note}

Springer Nature remains neutral with regard to jurisdictional claims in published maps and institutional affiliations.
Ready to submit your research? Choose BMC and benefit from:

- fast, convenient online submission

- thorough peer review by experienced researchers in your field

- rapid publication on acceptance

- support for research data, including large and complex data types

- gold Open Access which fosters wider collaboration and increased citations

- maximum visibility for your research: over $100 \mathrm{M}$ website views per year

At BMC, research is always in progress.

Learn more biomedcentral.com/submissions 